\title{
Ontology for Life-Cycle Modeling of Water Distribution Systems: Application of Model View Definition Attributes
}

Kristine Fallon Associates, Inc.

11 E. Adams Street, Suite 1100

Chicago, IL 60603

Prepared under CRADA-07-CERL-02 under the supervision of

E. William East, Project Manager (CEERD-CF-N)

Construction Engineering Research Laboratory

US Army Engineer Research and Development Center 2902 Newmark Drive

Champaign, IL 61822 
The US Army Engineer Research and Development Center (ERDC) solves the nation's toughest engineering and environmental challenges. ERDC develops innovative solutions in civil and military engineering, geospatial sciences, water resources, and environmental sciences for the Army, the Department of Defense, civilian agencies, and our nation's public good. Find out more at www.erdc.usace.army.mil.

To search for other technical reports published by ERDC, visit the ERDC online library at http://acwc.sdp.sirsi.net/client/default. 


\section{Ontology for Life-Cycle Modeling of Water Distribution Systems: Application of Model View Definition Attributes}

Kristine K. Fallon, Robert A. Feldman, Gregory Williams, and Omobolawa Fadojutimi

Kristine Fallon Associates, Inc.

11 E. Adams Street, Suite 1100

Chicago, IL 60603

Tim Chipman

Constructivity.com, LLC

2625 North Loop Drive

Ames, lowa 50010

Final report

Approved for public release; distribution is unlimited.

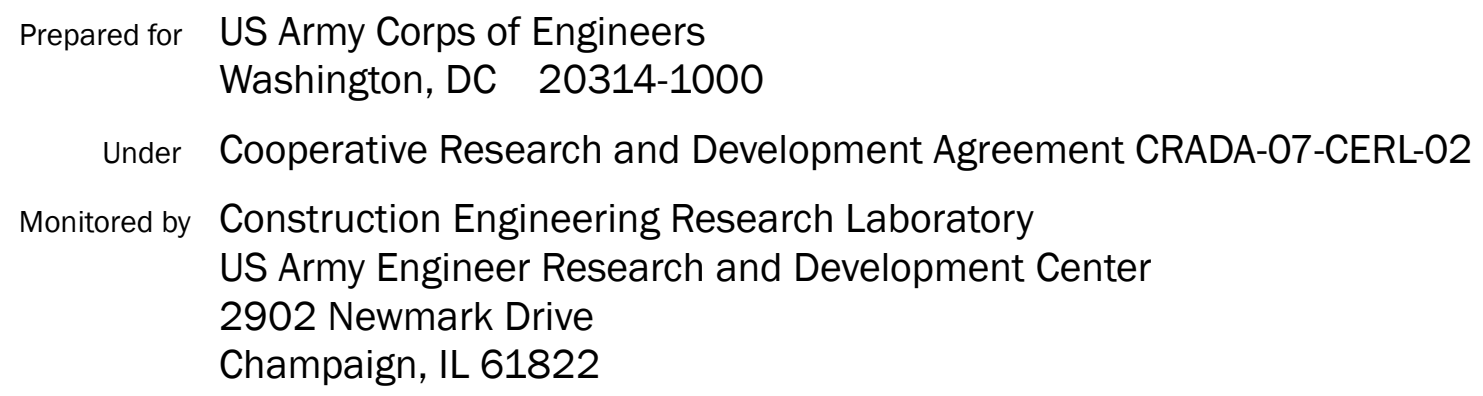




\section{Abstract}

Previous efforts by the US Army Engineer Research and Development Center, Construction Engineering Research Laboratory (ERDC-CERL) to develop a life-cycle building model have resulted in the definition of a "core" building information model that contains general information describing facility assets such as spaces and equipment. To describe how facility assets (i.e., components) function together, information about assemblies of assets and their connections must also be defined. The definitions of assets, assemblies, and connections for the various buildinginformation domains are discipline-specific. Work documented in ERDC/CERL CR-13-4 identified the processes and tasks specifically associated with the design of interior plumbing systems and the information exchange requirements for every participant in the design. The findings were used to develop an information-exchange Model View Definition (MVD) for building water systems.

The objective of the current work was to document the steps needed to identify the plumbing MVD attributes in three experimental building information models representing typical low-rise Army facilities, and to update those models. This work also validated the International Foundation Class (IFC) export function from the experimental models against the water system MVD, and studied the requirements for creating computable open building models that can be utilized for the automated information exchanges.

DISCLAIMER: The contents of this report are not to be used for advertising, publication, or promotional purposes. Citation of trade names does not constitute an official endorsement or approval of the use of such commercial products. All product names and trademarks cited are the property of their respective owners. The findings of this report are not to be construed as an official Department of the Army position unless so designated by other authorized documents. 


\section{Contents}

Abstract..................................................................................................................

List of Figures and Tables ................................................................................................

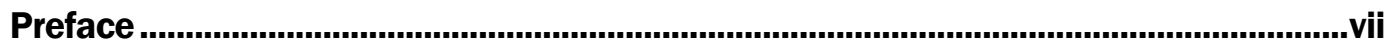

Unit Conversion Factors................................................................................................tii

1 Introduction ....................................................................................................... 1

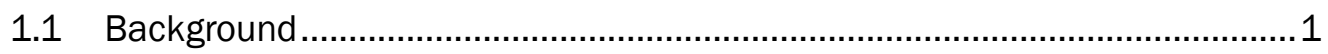

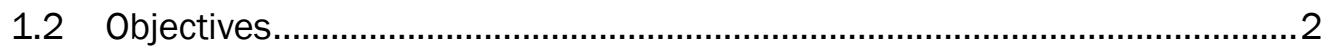

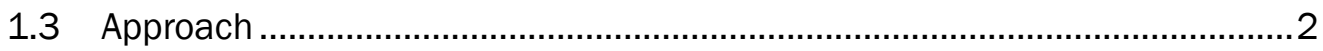

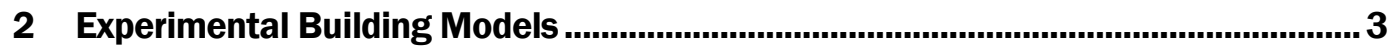

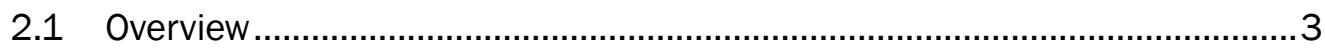

2.2 Determination of additional water system properties.................................. 4

2.3 Addition of water system properties to the Revit experimental

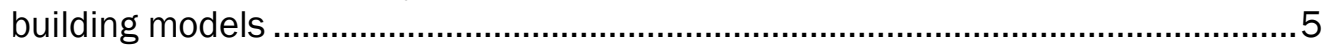

2.3.1 Removing nonessential elements from the models ........................................... 5

2.3.2 Modeling additional water system elements............................................................ 5

2.3.3 Adding properties to elements in the models .................................................... 6

2.3.4 Assigning default values.................................................................................... 7

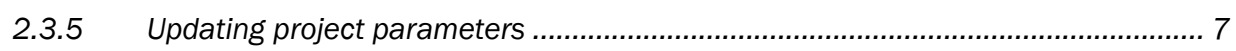

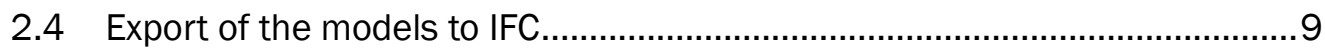

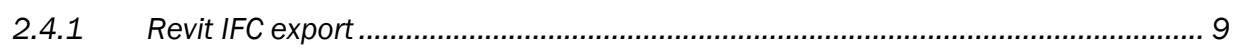

2.4.2 Special considerations regarding Revit IFC export ............................................... 10

2.5 Validation of the Revit 2013 IFC export ................................................ 10

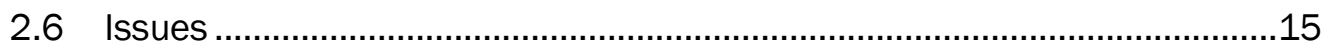

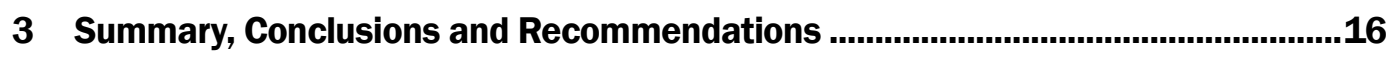

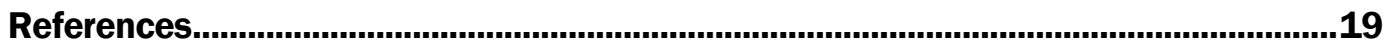

Appendix A: Water System Building Component Occurrences in Revit Models...........20

Appendix B: Additional Water System Properties for Revit Families and Model .........22

Appendix C: Sample Validation Report .................................................................................. 30

Report Documentation Page 


\section{Figures and Tables}

\section{Figures}

Figure 1. Revit parameter properties dialog box showing the lamp efficiency property applied to lighting fixtures.

Figure 2. Revit Parameter Properties dialog box showing the building ID property applied to Project Information 8

Figure 3. Revit Project Properties dialog box showing the building parameters.

\section{Tables}

Table 1. Model statistics. ...................................................................................................... 4

Table 2. IFC export override parameter settings. .............................................................. 9

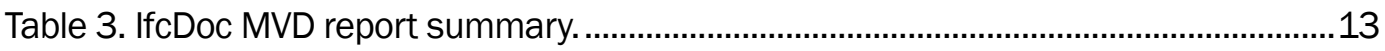

Table 4. IfcDoc MVD non-compliance items. ……................................................................. 


\section{Preface}

This study was conducted for the US Army Engineer Research and Development Center, Construction Engineering Research Laboratory (ERDCCERL) and the National Institute of Building Sciences (NIBS) by Kristine Fallon Associates Inc., and Constructivity.com, LLC, under CRADA-07CERL-02, "Cooperative Research and Development Agreement Between US Army Engineer Research and Development Center- Construction Engineering Laboratory and National Institute Of Building Sciences." The CRADA supports Research, Development, Test, and Evaluation (RDT\&E) Program Element 622784 T41, "Military Facilities Engineering Technology"; Project 157249, "Life-Cycle Model For Mission Ready Sustainable Facilities (LCM)." The ERDC-CERL project manager was Dr. E. William East (CEERD-CF-N), and the NIBS project manager was Dominique Fernandez.

The work was supervised and monitored by the Engineering Processes Branch (CF-N) of the Facilities Division (CF), ERDC-CERL. At the time of publication, Donald K. Hicks was Chief, CEERD-CF-N; L. Michael Golish was Chief, CEERD-CF; and Martin J. Savoie, CEERD-CV-ZT, was the Technical Director for Installations. The Deputy Director of ERDC-CERL was Dr. Kirankumar Topudurti and the Director was Dr. Ilker Adiguzel.

COL Kevin J. Wilson was the Commander of ERDC, and Dr. J effery P. Holland was the Director. 


\section{Unit Conversion Factors}

\begin{tabular}{|l|c|l|}
\hline Multiply & By & To Obtain \\
\hline cubic inches & $1.6387064 \mathrm{E}-05$ & cubic meters \\
\hline cubic yards & 0.7645549 & cubic meters \\
\hline degrees (angle) & 0.01745329 & radians \\
\hline feet & 0.3048 & meters \\
\hline gallons (U.S. liquid) & $3.785412 \mathrm{E}-03$ & cubic meters \\
\hline miles (U.S. statute) & $1,609.347$ & meters \\
\hline mils & 0.0254 & millimeters \\
\hline square feet & 0.09290304 & square meters \\
\hline square inches & $6.4516 \mathrm{E}-04$ & square meters \\
\hline square miles & $2.589998 \mathrm{E}+06$ & square meters \\
\hline square yards & 0.8361274 & square meters \\
\hline yards & 0.9144 & meters \\
\hline
\end{tabular}




\section{Introduction}

\subsection{Background}

Previous efforts by the US Army Engineer Research and Development Center, Construction Engineering Research Laboratory (ERDC-CERL) to develop a life-cycle building model have resulted in the definition of a "core" building information model that contains general information describing facility assets such as spaces and equipment (East, Love, and Nisbet 2010). To describe how facility assets (i.e., components) function together, information about assemblies of assets and their connections must also be defined. The definitions of assets, assemblies, and connections for the various building-information domains are discipline-specific. Taken together, studies of all essential building-information domains will create a unified framework for developing automatic design checks, ensuring construction compliance, improving operations and maintenance efficiency, and evaluating alternatives for redesign within completed facilities.

COBie (East 2007) was the first step in analyzing information exchanges in the life cycle of a building. Since March 2012, COBie has been part of the National BIM Standard- United States (NBIMS-US). COBie defines the format for providing information about building assets from the planning phase through design, construction, and operations. Properties of these assets may also be captured in the COBie date exchange format. The COBie Guide, a commentary on the COBie standard (public draft downloadable at link from http://www.nibs.org/?page=bsa_cobieguide), does not prescribe how to model specific assemblies of components or how components and assemblies are connected. Those aspects of modeling and information exchange require a domain-specific ontology for every system needed to construct a functional building. Subsequent work led to the development of a Model View Design (MVD) for the design of water distribution systems in typical Army facilities. That MVD is documented in ERDC/ CERL CR-13-4 (Chipman et al. 2013).

In order to provide a foundation for testing and validating research on building life-cycle topics, ERDC-CERL led the development of experimental core building information models for three common facility types widely used on Army installations: a Duplex Apartment, a Medical Clinic, and an Office Building (East 2012a). The present report documents the 
application of the water distribution system MVD to those experimental models as part of the effort to create formal specifications applicable to open-standards BIM at the coordinated design stage of construction.

\subsection{Objectives}

The main objective of this work was to document the steps needed to identify the water system MVD attributes in the three experimental BIMs and update the models. This work also validated the International Foundation Class (IFC) export function from the experimental models against the MVD, and studied the requirements for creating computable open building models that can be utilized for the automated information exchanges.

\subsection{Approach}

In this report, the term water distribution systems encompasses the plumbing supply and waste removal systems for flow delivery terminals such as sinks, toilets, showers and bath tubs. The present work applies the process flows and data exchanges requirements for the design of interior water distribution systems in typical Army facilities. These processes and exchange requirements were used to create a Model View Definition (MVD) procedures (see Chipman et al. 2013) specified by the International Organization for Standardization (ISO) and buildingSmart International (e.g., Hietanen 2008).

The water system process flow and exchange requirements were applied to a simulated real-world situation to study how the information exchanges can be handled in COTS BIM software using the three experimental models noted above. These "core" BIMs represent typical low-rise facilities commonly built on Army installations. Water distribution equipment and systems were updated using commercial off-the-shelf (COTS) BIM software and a native common object library developed in that software. Coordinated IFC models were then generated using the COTS IFC export capability.

The models act as a test bed for the MVD and the COTS tools available at the time the work was performed. While the results will change with time, as software is updated to accommodate this and potentially other new MVDs, the procedures described here can be used by others to evaluate their software and design processes. 


\section{Experimental Building Models}

\subsection{Overview}

This chapter documents the procedure followed for the design-phase modeling and IFC export of water systems equipment found in three typical Army facilities: a Duplex Apartment, an Office Building and a Clinic. The goal of this process is to produce model files in the native COTS BIMauthoring software and IFC formats for the information exchange of water system design information. The three example models produced by this process can serve as reference data for future experimentation by ERDC and testing by software developers and end users.

The starting points for the modeling were previously developed architectural and mechanical/ electrical/plumbing (MEP) experimental models (East 2012a). These models were initially developed using Revit 2011, which was current at the time the models were created. In the present work, Revit 2013, Update Release 2, build 20121003_2115(x64), was used. As Revit versions are not backward compatible, the models produced for the current project cannot be used within Revit 2011.

It should be noted at the outset that this MVD is based on IFC4, which had not been officially approved at the time this work was undertaken. As stated in Chipman et al. 2013 ( $\mathrm{p}$ 49):

While most product geometry information was already welldefined within IFC version $2 \times 3$ and implemented by many vendors, there were many concepts that required some of the lesser-supported IFC data structures and some that required the expanded MEP scope in IFC version 4 to achieve adequate levels of detail... While realizing that many of these concepts were not supported by existing COTS software, the MVD has been defined to allow partial compliance for now, but with allowances to later relax or replace some requirements after testing models produced by existing software .

Therefore, one cannot fault Revit 2013 (the selected COTS software, which was designed to use IFC2x3) for not handling the IFC4 concepts. In fact, as 
noted below (section 2.6), the tests used to validate conformance to the MVD were relaxed to take account of the IFC4 concepts.

The Revit models were edited to remove non-water system interior elements so that the tests of the water system MVD would not contain extraneous information. Families and groups not related to testing the water system MVD were deleted from the model.

The following sections describe creating the Revit 2013 water system design models and exporting these models to IFC. Details on the modeling of each building type are given below in separate sections.

Table 1 provides statistical information for each of the three models.

Table 1. Model statistics.

\begin{tabular}{|l|l|l|l|}
\hline Model Statistics & Duplex & Office & Clinic \\
\hline Native file size & $23.8 \mathrm{MB}$ & $23.5 \mathrm{MB}$ & $34.9 \mathrm{MB}$ \\
\hline IFC file size & $31.3 \mathrm{MB}$ & $58 \mathrm{MB}$ & $92.4 \mathrm{MB}$ \\
\hline Building Area & $3,372 \mathrm{sq} \mathrm{ft}$ & $40,053 \mathrm{sq} \mathrm{ft}$ & $49,571 \mathrm{sq} \mathrm{ft}$ \\
\hline No. of Spaces & 21 & 99 & 266 \\
\hline No. of types & 24 & 24 & 36 \\
\hline No. of Components & 40 & 88 & 377 \\
\hline
\end{tabular}

\subsection{Determination of additional water system properties}

In order to meet the requirements of the Water System MVD, additional properties must be added to those already on the water system elements in the experimental Revit models. That additional properties are needed is not a fault in the software used or the original models - it simply underscores the notion that, as new information exchanges are defined, additional properties can be added to the models to accommodate the new uses that the new MVD provides.

The following procedure was used to determine the required properties:

1. Using the schedules in the Revit models, a spreadsheet listing the water system element types was created and the occurrence of each type in the three models was noted (Appendix A). 
2. A list of the property sets and properties for these element types was extracted from the MVD and added to the spreadsheet for each type.

3. The list of properties needed by the Water System MVD was compared to the list of properties already on the elements in the Revit models to create a list of the properties that needed to be added to the element types in the model. This list is shown in Appendix B.

The time needed to identify the needed properties will depend on the complexity of the model, but can take a day or two.

\subsection{Addition of water system properties to the Revit experimental building models}

The base architectural and MEP Revit models were downloaded from the Building Smart Alliance website, http:/ / buildingsmartalliance.org/ index.php/projects/commonbimfiles/.

The following steps were followed to update and expand these models:

1. Non-water system elements were removed from the models so that the IFC export would include only the target water system elements and the building architectural elements necessary to define the floors, rooms and spaces.

2. Additional water system elements were added as needed.

3. Additional properties were added to the water system elements as needed. This process can take a day or two, depending on the complexity of the model. It is recommended that a copy of the original model be retained, since if errors are made, it is often easier to revert to the original model than it is to remove the incorrect properties.

4. Default values were assigned to element parameters.

5. Project parameters were updated.

\subsubsection{Removing nonessential elements from the models}

Nonessential building interior features, such as furniture, casework, electrical elements and mechanical systems, were deleted from the model.

\subsubsection{Modeling additional water system elements}

Since the water systems in these buildings models are relatively completely developed, little additional modeling was needed. The main cause for 
additional modeling related to the fact that the original experimental BIMs used single Revit element types to represent objects that need to be treated as separate elements for water system analysis and validation of the MVD. As one example, the Revit M_Bath Tub: 1525 mmx760 mm - Private element includes hot and cold water connectors and a representation of a faucet. This faucet, however, does not have the properties and IFC categorization needed to properly export and validate. Therefore, separate faucet elements were used for the tubs, showers and sinks in the water system BIMs.

\subsubsection{Adding properties to elements in the models}

In order to include the water system properties that were identified in section 2.2 on the families in the building model, it was necessary to directly update the families in Revit with the new parameters.

The following procedure was used to add required parameters to the models:

1. The Revit MEP model for the Duplex Apartment-Duplex_MEP.rvt was opened in Revit 2013.

2. New Revit Shared Parameters for each of the new water system properties were added to the COBieSharedParameters.txt file using the Revit Manage tab > Shared Parameters command. The resulting shared parameter file was saved as COBieSharedParameters - Ontologies MVD-Water.txt.

3. The new Shared Parameters were added to selected element categories using a proprietary custom add-in program. (Steps 2 and 3 can also be done using the Revit Manage tab > Project Parameters $>$ Add command.)

4. A copy was made of the "COBie Types Schedule," a multi-category schedule in the Duplex Apartment Model file, re-named where necessary based on the corresponding Revit Category and updated to include the new parameters.

5. The new parameters were edited in the Schedule Properties dialog box to apply the parameters to the appropriate element categories in the model (Figure 1). 
Figure 1. Revit parameter properties dialog box showing the lamp efficiency property applied to lighting fixtures.

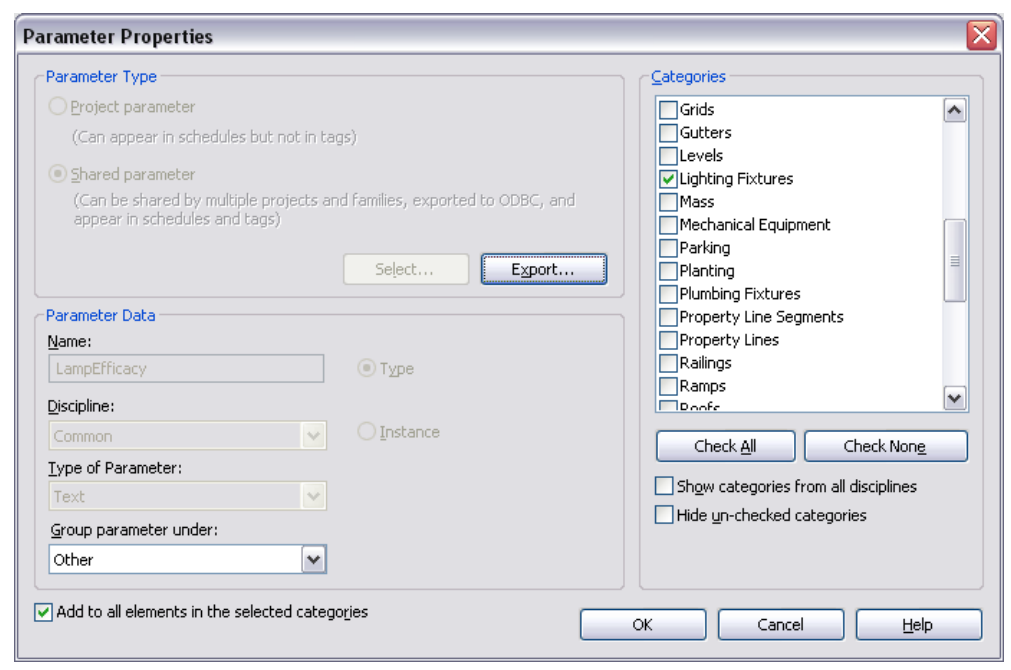

6. For the other models and the Revit Experimental BIM template (.RTE) files, each file was opened in Revit 2013 and the plumbing schedules created were copied into it from the first model. Copying the schedule into the model applies the shared parameters to the building elements in the model.

\subsubsection{Assigning default values}

Revit does not export to IFC custom parameters that do not have assigned values. Therefore, in order to be able to export all of the additional water system parameters to IFC, default values (such as 0 , UNSET, or N/A) were set using a proprietary custom add-in program. (Values could also have been set by editing the Revit schedules of the water system elements.)

\subsubsection{Updating project parameters}

The procedure for adding additional properties to the overall project were similar to the steps outlined in section 2.3.3, Adding Additional Properties to Elements in the Models. All shared parameters associated with the overall project were added as Project Parameters and associated with the Project Information category (see Figure 2). 
Figure 2. Revit Parameter Properties dialog box showing the building ID property applied to Project Information.

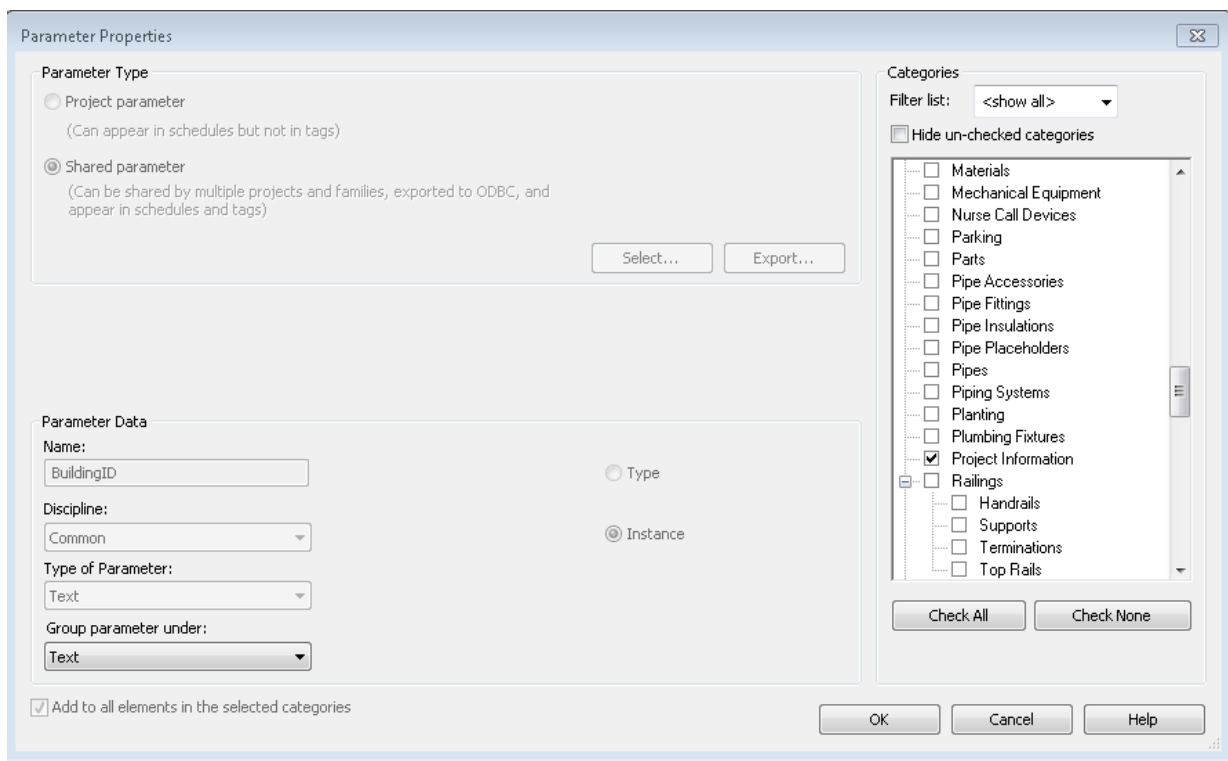

This category cannot be scheduled. These parameters were updated with the correct values by navigating to the Project Properties dialog box (Revit Manage tab > Project Information) and typing the values.

Figure 3. Revit Project Properties dialog box showing the building parameters.

\begin{tabular}{|c|c|c|c|c|}
\hline \multicolumn{4}{|c|}{ II. Project Properties } & $x$ \\
\hline Eamily: & \multicolumn{2}{|c|}{ System Family: Project Information } & \multicolumn{2}{|l|}{ Load... } \\
\hline Iype: & \multicolumn{2}{|c|}{ 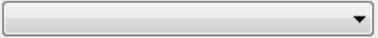 } & \multicolumn{2}{|l|}{ Edit Type... } \\
\hline \multicolumn{5}{|c|}{ Instance Parameters - Control selected or to-be-created instance } \\
\hline & Parameter & \multicolumn{2}{|c|}{ Value } & A \\
\hline \multicolumn{3}{|l|}{ Text } & $\hat{\imath}$ & \\
\hline \multicolumn{2}{|c|}{ BuildingID } & \multicolumn{2}{|l|}{ DuplexMEP } & \\
\hline \multicolumn{2}{|c|}{ ConstructionMethod } & \multicolumn{2}{|l|}{ New Construction } & \\
\hline \multicolumn{2}{|c|}{ FireProtectionClass } & \multicolumn{2}{|l|}{ Class 1} & \\
\hline \multicolumn{2}{|c|}{ GrossPlannedArea } & \multicolumn{2}{|l|}{312} & \\
\hline \multicolumn{2}{|c|}{ IsLandmarked } & \multicolumn{2}{|l|}{ FALSE } & $\equiv$ \\
\hline \multicolumn{2}{|c|}{ IsPermanentID } & \multicolumn{2}{|l|}{ FALSE } & \\
\hline \multicolumn{2}{|c|}{ OccupancyType } & \multicolumn{2}{|l|}{ Residential } & \\
\hline \multicolumn{2}{|c|}{ SprinklerProtection } & \multicolumn{2}{|l|}{ FALSE } & \\
\hline \multicolumn{2}{|c|}{ SprinklerProtectionAutomatic } & \multicolumn{2}{|l|}{ FALSE } & \\
\hline \multicolumn{2}{|c|}{ YearOfConstruction } & \multicolumn{2}{|l|}{$2011-2012$} & \\
\hline \multicolumn{2}{|c|}{ YearOfLastRefurbishment } & \multicolumn{2}{|l|}{0} & \\
\hline \multicolumn{2}{|c|}{ NetPlannedArea } & \multicolumn{2}{|l|}{500} & \\
\hline Electri & & 500 & & \\
\hline Fuel & & 200 & & \\
\hline Heat & & 250 & & \\
\hline Steam & & 100 & & \\
\hline Water & & 300 & & \\
\hline Numb & OfStoreys & 2 & & \\
\hline Refere & & Type $\times \sqrt{Z}$ & & \\
\hline Identi & Data & & $\hat{\imath}$ & $1-$ \\
\hline & & OK & Cancel & \\
\hline
\end{tabular}




\subsection{Export of the models to IFC}

Once the building models had been edited to expand the water system model and remove extraneous elements, new properties added to the models and applied to the building elements in the models, and default values assigned, the models were ready to export to IFC files.

\subsubsection{Revit IFC export}

The basic procedures for successfully exporting the Revit model to IFC are outlined in ERDC/ CERL CR-11-2 (J ohnson and Fallon 2011).

Because of changes in Revit's IFC export between the 2011 and 2013 releases, some of the values of the IfcExportAs and IfcExportType override parameters needed to be changed. (The Revit 2011 settings from J ohnson and Fallon [2011] for water system elements are reproduced in Table 2.)

Table 2. IFC export override parameter settings.

\begin{tabular}{|l|l|l|}
\hline Revit Family & IfcExportAs & IfcExportType \\
\hline M_Bath Tub.rfa & IfcSanitaryTerminalType & BATH \\
\hline $\begin{array}{l}\text { M_Drinking Fountain - Rectangular - } \\
\text { Wall Mounted.rfa }\end{array}$ & IfcSanitaryTerminalType & SANITARYFOUNTAIN \\
\hline M_Floor Drain - Round.rfa & IfcPipeFittingType & ENTRY \\
\hline M_Hot Water Boiler - 59-440 kW.rfa & IfcBoilerType & WATER \\
\hline M_Inline Pump - Circulator.rfa & IfcPumpType & CIRCULATOR \\
\hline M_Lavatory - Oval.rfa & IfcSanitaryTerminalType & SINK \\
\hline M_Roof Drain.rfa & IfcPipeFittingType & ENTRY \\
\hline M_Shower Stall - Rectangular.rfa & IfcSanitaryTerminalType & SHOWER \\
\hline M_Sink - Island - Single.rfa & IfcSanitaryTerminalType & SINK \\
\hline M_Sink - Kitchen - Double.rfa & IfcSanitaryTerminalType & SINK \\
\hline M_Sink - Work.rfa & IfcSanitaryTerminalType & SINK \\
\hline M_Urinal - Wall Hung.ffa & IfcSanitaryTerminalType & URINAL \\
\hline M_Water Closet - Flush Tank.rfa & IfcSanitaryTerminalType & WCSEAT \\
\hline $\begin{array}{l}\text { M_Water Closet - Flush Valve - Wall } \\
\text { Mounted.rfa }\end{array}$ & IfcSanitaryTerminalType & WCSEAT \\
\hline M_Water Heater.rfa & IfcTankType & PREFORMED \\
\hline
\end{tabular}

The Revit model was exported to IFC using the IFC-exportlayers.txt file created for the Experimental BIM project (East 2012a). The IFC- 
exportlayers.txt file provides additional Revit-to-IFC mappings to enable correct export of the Revit elements and properties to IFC (see J ohnson and Fallon 2011 for more details).

\subsubsection{Special considerations regarding Revit IFC export}

Several special considerations that apply to Revit's IFC export functionality affect the completeness of the IFC file and its validation against the MVD. It should be noted that since the version of Revit 2013 used in this work (20121003_2115 [x64]) was released, changes have been made to the open source Revit IFC export code. Users who wish to use the latest Revit IFC export code should get the latest version of the open source Revit exporter plug-in, either from SourceForge (http:// sourceforge.net/projects/ ifcexporter/ files/2013) or from the Autodesk Exchange App Store (http:/ / apps.exchange.autodesk.com/ RVT/ Home/ Index).

- Property values that should be numeric but that have a unit text, such as '208 V' for 208 volts, get incorrectly converted in the IFC export. For example, '208 V' exports as '2238.89'. This is a known issue in Revit. The Revit API GetParameter function returns values in a mixture of imperial units for length and metric units for everything else. So volts, which can be expressed as $\left(\mathrm{kg}^{*} \mathrm{~m}^{2}\right) /\left(\mathrm{A}^{*} \mathrm{~s}^{3}\right)$, get returned as $\left(\mathrm{kg} * \mathrm{ft}{ }^{2}\right)$ / $\left(\mathrm{A}^{*} \mathrm{~s}^{3}\right)$. Note that $2238.89 / 208$ is approximately 10.76 , or in other words $1 \mathrm{~m}^{2} / 1 \mathrm{ft}^{2}$ (Valdez 2012). This problem has reportedly been fixed in the Open Source code.

- At the time Revit 2013 was released, IFC4 had not been approved, so it is not supported. Because the water system MVD is written to take advantage of IFC4, adjustments were made to the validation tests to account for the fact that Revit exports to IFC 2x3.

\subsection{Validation of the Revit 2013 IFC export}

The exported IFC files for the three BIMs were checked against the Water System MVD using the ifcDoc tool (downloaded from http:// www.buildingsmart-tech.org/ specifications/specificationtools/ifodoc-tool). IfcDoc is a Microsoft Windows program that automates IFC documentation generation for both baseline IFC documentation as well as model view definitions. It supports generating boilerplate text from templates, hyperlinks, EXPRESS definitions, figures, tables, contents, and indices. It can also import MVD-XML and file and validate IFC files for conformance to that MVD. Additional information on out validation 
methodology is presented in Appendix C. Additional information on using the ifcDoc tool can be found at http:// www.buildingsmarttech.org/specifications/specification-tools/ifcdoc-tool/ifcdoc-help-page.

Because the water system MVD was based on IFC4, which was unofficial at the time this work was undertaken, Revit 2013 does not support IFC4 concepts. Adjustments to the requirements of the MVD were made in ifcDoc to make some IFC4 concepts optional. Other modifications were made to simplify the report format and combine similar error reports into single line items.

As model view definitions have historically been defined by their documentation only (no computer interpretable rules), validation has typically been a manual process requiring human interpretation of documentation and authoring of programming code to test IFC files.

BuildingSmart International established the mvdXML format as a way to automate the validation process such that future model view definitions may be validated without custom programming. The water system model view definition presented here was one of the first to leverage mvdXML. At the time this MVD was authored, mvdXML 1.0 had been finalized and approved, but validation tools were not readily available.

The Global Testing Documentation Server tool (gtds.buildingsmart.com; registration required) was scheduled to support mvdXML validation at the time of IFC4 final release (previously scheduled for J uly 2012), however the IFC release schedule was extended to accommodate ISO approval, with the result that the mvdXML validation tools were not yet integrated into GTDS as of late 2012, when the present work was completed.

The Open Source Building Information Modelserver (bimserver.org) also indicated intentions of supporting mvdXML validation, but that support was delayed due to resource availability. As of February 2013, a developer has actively been working on mvdXML integration. The timeline for this work required validation tools late in 2012. To accommodate this schedule, several alternatives were considered: (a) exclude validation efforts; (b) postpone validation until tools are available; or (c) invest resources out-ofpocket in creating such tools. The latter option was selected, and the researchers worked with buildingSmart International to expand the IfcDoc tool to support integrated validation of model view definitions. To help 
streamline other efforts, the utility was adapted to support integration with server tools such as those described above.

The report in Appendix C (Table C3) was generated by the IfcDoc validation tool, an open-source utility available at http:/ / www.buildingsmarttech.org/ mvd. The report is organized by IFC entity, with concepts underneath each entity. Columns are shown for on the right for each exchange, identified by number (see Table C1). The symbols in the column indicate the status of each test (see Table C2).

A small set of the concepts tested relied on IFC4, but are not found in IFC2x3. Ultimately, the goal of these model view definitions is to provide the necessary information to actually build a building. For example, port typing in particular is necessary to understand how equipment is connected, and to analyze systems for required capacity and conformance to building code; IFC4 supports identifying port types and system types unambiguously. Another such critical concept was classification. While that is also possible in IFC2x3, IFC4 provides an enforceable syntax to ensure that classifications are interoperable across files. Additionally, there were some specific IFC4 concepts leveraged for certain exchanges, such as occupancy schedules, projected energy usage, and resource allocations. To distinguish between IFC2x3 and IFC4 concepts, each IFC4-dependent concept is noted in Table $\mathrm{C} 3$ and overall statistics were calculated for both IFC2x3 and IFC4.

Ultimately, market-available software was found that could produce files $100 \%$ in conformance with some of the exchanges for IFC2x3. The more advanced concepts that failed the validation provide room to push the industry forward toward greater detail and better interoperability.

An overall summary of the MVD validation report is provided below in Table 3 and a detailed summary in Table C4. The percentages of noncompliant items are also provided and associated with the possible cause. Appendix C, Table C3, presents the validation results broken down by the specific exchange and Table $\mathrm{C} 4$ presents a summary of the numbers. The summary in Table 3 identifies the applicable concepts, number of passing concepts, and the percentage of concepts that passed the validation check, based on all of the object instances in the model. Table C3 shows the validation results based on each separate information exchange and presents 
percentages passing based on the number of rules checked, not the number of instances.

Table 3. IfcDoc MVD report summary.

\begin{tabular}{|l|l|l|l|}
\hline Water Systems MVD Validation & Duplex & Office & Clinic \\
\hline Tests Applicable & 408 & 408 & 408 \\
\hline Tests Passing All Exchanges & 368 & 368 & 373 \\
\hline Optional Tests Failing Any Exchange (number) & 22 & 22 & 18 \\
\hline Optional Tests Failing Any Exchange (percent) & $5.4 \%$ & $5.4 \%$ & $4.4 \%$ \\
\hline Mandatory Tests Failing Any Exchange (number) & 18 & 18 & 17 \\
\hline Mandatory Tests Failing Any Exchange (percent) & $4.4 \%$ & $4.4 \%$ & $4.2 \%$ \\
\hline Percent of Rules Passing (IFC4) & $95-99 \%$ & $95-99 \%$ & $96-99 \%$ \\
\hline Percent of Rules Passing (IFC2x3) & $97-100 \%$ & $97-100 \%$ & $97-100 \%$ \\
\hline Causes of Non-Compliance, as a percentage of total non-compliant concepts: & $61 \%$ & $61 \%$ \\
\hline IFC4 & $61 \%$ & $17 \%$ & $17 \%$ \\
\hline Revit IFC Export hard-coded to null & $17 \%$ & $22 \%$ & $22 \%$ \\
\hline Values not assigned in the code & $22 \%$ & $61 \%$ \\
\hline
\end{tabular}

As indicated in Table 3, about 5\% of the 408 tests failed for at least one optional exchange for the all three buildings; about 4\% failed at least one mandatory test for the Duplex Apartment and Office Building and about $10 \%$ for the Clinic. The overall failure rate for all tests, looked at for each exchange separately, ranged from $1 \%$ to $5 \%$ for IFC4 tests and from $0 \%$ to $3 \%$ for IFC2x3 tests. Not unexpectedly, none of the 19 exchanges passed all of the IFC4-related tests, but only 2 of the 19 exchanges passed all of the IFC2x3 tests.

Overall, the Entities (Item), Concepts (Sub-Items), and Problems related to the possible Causes of non-compliance are listed below in Table 4. Refer to Chapter 3 of Chipman et al. (2013) for discussion of Entities and Concepts as related to MVDs. 
Table 4. IfcDoc MVD non-compliance items.

\begin{tabular}{|c|c|c|c|}
\hline Item & Sub-ltem & Problem & Cause \\
\hline IfcSite & Site Location & $\begin{array}{l}\text { Physical address is needed for determining } \\
\text { applicable building codes }\end{array}$ & $\begin{array}{l}\text { Hard-coded to null in Revit IFC } \\
\text { export }\end{array}$ \\
\hline IfcBuilding & Classification & $\begin{array}{l}\text { OmniClass address needed for classifying building to } \\
\text { determine code requirements }\end{array}$ & IFC4 \\
\hline IfcBuilding & Product Assignment & $\begin{array}{l}\text { Tasks and resources are assigned to building } \\
\text { structures to indicate plumbing fixture requirements } \\
\text { in early design without specific product or placement } \\
\text { indicated. }\end{array}$ & $\begin{array}{l}\text { IFC4 } \\
\text { No support for tasks or resources } \\
\text { in Revit }\end{array}$ \\
\hline IfcBuilding & Building Location & Elevations needed to determine utility connectivity & $\begin{array}{l}\text { Hard-coded to null in Revit IFC } \\
\text { export }\end{array}$ \\
\hline IfcSpace & Classification & $\begin{array}{l}\text { OmniClass address needed for classifying space to } \\
\text { determine designed electrical usage per square foot }\end{array}$ & IFC4 \\
\hline IfcSanitaryTerminalType & Property Sets for Types & $\begin{array}{l}\text { Manufacturer type information necessary to identify, } \\
\text { order, and price products }\end{array}$ & $\begin{array}{l}\text { Variable created in Revit IFC export } \\
\text { code, but no values are assigned }\end{array}$ \\
\hline IfcSanitaryTerminalType & Type-Based Ports & Ports may be specified on types & $\begin{array}{l}\text { IFC4 } \\
\text { (Possibly in IFC2x3 but not } \\
\text { documented) }\end{array}$ \\
\hline IfcPipeFittingType & Property Sets for Types & $\begin{array}{l}\text { Manufacturer type information necessary to identify, } \\
\text { order, and price products }\end{array}$ & $\begin{array}{l}\text { Variable created in Revit IFC export } \\
\text { code, but no values are assigned }\end{array}$ \\
\hline IfcPipeSegmentType & Property Sets for Types & $\begin{array}{l}\text { Manufacturer type information necessary to identify, } \\
\text { order, and price products }\end{array}$ & $\begin{array}{l}\text { Variable created in Revit IFC export } \\
\text { code, but no values are assigned }\end{array}$ \\
\hline IfcPipeSegmentType & Material Profile Set & $\begin{array}{l}\text { Materials are placed in cross-sections of specified } \\
\text { dimensions following a path defined at occurrences } \\
\text { of the type }\end{array}$ & IFC4 \\
\hline IfcPumpType & Property Sets for Types & $\begin{array}{l}\text { Manufacturer type information necessary to identify, } \\
\text { order, and price products }\end{array}$ & $\begin{array}{l}\text { Variable created in Revit IFC export } \\
\text { code, but no values are assigned }\end{array}$ \\
\hline IfcPumpType & Type-Based Ports & Ports may be specified on types & $\begin{array}{l}\text { IFC4 } \\
\text { (Possibly in IFC2×3 but not } \\
\text { documented) }\end{array}$ \\
\hline IfcDistributionPort & Property Sets & $\begin{array}{l}\text { Port property sets indicate pipe connectivity, } \\
\text { including nominal diameter and connection types. }\end{array}$ & $\begin{array}{l}\text { IFC4 } \\
\text { (Possibly in IFC2×3 but not } \\
\text { documented) }\end{array}$ \\
\hline IfcDistributionPort & Material Profile Set Usage & $\begin{array}{l}\text { Material profiles indicate actual cross-sections and } \\
\text { the alignment axis for pipes and connections, } \\
\text { allowing for parametric layout of piping systems. }\end{array}$ & IFC4 \\
\hline IfcDistributionPort & Port Connectivity & $\begin{array}{l}\text { Port connectivity necessary to determine flow path } \\
\text { and calculate required design parameters (pressure, } \\
\text { temperature, volumetric flow rate). }\end{array}$ & $\begin{array}{l}\text { Object placement hard-coded to } \\
\text { null in Revit IFC export }\end{array}$ \\
\hline IfcValveType & Property Sets for Types & $\begin{array}{l}\text { Manufacturer type information necessary to identify, } \\
\text { order, and price products }\end{array}$ & $\begin{array}{l}\text { Variable created in Revit IFC export } \\
\text { code, but no values are assigned }\end{array}$ \\
\hline IfcValveType & Type-Based Ports & Ports may be specified on types & $\begin{array}{l}\text { IFC4 } \\
\text { (Possibly in IFC2x3 but not } \\
\text { documented) }\end{array}$ \\
\hline
\end{tabular}




\subsection{Issues}

Revit family categories are very broad currently. For example, only high level categories for plumbing fixtures are provided. The Plumbing Fixtures family category is intended to capture all plumbing fixture types i.e. toilets, sinks, drinking fountains, etc. The issue with this high level grouping arises when applying attributes from the shared parameters file to the fixtures. For example, attributes intended for a urinal were also applied to every other fixture (sinks for example) within the Plumbing Fixtures family category. This would require the user responsible for inputting values to either review the MVD documentation or have a thorough understanding of attributes associated with various plumbing equipment to understand which attributes require values. Although filters can be applied to schedules within Revit to hide non-applicable attributes for a particular fixture type, there is potential for non-applicable attributes to be overlooked and values inserted that would lead to incorrect data being exported out for a plumbing fixture type.

Being able to either create more family and/ or sub-categories and being allowed to apply shared parameter attributes at the sub-categories level would provide an efficient method for associating only the applicable attributes to various plumbing equipment objects. 


\section{Summary, Conclusions and Recommendations}

While the subject matter experts interviewed in the first stage of this project (Chipman et al. 2013) showed a diversity of project delivery methods and specific workflows, it was possible to define role-based information exchanges that were broadly applicable. The information exchanges identified in these workflows established the basis for developing the water systems MVD. Although IFC2x3 was the current standard at the time of this report, the water systems MVD was written for IFC4. IFC4 was used due to its expanded capabilities for describing interior plumbing schemas. The final version of IFC4 is scheduled to be released in 2013 and it is anticipated that the issues identified in this report related to IFC4 will be addressed once IFC4 is officially released and adopted by software vendors.

Using the water systems MVD, additional required properties were identified for the plumbing elements in the experimental BIMs and added to the models. Based on the results received after updating each of the BIMs with the additional MVD attributes, converting the BIMs to IFC, and analyzing the data utilizing the ifcDoc tool, it can be concluded that the current COTS version of Revit 2013, Update 2, is capable of capturing and managing the necessary information within the BIMs.

Although the water systems MVD is new and still preliminary, and thus not supported by COTS software, and Revit does not claim to have extensive IFC 2X3 export support for MEP systems, the validation reports generated by the ifcDoc tool indicated that almost two-thirds of the included concepts were successfully exported to IFC. The validation reports indicated that the three main causes for noncompliance were:

- concepts and properties introduced in IFC4

- property values that are hard-coded to null in Revit IFC export

- property values are not assigned in the Revit IFC export code.

During the process of adding MVD attributes to the models using the shared parameters method in Revit 2013, it was discovered that plumbing objects were too broadly categorized to effectively associate specific attributes with the appropriate components. This situation meant that attributes 
appropriate for a urinal, for example, would also be applied to other plumbing fixtures such as sinks or bathtubs.

In the process of conducting this research, it became clear that describing and maintaining the systems and connections between elements in the BIM is essential to extracting useful data for the information exchanges described by this MVD. This requirement has implications both for the way some plumbing elements are defined in the COTS software and for the way a typical BIM design project subdivides the model:

- Some BIM families (e.g., sinks) can belong to different systems and even different disciplines, and might be better modeled if broken into separate components. For sinks, this means that the body of the sink attaches to the waste water system, while one valve of the faucet attaches to the hot water system and the other to the cold water system.

- The common method of subdividing large projects by floors or even by parts of floors can sever the system connections between elements in different parts of the model. Without these system connections, some of the information needed for the information exchanges in the MVD is lost. New ways of subdividing large models are needed that will preserve both the system and the contextual information.

The modeling work described in this report demonstrates that it is possible, recognizing some limitations in the current software, to use the new MVD to add properties to the BIM and export that information to IFC, where it can be used for further analyses. If future software releases address the issues noted above, the BIMs will have the ability to achieve greater interoperability by providing the additional information that teams require during the design phase.

Looking to the future, although the IFC2x3 Coordination View has been sufficient for BIM use in the industry to date, it is time for vendors to support the entire IFC data model more fully. In that way, the ability of the software to handle new MVDs would be increased. Further, the software development tools and techniques accompanying IFC4, particularly mvdXML, make the incorporation of new MVDs much easier for software vendors.

In order to mitigate the problem of identifying additional properties required for product categories, methods are needed to align proprietary 
types, hierarchies, and inheritance with the IFC data model. These problems would also be reduced, and user experience and predictability of outcome would be enhanced, if the BIM content included in authoring software products and provided by manufacturers contained the relevant IFC property sets. 


\section{References}

Chipman, Tim, Kristine K. Fallon, Robert A. Feldman, Gregory Williams, and Omobolawa Fadojutimi. 2013. Ontology for Life-Cycle Modeling of Water Distribution Systems: Model View Definition. ERDC/ CERL CR-13-4. Champaign, IL: US Army Engineer Research and Development Center, Construction Engineering Research Laboratory (ERDC-CERL).

East, E.W. 2007. Construction Operations Building Information Exchange (COBIE), Requirements Definition and Pilot Implementation Standard, ERDC/ CERL TR07-30, US Army Corps of Engineers, http://www.wbdg.org/pdfs/erdc_cerl_tr0730.pdf. August 2007.

East, E. W. 2012a. "Common Building Information Model Files," buildingSMART alliance, National Institute of building Sciences, Washington, DC. http:// buildingsmartalliance.org/index.php/projects/commonbimfiles/, accessed 11 October 2012.

East, E.W. 2012b. Construction Operations Building Information Exchange (COBie), http:// www.wbdg.org/ resources/ cobie.php, accessed 6 August 2012.

East, E.W. 2012c. Equipment Layout information exchange (ELie). Available at: http:// www.buildingsmartalliance.org/index.php/ projects/activeprojects/ 114, accessed 17 August 2012.

East, E.W. 2012d. Water System information exchange (WSie). Available at http:// www.buildingsmartalliance.org/index.php/projects/activeprojects/ 179, accessed 17 August 2012.

East, E.W., Danielle Love and Nicholas Nisbet, 2010. A Life-Cycle Model for Contracted Information Exchange. Proceedings of the CIB W78 2010: 27th International Conference - Cairo, Egypt, 16-18 November 2010.

Hietanen, J . 2008. IFC Model View Definition Format. Available at: http://www.iaitech.org/downloads/ accompanyingdocuments/formats/MVD_Format_V2_Proposal_080128.pdf.

J ohnson, Mark, and Kristine K. Fallon. Experimental Building Information Models. ERDC/ CERL CR-11-2. Champaign, IL: US Army Engineer Research and Development Center, Construction Engineering Research Laboratory (ERDCCERL).

Nisbet, Nicholas, Dave McKay and Bill East, n.d. Water Usage System Information Exchange, Close-out Report. Unpublished draft manuscript, ERDC/ CERL TR08-DRAFT, US Army Corps of Engineers, Version 0.30, dated J une 2011.

Valdez, Angel, 2012. Discussion post in http:// sourceforge.net/ projects/ifcexporter/ discussion/general/thread/d8171b6 e, accessed 23 October 2012. 


\section{Appendix A: Water System Building Component Occurrences in Revit Models}

Table A1 Water system building component occurrences in the Revit models.

\begin{tabular}{|c|c|c|c|c|c|}
\hline \multicolumn{2}{|l|}{ - - - COMPONENT - - - } & \multirow{2}{*}{$\begin{array}{l}\text { TYPE } \\
\text { IFC }\end{array}$} & \multicolumn{3}{|c|}{ BUILDING OCCURRENCE } \\
\hline FAMILY:TYPE & IFC & & Duplex & Clinic & Office \\
\hline Bath Tub Faucet:PT & IfcValve & IfcValveType & $*$ & & \\
\hline $\begin{array}{l}\text { Fire Extinguisher Cabinet: } 305 \text { x } 215 \text { x } \\
813 \mathrm{~mm}: 305 \text { x } 215 \text { x } 813 \mathrm{~mm}\end{array}$ & IfcFlowTerminal & IfcSanitaryTerminalType & & $*$ & \\
\hline Kitchen Sink Faucet:Kitchen Sink Faucet & IfcValve & IfcValveType & $*$ & & \\
\hline Lavatory Faucet: Lavatory Faucet & IfcValve & IfcValveType & $*$ & * & \\
\hline M_Backflow Preventer - 15-50 mm:20 mm & IfcFlowController & IfcValveType & & $*$ & \\
\hline M_Backflow Preventer - $15-50$ mm:25 mm & IfcFlowController & IfcValveType & $*$ & & $*$ \\
\hline M_Backflow Preventer - 15-50 mm:50 mm & IfcFlowController & IfcValveType & & & $*$ \\
\hline M_Ball Valve - 50-150 mm:100 mm & IfcFlowController & IfcValveType & & * & \\
\hline M_Ball Valve - 50-150 mm:150 mm & IfcFlowController & IfcValveType & & $*$ & \\
\hline M_Ball Valve - $50-150 \mathrm{~mm}: 50 \mathrm{~mm}$ & IfcFlowController & IfcValveType & $*$ & $*$ & $*$ \\
\hline M_Ball Valve - 50-150 mm:65 mm & IfcFlowController & IfcValveType & & $*$ & \\
\hline M_Ball Valve - 50-150 mm:80 mm & IfcFlowController & IfcValveType & & $*$ & \\
\hline M_Bath Tub:1525 mmx760 mm - Private & IfcFlowTerminal & IfcSanitaryTerminalType & $*$ & & \\
\hline $\begin{array}{l}\text { M_Bend - PVC - Sch } 40 \text { - DWV:Bend - PVC - } \\
\text { Sch } 40 \text { - DWV }\end{array}$ & IfcPipeFitting & IfcPipeFittingType & $*$ & $*$ & * \\
\hline $\begin{array}{l}\text { M_Drinking Fountain - Rectangular - Wall } \\
\text { Mounted:Standard }\end{array}$ & IfcFlowTerminal & IfcSanitaryTerminalType & & $*$ & * \\
\hline M_Elbow - Generic: Elbow - Generic & IfcPipeFitting & IfcPipeFittingType & $*$ & * & $*$ \\
\hline $\begin{array}{l}\text { M_Floor Drain - Round: } 125 \text { mm Strainer - } \\
100 \mathrm{~mm} \text { Drain }\end{array}$ & IfcPipeFitting & IfcPipeFittingType & & $*$ & \\
\hline M_Hot Water Boiler - 59-440 kW:147 kW & IfcEnergyConversionDevice & IfcBoilerType & $*$ & & \\
\hline $\begin{array}{l}\text { M_Inline Pump - Circulator:3.9 LPS - } 0.8 \\
\text { Meter Head }\end{array}$ & IfcFlowMovingDevice & IfcPumpType & $*$ & $*$ & * \\
\hline $\begin{array}{l}\text { M_Lavatory - Oval: } 535 \text { mmx485 mm - } \\
\text { Private }\end{array}$ & IfcFlowTerminal & IfcSanitaryTerminalType & $*$ & & \\
\hline $\begin{array}{l}\text { M_Lavatory - Oval:535 mmx485 mm - } \\
\text { Public }\end{array}$ & IfcFlowTerminal & IfcSanitaryTerminalType & & $*$ & * \\
\hline $\begin{array}{l}\text { M_Lavatory - Oval: } 650 \mathrm{~mm} \times 485 \mathrm{~mm} \text { - } \\
\text { Private }\end{array}$ & IfcFlowTerminal & IfcSanitaryTerminalType & * & & \\
\hline $\begin{array}{l}\text { M_Roof Drain:380 mm Strainer - } 25 \mathrm{~mm} \\
\text { Drain }\end{array}$ & IfcPipeFitting & IfcPipeFittingType & $*$ & $*$ & * \\
\hline $\begin{array}{l}\text { M_Shower Stall - Rectangular:865 mmx815 } \\
\text { mm - Private }\end{array}$ & IfcFlowTerminal & IfcSanitaryTerminalType & $*$ & & \\
\hline $\begin{array}{l}\text { M_Shower Stall - Rectangular:915 mm×915 } \\
\text { mm - Private }\end{array}$ & IfcFlowTerminal & IfcSanitaryTerminalType & & & * \\
\hline $\begin{array}{l}\text { M_Shower Stall - Rectangular:915 mmx915 } \\
\text { mm - Public }\end{array}$ & IfcFlowTerminal & IfcSanitaryTerminalType & & $*$ & $*$ \\
\hline
\end{tabular}




\begin{tabular}{|c|c|c|c|c|c|}
\hline \multicolumn{2}{|l|}{ - - - COMPONENT - - - } & \multirow{2}{*}{$\begin{array}{l}\text { TYPE } \\
\text { IFC } \\
\end{array}$} & \multicolumn{3}{|c|}{ BUILDING OCCURRENCE } \\
\hline FAMILY:TYPE & IFC & & Duplex & Clinic & Office \\
\hline $\begin{array}{l}\text { M_Sink - Island - Single: } 455 \mathrm{mm \times 455} \mathrm{mm} \text { - } \\
\text { Private }\end{array}$ & IfcFlowTerminal & IfcSanitaryTerminalType & * & $*$ & \\
\hline $\begin{array}{l}\text { M_Sink - Kitchen - Double:1065 mmx535 } \\
\text { mm - Public }\end{array}$ & IfcFlowTerminal & IfcSanitaryTerminalType & & & * \\
\hline M_Sink - Mop:710 mmx710 mm & IfcFlowTerminal & IfcSanitaryTerminalType & & $*$ & \\
\hline M_Sink - Work:510 mmx455 mm & IfcFlowTerminal & IfcSanitaryTerminalType & & * & * \\
\hline $\begin{array}{l}\text { M_Sprinkler - Pendent - Hosted:15 mm } \\
\text { Pendent }\end{array}$ & IfcFlowTerminal & IfcFireSuppression TerminalType & & * & * \\
\hline M_Tee - Generic:Tee - Generic & IfcPipeFitting & IfcPipeFittingType & $*$ & $*$ & $*$ \\
\hline M_Transition - Generic:Transition - Generic & IfcPipeFitting & IfcPipeFittingType & & $*$ & $*$ \\
\hline M_Urinal - Wall Hung:25 mm Flush Valve & IfcFlowTerminal & IfcSanitaryTerminalType & & $*$ & $*$ \\
\hline $\begin{array}{l}\text { M_Water Closet - Flush Tank:Private - } 6.1 \\
\text { Lpf }\end{array}$ & IfcFlowTerminal & IfcSanitaryTerminalType & * & & \\
\hline $\begin{array}{l}\text { M_Water Closet - Flush Valve - Wall } \\
\text { Mounted:Public - } 6.1 \mathrm{Lpf}\end{array}$ & IfcFlowTerminal & IfcSanitaryTerminalType & & $*$ & * \\
\hline M_Water Heater:380 L & IfcFlowStorageDevice & IfcTankType & & $*$ & $*$ \\
\hline Pipe Types:Cold Water & IfcPipeSement & IfcPipeSementType & $*$ & $*$ & $*$ \\
\hline Pipe Types:Fire Protection & IfcPipeSement & IfcPipeSementType & & $*$ & \\
\hline Pipe Types:Hot Water & IfcPipeSement & IfcPipeSementType & $*$ & $*$ & $*$ \\
\hline Pipe Types:PVC & IfcPipeSement & IfcPipeSementType & $*$ & & \\
\hline Pipe Types:Storm & IfcPipeSement & IfcPipeSementType & & $*$ & $*$ \\
\hline Pipe Types:Waste & IfcPipeSement & IfcPipeSementType & $*$ & $*$ & $*$ \\
\hline $\begin{array}{l}\text { Shower-Faucet_Trim-DELTA-Linden- } \\
\text { T17294:Polished Chrome }\end{array}$ & IfcValve & IfcValveType & * & $*$ & \\
\hline
\end{tabular}




\section{Appendix B: Additional Water System Properties for Revit Families and Model}

Table B1. Additional water system properties for the Revit families.

\begin{tabular}{|c|c|c|c|}
\hline Family:Type & Revit Category & Ifc Category - MVD & Attributes \\
\hline M_Backflow Preventer - $15-50$ mm:20 mm & \multirow{3}{*}{ Pipe Accessories } & \multirow{3}{*}{ IfcValve } & Pset_ValveTypeCommon \\
\hline M_Backflow Preventer - $15-50$ mm:25 mm & & & Reference \\
\hline M_Backflow Preventer - 15-50 mm:50 mm & & & ValvePattern \\
\hline & & & ValveOperation \\
\hline & & & ValveMechanism \\
\hline & & & Size \\
\hline & & & BodyMaterial \\
\hline & & & TestPressure \\
\hline & & & WorkingPressure \\
\hline & & & FlowCoefficient \\
\hline & & & CloseRating \\
\hline & & & Pset_ValvePHistory \\
\hline & & & PercentageOpen \\
\hline & & & MeasuredFlowRate \\
\hline & & & MeasuredPressureDrop \\
\hline & & & Pset_ValveTypePressureReducing \\
\hline & & & UpstreamPressure (Instance) \\
\hline & & & DownstreamPressure (Instance) \\
\hline
\end{tabular}

\begin{tabular}{|c|c|c|c|}
\hline Family:Type & Revit Category & Ifc Category - MVD & Attributes \\
\hline M_Ball Valve - 50-150 mm:50 mm & \multirow{5}{*}{ Pipe Accessories } & \multirow{5}{*}{ IfcValve } & Pset_ValveTypeCommon \\
\hline M_Ball Valve - 50-150 mm:65 mm & & & Reference \\
\hline M_Ball Valve - 50-150 mm:80 mm & & & ValvePattern \\
\hline M_Ball Valve - 50-150 mm:100 mm & & & ValveOperation \\
\hline M_Ball Valve - 50-150 mm:150 mm & & & ValveMechanism \\
\hline & & & Size \\
\hline & & & BodyMaterial \\
\hline & & & TestPressure \\
\hline & & & WorkingPressure \\
\hline & & & FlowCoefficient \\
\hline & & & CloseRating \\
\hline & & & Pset_ValvePHistory \\
\hline & & & PercentageOpen \\
\hline & & & MeasuredFlowRate \\
\hline & & & MeasuredPressureDrop \\
\hline
\end{tabular}




\begin{tabular}{|c|c|c|c|}
\hline Family:Type & Revit Category & Ifc Category - MVD & Attributes \\
\hline Bath Tub Faucet:PT & \multirow{4}{*}{ Plumbing Fixtures } & \multirow{4}{*}{ IfcValve } & Pset_ValveTypeFaucet \\
\hline Lavatory Faucet: Lavatory Faucet & & & FaucetType \\
\hline Kitchen Sink Faucet:Kitchen Sink Faucet & & & FaucetOperation \\
\hline $\begin{array}{l}\text { Shower-Faucet_Trim-DELTA-Linden- } \\
\text { T17294:Polished Chrome }\end{array}$ & & & FaucetFunction \\
\hline & & & Finish \\
\hline & & & FaucetTopDescription \\
\hline & & & Pset_ValveTypeMixing \\
\hline & & & MixerControl \\
\hline & & & OutletConnectionSize \\
\hline & & & Pset_ValveTypeCommon \\
\hline & & & same as above \\
\hline
\end{tabular}

\begin{tabular}{|c|c|c|c|}
\hline Family:Type & Revit Category & Ifc Category - MVD & Attributes \\
\hline M_Sink - Island - Single:455 mmx455 mm & \multirow{4}{*}{ Plumbing Fixtures } & \multirow{4}{*}{ IfcSanitaryTerminal } & Pset_SanitaryTerminalTypeCommon \\
\hline $\begin{array}{l}\text { M_Sink - Kitchen - Double:1065 mm×535 } \\
\text { mm - Public }\end{array}$ & & & Reference \\
\hline M_Sink - Work:510 mmx455 mm & & & Pset_SanitaryTerminalTypeSink \\
\hline \multirow[t]{8}{*}{ M_Sink - Mop:710 mmx710 mm } & & & SinkType \\
\hline & & & Mounting \\
\hline & & & NominalLength \\
\hline & & & NominalWidth \\
\hline & & & NominalDepth \\
\hline & & & Color \\
\hline & & & DrainSize \\
\hline & & & MountingOffset \\
\hline
\end{tabular}

\begin{tabular}{|c|c|c|c|}
\hline Family:Type & Revit Category & $\begin{array}{l}\text { Ifc Category - } \\
\text { MVD }\end{array}$ & Attributes \\
\hline $\begin{array}{l}\text { M_Lavatory - Oval:535 mmx485 mm - } \\
\text { Private }\end{array}$ & \multirow{3}{*}{ Plumbing Fixtures } & \multirow{3}{*}{ IfcSanitaryTerminal } & Pset_SanitaryTerminalTypeCommon \\
\hline $\begin{array}{l}\text { M_Lavatory - Oval:650 mmx485 mm - } \\
\text { Private }\end{array}$ & & & Reference \\
\hline M_Lavatory - Oval:535 mmx485 mm - Public & & & Pset_SanitaryTerminalTypeWashHandBasin \\
\hline & & & WashHandBasinType \\
\hline & & & Mounting \\
\hline & & & NominalLength \\
\hline & & & NominalWidth \\
\hline & & & NominalDepth \\
\hline & & & Color \\
\hline & & & DrainSize \\
\hline & & & MountingOffset \\
\hline
\end{tabular}




\begin{tabular}{|c|c|c|c|}
\hline Family:Type & Revit Category & $\begin{array}{l}\text { Ifc Category - } \\
\text { MVD }\end{array}$ & Attributes \\
\hline $\begin{array}{l}\text { M_Drinking Fountain - Rectangular - Wall } \\
\text { Mounted:Standard }\end{array}$ & Plumbing Fixtures & IfcSanitaryTerminal & Pset_SanitaryTerminalTypeCommon \\
\hline & & & Reference \\
\hline & & & Pset_SanitaryTerminalTypeSanitaryFountain \\
\hline & & & FountainType \\
\hline & & & Mounting \\
\hline & & & NominalLength \\
\hline & & & NominalWidth \\
\hline & & & NominalDepth \\
\hline & & & Color \\
\hline & & & DrainSize \\
\hline
\end{tabular}

\begin{tabular}{|c|c|c|c|}
\hline Family:Type & Revit Category & Ifc Category - MVD & Attributes \\
\hline $\begin{array}{l}\text { M_Shower Stall - Rectangular:865 mmx815 } \\
\text { mm - Private }\end{array}$ & \multirow[t]{3}{*}{ Plumbing Fixtures } & \multirow[t]{3}{*}{ IfcSanitaryTerminal } & Pset_SanitaryTerminalTypeCommon \\
\hline $\begin{array}{l}\text { M_Shower Stall - Rectangular:915 mm×915 } \\
\text { mm - Private }\end{array}$ & & & Reference \\
\hline $\begin{array}{l}\text { M_Shower Stall - Rectangular:915 mmx915 } \\
\text { mm - Public }\end{array}$ & & & Pset_SanitaryTerminalTypeShower \\
\hline & & & ShowerType \\
\hline & & & HasTray \\
\hline & & & NominalLength \\
\hline & & & NominalWidth \\
\hline & & & NominalDepth \\
\hline & & & Color \\
\hline & & & ShowerHeadDescription \\
\hline & & & DrainSize \\
\hline
\end{tabular}

\begin{tabular}{|l|l|l|l|}
\hline Family:Type & Revit Category & Ifc Category - MVD & Attributes \\
\hline M_Bath Tub:1525 mmx760 mm - Private & Plumbing Fixtures & IfcSanitaryTerminal & Pset_SanitaryTerminalTypeCommon \\
\hline \multirow{3}{*}{} & & Reference \\
& & Pset_SanitaryTerminalTypeBath \\
& BathType \\
& & NominalLength \\
& NominalWidth \\
& & NominalDepth \\
& Color \\
& & DrainSize \\
& HasGrabHandles \\
\hline
\end{tabular}




\begin{tabular}{|c|c|c|c|}
\hline Family:Type & Revit Category & Ifc Category - MVD & Attributes \\
\hline $\begin{array}{l}\text { M_Water Closet - Flush Tank:Private - } 6.1 \\
\text { Lpf }\end{array}$ & \multirow[t]{2}{*}{ Plumbing Fixtures } & \multirow[t]{2}{*}{ IfcSanitaryTerminal } & Pset_SanitaryTerminalTypeCommon \\
\hline $\begin{array}{l}\text { M_Water Closet - Flush Valve - Wall } \\
\text { Mounted:Public - } 6.1 \text { Lpf }\end{array}$ & & & Reference \\
\hline & & & Pset_SanitaryTerminalTypeToiletPan \\
\hline & & & ToiletType \\
\hline & & & ToiletPanType \\
\hline & & & PanMounting \\
\hline & & & Color \\
\hline & & & SpilloverLevel \\
\hline & & & NominalLength \\
\hline & & & NominalWidth \\
\hline & & & NominalDepth \\
\hline
\end{tabular}

\begin{tabular}{|l|l|l|l|}
\hline Family:Type & Revit Category & Ifc Category - MVD & Attributes \\
\hline M_Urinal - Wall Hung:25 mm Flush Valve & Plumbing Fixtures & IfcSanitaryTerminal & Pset_SanitaryTerminalTypeCommon \\
\hline \multirow{2}{*}{} & & Reference \\
& Pset_SanitaryTerminalTypeUrinal \\
& UrinalType \\
& Mounting \\
& Color \\
& SpilloverLevel \\
& NominalLength \\
& NominalWidth \\
& & NominalDepth \\
\hline
\end{tabular}

\begin{tabular}{|c|c|c|c|}
\hline Family:Type & Revit Category & Ifc Category - MVD & Attributes \\
\hline $\begin{array}{l}\text { M_Inline Pump - Circulator:3.9 LPS - } 0.8 \\
\text { Meter Head }\end{array}$ & Mechanical Equipment & IfcPump & Pset_PumpOccurrence \\
\hline & & & ImpellerDiameter \\
\hline & & & BaseType \\
\hline & & & DriveConnectionType \\
\hline & & & Pset_PumpPHistory \\
\hline & & & MechanicalEfficiency \\
\hline & & & OverallEfficiency \\
\hline & & & PressureRise \\
\hline & & & RotationSpeed \\
\hline & & & Flowrate \\
\hline & & & Power \\
\hline & & & Pset_PumpTypeCommon \\
\hline & & & Reference \\
\hline & & & Status (Instance) \\
\hline & & & FlowRateRange \\
\hline & & & FlowResistanceRange \\
\hline
\end{tabular}




\begin{tabular}{|l|l|l|l|}
\hline Family:Type & Revit Category & Ifc Category - MVD & Attributes \\
\hline \multirow{3}{*}{$\begin{array}{l}\text { M_Inline Pump - Circulator:3.9 LPS - 0.8 } \\
\text { Meter Head (cont'd.) }\end{array}$} & $\begin{array}{l}\text { Mechanical Equipment } \\
\text { (cont'd.) }\end{array}$ & IfcPump (cont'd.) & TemperatureRange \\
\cline { 4 - 4 } & & & NetPositiveSuctionHead \\
\cline { 3 - 4 } & & NominalRotationSpeed \\
\hline
\end{tabular}

\begin{tabular}{|c|c|c|c|}
\hline Family:Type & Revit Category & Ifc Category - MVD & Attributes \\
\hline M_Hot Water Boiler - 59-440 kW:147 kW & \multirow[t]{2}{*}{ Mechanical Equipment } & \multirow[t]{2}{*}{ IfcBoiler } & Pset_BoilerPHistory \\
\hline M_Water Heater:380 L & & & EnergySourceConsumption \\
\hline & & & OperationalEfficiency \\
\hline & & & CombustionEfficiency \\
\hline & & & WorkingPressure \\
\hline & & & CombustionTemperature \\
\hline & & & PartLoadRatio \\
\hline & & & Load \\
\hline & & & PrimaryEnergyConsumption \\
\hline & & & AuxiliaryEnergyConsumption \\
\hline & & & Pset_BoilerTypeCommon \\
\hline & & & Reference \\
\hline & & & Status (Instance) \\
\hline & & & PressureRating \\
\hline & & & OperatingMode \\
\hline & & & HeatTransferSurfaceArea \\
\hline & & & NominalPartLoadRatio \\
\hline & & & WaterInletTemperatureRange \\
\hline & & & WaterStorageCapacity \\
\hline & & & IsWaterStorageHeater \\
\hline & & & PartialLoadEfficiencyCurves \\
\hline & & & OutletTemperatureRange \\
\hline & & & NominalEnergyConsumption \\
\hline & & & EnergySource \\
\hline & & & Pset_BoilerTypeWater \\
\hline & & & NominalEfficiency \\
\hline & & & HeatOutput \\
\hline
\end{tabular}




\begin{tabular}{|c|c|c|c|}
\hline Family:Type & Revit Category & Ifc Category - MVD & Attributes \\
\hline $\begin{array}{l}\text { M_Roof Drain:380 mm Strainer - } 25 \text { mm } \\
\text { Drain }\end{array}$ & \multirow[t]{2}{*}{ Mechanical Equipment } & \multirow[t]{2}{*}{ IfcWasteTerminalType } & Pset_WasteTerminalTypeCommon \\
\hline $\begin{array}{l}\text { M_Floor Drain - Round:125 mm Strainer - } \\
100 \mathrm{~mm} \text { Drain }\end{array}$ & & & Reference \\
\hline & & & Status (Instance) \\
\hline & & & Pset_WasteTerminalTypeFloorWaste \\
\hline & & & NominalLength \\
\hline & & & NominalWidth \\
\hline & & & NominalDepth \\
\hline & & & OutletConnectionSize \\
\hline & & & CoverLength \\
\hline & & & CoverWidth \\
\hline & & & Pset_WasteTerminalTypeFloorWaste \\
\hline & & & NominalLength \\
\hline & & & NominalWidth \\
\hline & & & NominalDepth \\
\hline & & & OutletConnectionSize \\
\hline & & & CoverLength \\
\hline & & & CoverWidth \\
\hline
\end{tabular}

\begin{tabular}{|c|c|c|c|}
\hline Family:Type & Revit Category & Ifc Category - MVD & Attributes \\
\hline M_Elbow - Generic: Elbow - Generic & \multirow[b]{2}{*}{ Pipe Fittings } & \multirow[b]{2}{*}{ IfcPipeFitting } & Pset_PipeFittingTypeCommon \\
\hline M_Transition - Generic:Transition - Generic & & & Reference \\
\hline & & & Status (Instance) \\
\hline & & & PressureClass \\
\hline & & & PressureRange \\
\hline & & & TemperatureRange \\
\hline & & & FittingLossFactor \\
\hline & & & Pset_PipeFittingOccurrence \\
\hline & & & InteriorRoughnessCoefficient \\
\hline & & & Color \\
\hline & & & Pset_PipeFittingPHistory \\
\hline & & & LossCoefficient \\
\hline & & & FlowrateLeakage \\
\hline
\end{tabular}

\begin{tabular}{|l|l|l|l|}
\hline Family:Type & Revit Category & Ifc Category - MVD & Attributes \\
\hline $\begin{array}{l}\text { M_Bend - PVC - Sch 40 - DWV:Bend - PVC - } \\
\text { Sch 40 - DWV }\end{array}$ & Pipe Fittings & IfcPipeFitting & \multirow{2}{*}{ Pset_PipeFittingTypeCommon } \\
\hline \multirow{2}{*}{} & & Same as Above \\
& & Pset_PipeFittingTypeBend \\
& & BendAngle \\
& & BendRadius \\
\hline
\end{tabular}




\begin{tabular}{|c|c|c|c|}
\hline Family:Type & Revit Category & Ifc Category - MVD & Attributes \\
\hline M_Tee - Generic:Tee - Generic & Pipe Fittings & IfcPipeFitting & Pset_PipeFittingTypeCommon \\
\hline & & & Same as Above \\
\hline & & & Pset_PipeFittingTypeJunction \\
\hline & & & JunctionType \\
\hline & & & JunctionLeftAngle \\
\hline & & & JunctionLeftRadius \\
\hline & & & JunctionRightAngle \\
\hline & & & JunctionRightRadius \\
\hline
\end{tabular}

\begin{tabular}{|c|c|c|c|}
\hline Family:Type & Revit Category & Ifc Category - MVD & Attributes \\
\hline Pipe Types: PVC & \multirow{6}{*}{ Pipes } & \multirow{6}{*}{ IfcPipeSegment } & Pset_PipeSegmentTypeCommon \\
\hline Pipe Types:Waste & & & Reference \\
\hline Pipe Types:Cold Water & & & Status (Instance) \\
\hline Pipe Types:Hot Water & & & WorkingPressure \\
\hline Pipe Types:Fire Protection & & & PressureRange \\
\hline \multirow[t]{12}{*}{ Pipe Types:Storm } & & & TemperatureRange \\
\hline & & & NominalDiameter \\
\hline & & & InnerDiameter \\
\hline & & & OuterDiameter \\
\hline & & & Pset_PipeSegmentOccurrence \\
\hline & & & InteriorRoughnessCoefficient \\
\hline & & & Color \\
\hline & & & Gradient \\
\hline & & & InvertElevation \\
\hline & & & Pset_PipeSegmentPHistory \\
\hline & & & LeakageCurve \\
\hline & & & FluidFlowLeakage \\
\hline
\end{tabular}


Table B2. Additional project properties for to the file not associated with individual elements

\begin{tabular}{|c|c|c|}
\hline Revit Category & Ifc Category - MVD & $\begin{array}{l}\text { ATTRIBUTES } \\
\text { Pset_BuildingCommon }\end{array}$ \\
\hline \multirow[t]{14}{*}{ N/A } & \multirow[t]{14}{*}{ IfcBuiliding } & Reference \\
\hline & & BuildingID \\
\hline & & IsPermanentID \\
\hline & & ConstructionMethod \\
\hline & & FireProtectionClass \\
\hline & & SprinklerProtection \\
\hline & & SprinklerProtectionAutomatic \\
\hline & & OccupancyType \\
\hline & & GrossPlannedArea \\
\hline & & NetPlannedArea \\
\hline & & NumberOfStoreys \\
\hline & & YearOfConstruction \\
\hline & & YearOfLastRefurbishment \\
\hline & & IsLandmarked \\
\hline
\end{tabular}




\section{Appendix C: Sample Validation Report}

Table C1. Water system exchanges.

\begin{tabular}{|l|l|}
\hline Number & Exchange Name \\
\hline 1 & Facility Occupancy Model \\
\hline 2 & Compare System Options \\
\hline 3 & Locate Plumbing Fixtures \\
\hline 4 & Plumbing Equipment Requirements \\
\hline 5 & Plumbing Spatial Requirements \\
\hline 6 & Locate and Size Plumbing Equipment Rooms \\
\hline 7 & Specify Plumbing System Performance \\
\hline 8 & Size Plumbing System \\
\hline 9 & Plumbing Basis of Design \\
\hline 10 & Document Plumbing Design Schematic \\
\hline 11 & Coordinate With Other Building Systems \\
\hline 12 & Facility Spatial Configuration \\
\hline 13 & Water Supply Requirements \\
\hline 14 & Calculate Water Balance \\
\hline 15 & Piping Schematic \\
\hline 16 & Layout Plumbing System \\
\hline 17 & Pocument Coordinated Design and Equipment Sizes \\
\hline 19 & \\
\hline
\end{tabular}


Table C2 Validation test results symbols used in Table C3.

\begin{tabular}{|c|c|c|}
\hline Symbol & Meaning & Flag Column \\
\hline- & $\begin{array}{l}\text { The test was inapplicable for the particular } \\
\text { exchange }\end{array}$ & $\begin{array}{l}\text { A “-" indicates that the test does not apply to any of the } \\
\text { exchanges }\end{array}$ \\
\hline+ & $\begin{array}{l}\text { The entity or concept passed the test for } \\
\text { the given exchange }\end{array}$ & $\begin{array}{l}\text { A “+ indicates that the test passes for all applicable } \\
\text { exchanges }\end{array}$ \\
\hline * & $\begin{array}{l}\text { An optional rule for the particular } \\
\text { exchange failed the test }\end{array}$ & $\begin{array}{l}\text { An “*” indicates that the optional test failed at least one } \\
\text { of the applicable exchanges }\end{array}$ \\
\hline $\mathrm{F}$ & $\begin{array}{l}\text { A mandatory rule for the particular } \\
\text { exchange failed the test }\end{array}$ & $\begin{array}{l}\text { An "F" indicates that the mandatory test failed at least } \\
\text { one of the applicable exchanges }\end{array}$ \\
\hline $\mathrm{R}$ & Required concept (Req column) & \\
\hline 0 & Optional concept (Req column) & \\
\hline$\#<$ number $>$ & $\begin{array}{l}\text { The number identifies the first object } \\
\text { instance that violates the rule - the } \\
\text { numbers correspond to those in the IFC } \\
\text { file. (Errors column) }\end{array}$ & \\
\hline <number> & $\begin{array}{l}\text { Number of instances subject to the rule } \\
\text { (Count column) }\end{array}$ & \\
\hline
\end{tabular}

\section{Notes:}

1. The "Count" column in Table C3 indicates the number of instances subject to the rule.

2. The "Flag" column in Table C3 indicates if any of the exchanges have failures. 
Table C3. Clinic Water System MVD IFC validation.

\begin{tabular}{|c|c|c|c|c|c|c|c|c|c|c|c|c|c|c|c|c|c|c|c|c|c|c|c|}
\hline Entity/Concept & Req & Errors & Count & Flag & 1 & 2 & 3 & 4 & 5 & 6 & 7 & 8 & $\mathbf{9}$ & $\begin{array}{l}1 \\
0\end{array}$ & $\begin{array}{l}1 \\
1\end{array}$ & $\begin{array}{l}1 \\
2\end{array}$ & $\begin{array}{l}1 \\
3\end{array}$ & $\begin{array}{l}1 \\
4\end{array}$ & $\begin{array}{l}1 \\
5\end{array}$ & $\begin{array}{l}1 \\
6\end{array}$ & & $\begin{array}{l}1 \\
8\end{array}$ & $\begin{array}{l}1 \\
9\end{array}$ \\
\hline IfcProject & & & 1 & & & & & & & & & & & & & & & & & & & & \\
\hline Identity & $\mathrm{R}$ & & 1 & + & + & + & + & + & + & + & + & + & + & + & + & + & + & + & + & + & & + & + \\
\hline Spatial Decomposition & $\mathrm{R}$ & & & & & & & & & & & & & & & & & & & & & & \\
\hline Spatial Parts=IfcSite; & & & 1 & + & + & + & + & + & + & + & + & + & + & + & + & + & + & + & + & + & & + & + \\
\hline Project Units & $\mathrm{O}$ & & & & & & & & & & & & & & & & & & & & & & \\
\hline NamedUnitType=LENGTHUNIT; & & $\# 53$ & 0 & * & * & $*$ & * & * & * & * & * & * & * & * & * & * & * & * & * & * & 7 & * & * \\
\hline NamedUnitType=TIMEUNIT; & & \#53 & 0 & * & * & * & * & * & * & * & * & * & * & * & * & * & * & * & * & * & & * & * \\
\hline NamedUnitType=FREQUENCYUNIT; & & \#53 & 0 & * & * & * & * & * & * & * & * & * & * & * & * & * & * & * & * & * & s & * & * \\
\hline NamedUnitType=AREAUNIT; & & $\# 53$ & 0 & * & * & * & * & * & * & * & * & * & * & * & * & * & * & * & * & * & & * & * \\
\hline $\begin{array}{l}\text { NamedUnitType=THERMODYNAMICTE } \\
\text { MPERATUREUNIT; }\end{array}$ & & \#53 & 0 & * & * & * & * & * & * & * & * & * & * & * & * & * & * & * & * & * & & * & * \\
\hline NamedUnitType=PRESSUREUNIT; & & $\# 53$ & 0 & * & * & * & * & * & * & * & * & * & * & * & * & * & * & * & * & * & & * & * \\
\hline NamedUnitType=VOLUMEUNIT; & & $\# 53$ & 0 & * & * & * & * & * & * & * & * & * & * & * & * & * & * & * & * & * & & * & * \\
\hline Project Context & $\mathrm{O}$ & & & & & & & & & & & & & & & & & & & & & & \\
\hline $\begin{array}{l}\text { Contextldentifier=Plan; } \\
\text { ContextType=2D; }\end{array}$ & & & 1 & + & + & + & + & + & + & + & + & + & + & + & + & + & + & + & + & + & & + & + \\
\hline $\begin{array}{l}\text { ContextIdentifier=Model; } \\
\text { ContextType=3D; }\end{array}$ & & & 1 & + & + & + & + & + & + & + & + & + & + & + & + & + & + & + & + & + & & + & + \\
\hline Project Declaration [IFC4] & $\mathrm{O}$ & & & & & & & & & & & & & & & & & & & & & & \\
\hline Type=IfcSpaceType; & & $\# 53$ & 0 & * & * & $*$ & * & * & * & * & * & * & * & * & * & * & * & * & * & * & & * & * \\
\hline Type=IfcOccupant; & & $\# 53$ & 0 & * & * & $*$ & * & * & * & * & * & * & * & * & * & * & * & * & * & * & & * & * \\
\hline Type=IfcActor; & & $\# 53$ & 0 & * & * & * & * & * & * & * & * & * & * & * & * & * & * & * & * & * & & * & * \\
\hline Type=IfcSpace; & & \#53 & 0 & * & * & $*$ & * & * & * & * & * & * & * & * & * & * & * & * & * & * & & * & * \\
\hline Type=IfcWorkPlan; & & $\# 53$ & 0 & * & * & $*$ & * & * & * & * & * & * & * & * & * & * & * & * & * & * & & * & $*$ \\
\hline Type=IfcDistributionElementType; & & $\# 53$ & 0 & * & * & * & * & * & * & * & * & * & * & * & * & * & * & * & * & * & & * & * \\
\hline Type=IfcDistributionSystem; & & $\# 53$ & 0 & * & * & * & * & * & * & * & * & * & * & * & * & * & * & * & * & * & & * & * \\
\hline IfcSite & & & 1 & & & & & & & & & & & & & & & & & & & & \\
\hline Identity & $\mathrm{R}$ & & 1 & + & + & + & + & + & + & + & + & + & + & + & + & + & + & + & + & + & & + & + \\
\hline Site Location & $\mathrm{R}$ & $\begin{array}{l}\# 2448 \\
304\end{array}$ & 0 & $\mathbf{F}$ & * & * & * & * & * & * & * & * & * & * & * & $*$ & $F$ & * & * & * & & * & $F$ \\
\hline
\end{tabular}




\begin{tabular}{|c|c|c|c|c|c|c|c|c|c|c|c|c|c|c|c|c|c|c|c|c|c|c|c|}
\hline Entity/Concept & Req & Errors & Count & Flag & 1 & 2 & 3 & 4 & 5 & 6 & 7 & 8 & 9 & $\begin{array}{l}1 \\
0\end{array}$ & $\begin{array}{l}1 \\
1\end{array}$ & $\begin{array}{l}1 \\
2\end{array}$ & $\begin{array}{l}1 \\
3\end{array}$ & $\begin{array}{l}1 \\
4\end{array}$ & $\begin{array}{l}1 \\
5\end{array}$ & $\begin{array}{l}1 \\
6\end{array}$ & $\begin{array}{l}1 \\
7\end{array}$ & $\begin{array}{l}1 \\
8\end{array}$ & $\begin{array}{l}1 \\
9\end{array}$ \\
\hline Spatial Decomposition & $\mathrm{R}$ & & & & & & & & & & & & & & & & & & & & & & \\
\hline Spatial Parts=IfcBuilding; & & & 1 & + & + & + & + & + & + & + & + & + & + & + & + & + & + & + & + & + & + & + & + \\
\hline Footprint Geometry & $\mathrm{O}$ & & & & & & & & & & & & & & & & & & & & & & \\
\hline $\begin{array}{l}\text { RepresentationType=GeometricCurveSet } \\
\text {; Geometry=IfcGeometricCurveSet; }\end{array}$ & & $\begin{array}{l}2448 \\
304\end{array}$ & 0 & * & * & * & * & * & * & * & * & * & * & * & * & * & * & * & * & * & * & * & * \\
\hline $\begin{array}{l}\text { RepresentationType=Annotation2D; } \\
\text { Geometry=IfcAnnotationFillArea; }\end{array}$ & & $\begin{array}{l}\# 2448 \\
304\end{array}$ & 0 & * & * & * & * & * & * & * & * & * & * & * & * & * & * & * & * & * & * & * & * \\
\hline IfcBuilding & & & 1 & & & & & & & & & & & & & & & & & & & & \\
\hline Identity & $\mathrm{R}$ & & 1 & + & + & + & + & + & + & + & + & + & + & + & + & + & + & + & + & + & + & + & + \\
\hline Classification [IFC4] & $\mathrm{R}$ & & & & & & & & & & & & & & & & & & & & & & \\
\hline $\begin{array}{l}\text { Source=CSI; Name=OmniClass; To- } \\
\text { kens=11-00 } 0000 \text {; }\end{array}$ & & $\# 57$ & 0 & $\mathbf{F}$ & $\mathbf{F}$ & $\mathbf{F}$ & $\mathbf{F}$ & $F$ & $\mathbf{F}$ & $F$ & $\mathbf{F}$ & $\mathbf{F}$ & $F$ & $\mathbf{F}$ & $\mathbf{F}$ & $\mathbf{F}$ & $F$ & $\mathbf{F}$ & $\mathbf{F}$ & $\mathbf{F}$ & $\mathbf{F}$ & $\mathbf{F}$ & $\mathbf{F}$ \\
\hline Property Sets & $\mathrm{O}$ & & & & & & & & & & & & & & & & & & & & & & \\
\hline Name=Pset_BuildingCommon; & & $\# 57$ & 0 & * & * & * & * & * & * & * & * & * & * & * & * & * & * & * & * & * & * & * & * \\
\hline Product Assignment & $\mathrm{R}$ & & & & & & & & & & & & & & & & & & & & & & \\
\hline Type=IfcTask; & & \#57 & 0 & $F$ & $\mathbf{F}$ & - & - & - & - & - & - & - & - & - & - & - & - & - & - & - & - & - & - \\
\hline Spatial Decomposition & $\mathrm{R}$ & & & & & & & & & & & & & & & & & & & & & & \\
\hline Spatial Parts=IfcBuildingStorey; & & & 1 & + & + & + & + & + & + & + & + & + & + & + & + & + & + & + & + & + & + & + & + \\
\hline Building Location & $\mathrm{R}$ & \#57 & 0 & $F$ & * & $\mathbf{F}$ & * & $F$ & * & * & * & * & * & $*$ & * & * & $F$ & * & * & * & * & * & $F$ \\
\hline IfcTask & & & 0 & & & & & & & & & & & & & & & & & & & & \\
\hline Identity & $\mathrm{R}$ & & 0 & + & + & - & - & - & - & - & - & - & - & - & - & - & - & - & - & - & - & - & - \\
\hline Process Assignment & $\mathrm{R}$ & & & & & & & & & & & & & & & & & & & & & & \\
\hline Type=IfcSubContractResource; & & & 0 & + & + & - & - & - & - & - & - & - & - & - & - & - & - & - & - & - & - & - & - \\
\hline Type= IfcConstructionProductResource; & & & 0 & + & + & - & - & - & - & - & - & - & - & - & - & - & - & - & - & - & - & - & - \\
\hline IfcConstructionProductResource & & & 0 & & & & & & & & & & & & & & & & & & & & \\
\hline Identity & $\mathrm{R}$ & & 0 & + & + & - & - & - & - & - & - & - & - & - & - & - & - & - & - & - & - & - & - \\
\hline Resource Cost & $\mathrm{O}$ & & & & & & & & & & & & & & & & & & & & & & \\
\hline $\begin{array}{l}\text { CostType=IfcCostValue; } \\
\text { CostName=Product; } \\
\text { ValueType=IfcMonetaryMeasure; }\end{array}$ & & & 0 & + & + & - & - & - & - & - & - & - & - & - & - & - & - & - & - & - & - & - & - \\
\hline
\end{tabular}




\begin{tabular}{|c|c|c|c|c|c|c|c|c|c|c|c|c|c|c|c|c|c|c|c|c|c|c|c|}
\hline Entity/Concept & Req & Errors & Count & Flag & 1 & 2 & 3 & 4 & 5 & 6 & 7 & 8 & 9 & $\begin{array}{l}1 \\
0\end{array}$ & $\begin{array}{l}1 \\
1\end{array}$ & $\begin{array}{l}1 \\
2\end{array}$ & $\begin{array}{l}1 \\
3\end{array}$ & $\begin{array}{l}1 \\
4\end{array}$ & $\begin{array}{l}1 \\
5\end{array}$ & $\begin{array}{l}1 \\
6\end{array}$ & $\begin{array}{l}1 \\
7\end{array}$ & $\begin{array}{l}1 \\
8\end{array}$ & $\begin{array}{l}1 \\
9\end{array}$ \\
\hline $\begin{array}{l}\text { CostType=IfcCostValue; } \\
\text { CostName=Shipping; } \\
\text { ValueType=IfcMonetaryMeasure; }\end{array}$ & & & 0 & + & + & - & - & - & - & - & - & - & - & - & - & - & - & - & - & - & - & - & - \\
\hline Resource Quantity & $\mathrm{R}$ & & & & & & & & & & & & & & & & & & & & & & \\
\hline $\begin{array}{l}\text { QuantityType=IfcQuantityCount; } \\
\text { QuantityName=Count; }\end{array}$ & & & 0 & + & + & - & - & - & - & - & - & - & - & - & - & - & - & - & - & - & - & - & - \\
\hline Resource Assignment & $\mathrm{R}$ & & & & & & & & & & & & & & & & & & & & & & \\
\hline Type=IfcSanitaryTerminal; & & & 0 & + & + & - & - & - & - & - & - & - & - & - & - & - & - & - & - & - & - & - & - \\
\hline IfcOccupant & & & 0 & & & & & & & & & & & & & & & & & & & & \\
\hline Identity & $\mathrm{R}$ & & 0 & + & + & - & - & - & - & - & - & - & - & - & - & - & - & - & - & - & - & - & - \\
\hline Actor Assignment & $\mathrm{O}$ & & & & & & & & & & & & & & & & & & & & & & \\
\hline Type=IfcSpatialStructureElement; & & & 0 & + & + & - & - & - & - & - & - & - & - & - & - & - & - & - & - & - & - & - & - \\
\hline Type=IfcWorkCalendar; & & & 0 & + & + & - & - & - & - & - & - & - & - & - & - & - & - & - & - & - & - & - & - \\
\hline Property Sets & - & & 0 & - & - & - & - & - & - & - & - & - & - & - & - & - & - & - & - & - & - & - & - \\
\hline Contact & - & & 0 & - & - & - & - & - & - & - & - & - & - & - & - & - & - & - & - & - & - & - & - \\
\hline IfcActor & & & 0 & & & & & & & & & & & & & & & & & & & & \\
\hline Identity & $\mathrm{R}$ & & 0 & + & - & + & - & - & - & - & - & - & - & - & - & - & - & - & - & - & - & - & - \\
\hline Property Sets & $\mathrm{O}$ & & & & & & & & & & & & & & & & & & & & & & \\
\hline $\begin{array}{l}\text { Name=Pset_ActorCommon; } \\
\text { TemplateType= } \\
\text { PSET_OCCURRENCEDRIVEN; }\end{array}$ & & & 0 & + & - & + & - & - & - & - & - & - & - & - & - & - & - & - & - & - & - & - & - \\
\hline Contact & $\mathrm{R}$ & & & & & & & & & & & & & & & & & & & & & & \\
\hline $\begin{array}{l}\text { Role=USERDEFINED; } \\
\text { UserDefinedRole=ElectricalUtility; }\end{array}$ & & & 0 & + & - & + & - & - & - & - & - & - & - & - & - & - & - & - & - & - & - & - & - \\
\hline Actor Assignment & $\mathrm{R}$ & & & & & & & & & & & & & & & & & & & & & & \\
\hline Type=IfcWorkPlan; & & & 0 & + & - & + & - & - & - & - & - & - & - & - & - & - & - & - & - & - & - & - & - \\
\hline IfcSanitaryTerminal & & & 0 & & & & & & & & & & & & & & & & & & & & \\
\hline Identity & $\mathrm{R}$ & & 0 & + & - & + & + & + & + & + & + & + & + & + & + & + & + & + & + & + & + & + & + \\
\hline Object Typing & $\mathrm{R}$ & & 0 & + & - & - & - & - & - & - & - & - & - & - & - & - & - & - & - & - & - & + & + \\
\hline Property Sets & $\mathrm{R}$ & & & & & & & & & & & & & & & & & & & & & & \\
\hline $\begin{array}{l}\text { PredefinedType=BATH; } \\
\text { Name=Pset_SanitaryTerminalTypeBath; }\end{array}$ & & & 0 & + & - & - & + & + & + & + & + & + & + & + & + & + & + & + & + & + & + & + & + \\
\hline
\end{tabular}




\begin{tabular}{|c|c|c|c|c|c|c|c|c|c|c|c|c|c|c|c|c|c|c|c|c|c|c|c|}
\hline Entity/Concept & Req & Errors & Count & Flag & 1 & 2 & 3 & 4 & 5 & 6 & 7 & 8 & 9 & $\begin{array}{l}1 \\
0\end{array}$ & $\begin{array}{l}1 \\
1\end{array}$ & $\begin{array}{l}1 \\
2\end{array}$ & $\begin{array}{l}1 \\
3\end{array}$ & $\begin{array}{l}1 \\
4\end{array}$ & $\begin{array}{l}1 \\
5\end{array}$ & $\begin{array}{l}1 \\
6\end{array}$ & $\begin{array}{l}1 \\
7\end{array}$ & $\begin{array}{l}1 \\
8\end{array}$ & $\begin{array}{l}1 \\
9\end{array}$ \\
\hline $\begin{array}{l}\text { PredefinedType=BIDET; } \\
\text { Name=Pset_SanitaryTerminalTypeBidet; } \\
\text { TemplateType= } \\
\text { PSET_TYPEDRIVENOVERRIDE; }\end{array}$ & & & 0 & + & - & - & + & + & + & + & + & + & + & + & + & + & + & + & + & + & + & + & + \\
\hline $\begin{array}{l}\text { PredefinedType=CISTERN; Name= } \\
\text { Pset_SanitaryTerminalTypeCistern; } \\
\text { TemplateType= } \\
\text { PSET_TYPEDRIVENOVERRIDE; }\end{array}$ & & & 0 & + & - & - & + & + & + & + & + & + & + & + & + & + & + & + & + & + & + & + & + \\
\hline $\begin{array}{l}\text { Name= } \\
\text { Pset_SanitaryTerminalTypeCommon; } \\
\text { TemplateType= } \\
\text { PSET_TYPEDRIVENOVERRIDE; }\end{array}$ & & & 0 & + & - & - & + & + & + & + & + & + & + & + & + & + & + & + & + & + & + & + & + \\
\hline $\begin{array}{l}\text { PredefinedType= } \\
\text { SANITARYFOUNTAIN; } \\
\text { Name=Pset_SanitaryTerminalType } \\
\text { SanitaryFountain; TemplateType= } \\
\text { PSET_TYPEDRIVENOVERRIDE; }\end{array}$ & & & 0 & + & - & - & + & + & + & + & + & + & + & + & + & + & + & + & + & + & + & + & + \\
\hline $\begin{array}{l}\text { PredefinedType=SHOWER; Name= } \\
\text { Pset_SanitaryTerminalTypeShower; } \\
\text { TemplateType= } \\
\text { PSET_TYPEDRIVENOVERRIDE; }\end{array}$ & & & 0 & + & - & - & + & + & + & + & + & + & + & + & + & + & + & + & + & + & + & + & + \\
\hline $\begin{array}{l}\text { PredefinedType=SINK; } \\
\text { Name=Pset_SanitaryTerminalTypeSink; } \\
\text { TemplateType= } \\
\text { PSET_TYPEDRIVENOVERRIDE; }\end{array}$ & & & 0 & + & - & - & + & + & + & + & + & + & + & + & + & + & + & + & + & + & + & + & + \\
\hline $\begin{array}{l}\text { PredefinedType=TOILETPAN; Name= } \\
\text { Pset_SanitaryTerminalTypeToiletPan; } \\
\text { TemplateType= } \\
\text { PSET_TYPEDRIVENOVERRIDE; }\end{array}$ & & & 0 & + & - & - & + & + & + & + & + & + & + & + & + & + & + & + & + & + & + & + & + \\
\hline $\begin{array}{l}\text { PredefinedType=URINAL; Name= } \\
\text { Pset_SanitaryTerminalTypeUrinal; } \\
\text { TemplateType= } \\
\text { PSET_TYPEDRIVENOVERRIDE; }\end{array}$ & & & 0 & + & - & - & + & + & + & + & + & + & + & + & + & + & + & + & + & + & + & + & + \\
\hline $\begin{array}{l}\text { PredefinedType=WASHHANDBASIN; } \\
\text { Name=Pset_SanitaryTerminal } \\
\text { TypeWashHandBasin; }\end{array}$ & & & 0 & + & - & - & + & + & + & + & + & + & + & + & + & + & + & + & + & + & + & + & + \\
\hline Quantity Sets & $\mathrm{O}$ & & & & & & & & & & & & & & & & & & & & & & \\
\hline $\begin{array}{l}\text { Name }= \\
\text { Qto_SanitaryTerminalBaseQuantities; }\end{array}$ & & & 0 & + & - & - & - & - & - & - & - & - & - & - & - & - & - & - & - & - & - & - & + \\
\hline Ports [IFC4] & $\mathrm{R}$ & & & & & & & & & & & & & & & & & & & & & & \\
\hline $\begin{array}{l}\text { PredefinedType=BATH; } \\
\text { Name=ColdWater; } \\
\text { Type=DOMESTICCOLDWATER; } \\
\text { Flow=SINK }\end{array}$ & & & 0 & + & - & - & + & + & + & + & + & + & + & + & + & + & + & + & + & + & + & + & + \\
\hline
\end{tabular}




\begin{tabular}{|c|c|c|c|c|c|c|c|c|c|c|c|c|c|c|c|c|c|c|c|c|c|c|c|}
\hline Entity/Concept & Req & Errors & Count & Flag & 1 & 2 & 3 & 4 & 5 & 6 & 7 & 8 & 9 & $\begin{array}{l}1 \\
0\end{array}$ & $\begin{array}{l}1 \\
1\end{array}$ & $\begin{array}{l}1 \\
2\end{array}$ & $\begin{array}{l}1 \\
3\end{array}$ & $\begin{array}{l}1 \\
4\end{array}$ & $\begin{array}{l}1 \\
5\end{array}$ & $\begin{array}{l}1 \\
6\end{array}$ & $\begin{array}{l}1 \\
7\end{array}$ & $\begin{array}{l}1 \\
8\end{array}$ & $\begin{array}{l}1 \\
9\end{array}$ \\
\hline $\begin{array}{l}\text { PredefinedType=BATH; } \\
\text { Name=HotWater; } \\
\text { Type=DOMESTICHOTWATER; } \\
\text { Flow=SINK }\end{array}$ & & & 0 & + & - & - & + & + & + & + & + & + & + & + & + & + & + & + & + & + & + & + & + \\
\hline $\begin{array}{l}\text { PredefinedType=BATH; } \\
\text { Name=Drainage; Type=DRAINAGE; } \\
\text { Flow=SOURCE }\end{array}$ & & & 0 & + & - & - & + & + & + & + & + & + & + & + & + & + & + & + & + & + & + & + & + \\
\hline $\begin{array}{l}\text { PredefinedType=BIDET; } \\
\text { Name=ColdWater;Type= } \\
\text { DOMESTICCOLDWATER; Flow=SINK }\end{array}$ & & & 0 & + & - & - & + & + & + & + & + & + & + & + & + & + & + & + & + & + & + & + & + \\
\hline $\begin{array}{l}\text { PredefinedType=BIDET; } \\
\text { Name=HotWater;Type= } \\
\text { DOMESTICHOTWATER; Flow=SINK }\end{array}$ & & & 0 & + & - & - & + & + & + & + & + & + & + & + & + & + & + & + & + & + & + & + & + \\
\hline $\begin{array}{l}\text { PredefinedType=BIDET; } \\
\text { Name=Drainage; Type=DRAINAGE; } \\
\text { Flow=SOURCE }\end{array}$ & & & 0 & + & - & - & + & + & + & + & + & + & + & + & + & + & + & + & + & + & + & + & + \\
\hline $\begin{array}{l}\text { PredefinedType=CISTERN; } \\
\text { Name=ColdWater;Type= } \\
\text { DOMESTICCOLDWATER; Flow=SINK }\end{array}$ & & & 0 & + & - & - & + & + & + & + & + & + & + & + & + & + & + & + & + & + & + & + & + \\
\hline $\begin{array}{l}\text { PredefinedType=CISTERN; } \\
\text { Name=HotWater;Type= } \\
\text { DOMESTICHOTWATER; Flow=SINK }\end{array}$ & & & 0 & + & - & - & + & + & + & + & + & + & + & + & + & + & + & + & + & + & + & + & + \\
\hline $\begin{array}{l}\text { PredefinedType=CISTERN; } \\
\text { Name=Drainage; Type=DRAINAGE; } \\
\text { Flow=SOURCE }\end{array}$ & & & 0 & + & - & - & + & + & + & + & + & + & + & + & + & + & + & + & + & + & + & + & + \\
\hline $\begin{array}{l}\text { PredefinedType= } \\
\text { SANITARYFOUNTAIN;Name=ColdWater } \\
\text {;Type=DOMESTICCOLDWATER; } \\
\text { Flow=SINK }\end{array}$ & & & 0 & + & - & - & + & + & + & + & + & + & + & + & + & + & + & + & + & + & + & + & + \\
\hline $\begin{array}{l}\text { PredefinedType= } \\
\text { SANITARYFOUNTAIN;Name=HotWater; } \\
\text { Type=DOMESTICHOTWATER; } \\
\text { Flow=SINK }\end{array}$ & & & 0 & + & - & - & + & + & + & + & + & + & + & + & + & + & + & + & + & + & + & + & + \\
\hline $\begin{array}{l}\text { PredefinedType= } \\
\text { SANITARYFOUNTAIN; Name=Drainage; } \\
\text { Type=DRAINAGE; Flow=SOURCE }\end{array}$ & & & 0 & + & - & - & + & + & + & + & + & + & + & + & + & + & + & + & + & + & + & + & + \\
\hline $\begin{array}{l}\text { PredefinedType=SHOWER; } \\
\text { Name=ColdWater;Type= } \\
\text { DOMESTICCOLDWATER; Flow=SINK }\end{array}$ & & & 0 & + & - & - & + & + & + & + & + & + & + & + & + & + & + & + & + & + & + & + & + \\
\hline $\begin{array}{l}\text { PredefinedType=SHOWER; } \\
\text { Name=HotWater;Type= } \\
\text { DOMESTICHOTWATER; Flow=SINK }\end{array}$ & & & 0 & + & - & - & + & + & + & + & + & + & + & + & + & + & + & + & + & + & + & + & + \\
\hline $\begin{array}{l}\text { PredefinedType=SHOWER; } \\
\text { Name=Drainage; Type=DRAINAGE; } \\
\text { Flow=SOURCE }\end{array}$ & & & 0 & + & - & - & + & + & + & + & + & + & + & + & + & + & + & + & + & + & + & + & + \\
\hline
\end{tabular}


PredefinedType=SINK;Name $=$

ColdWater;Type=

DOMESTICCOLDWATER; Flow=SINK

PredefinedType $=$ SINK; Name $=$

HotWater;Type=
DOMESTICHOTWATER; Flow=SINK

PredefinedType=SINK; Name=Drainage;

Type=DRAINAGE; Flow=SOURCE

PredefinedType=TOILETPAN;

Name $=$ ColdWater;Type $=$

DOMESTICCOLDWATER; Flow=SINK

PredefinedType=TOILETPAN;

Name=Drainage; Type=DRAINAGE;

Flow=SOURCE

PredefinedType=URINAL;

Name $=$ ColdWater;Type $=$

DOMESTICCOLDWATER; Flow=SINK

PredefinedType=URINAL;

Name=Drainage; Type=DRAINAGE;

Flow=SOURCE

PredefinedType $=$ WASHHANDBASIN;

Name $=$ ColdWater; Type $=$

DOMESTICCOLDWATER; Flow=SINK

PredefinedType $=$ WASHHANDBASIN;

Name=HotWater;Type $=$

DOMESTICHOTWATER; Flow=SINK

PredefinedType $=$ WASHHANDBASIN;

Name=Drainage; Type=DRAINAGE;

Flow=SOURCE

Product Placement

Type=IfcLocalPlacement;

Footprint Geometry

RepresentationType=Geometric CurveSe

- Geometry=IfcGeometricCurveSet;

Body Geometry

RepresentationType $=$

MappedRepresentation;Geomtry=

IfcMappedltem;

Clearance Geometry

\section{\begin{tabular}{llllllllll}
\hline & 2 & 2 & 3 & 4 & 5 & 6 & 7 & 8 & 9 \\
\hline
\end{tabular}}

\begin{tabular}{|c|c|c|c|c|c|c|c|c|c|c|c|c|c|c|c|c|c|c|c|c|c|}
\hline & 0 & + & - & - & + & + & + & + & + & + & + & + & + & + & + & + & + & + & + & + & + \\
\hline & 0 & + & - & - & + & + & + & + & + & + & + & + & + & + & + & + & + & + & + & + & + \\
\hline & 0 & + & - & - & + & + & + & + & + & + & + & + & + & + & + & + & + & + & + & + & + \\
\hline & 0 & + & - & - & + & + & + & + & + & + & + & + & + & + & + & + & + & + & + & + & + \\
\hline & 0 & + & - & - & + & + & + & + & + & + & + & + & + & + & + & + & + & + & + & + & + \\
\hline $\mathrm{R}$ & & & & & & & & & & & & & & & & & & & & & \\
\hline & 0 & + & - & - & + & + & + & + & + & + & + & + & + & + & + & + & + & + & + & + & + \\
\hline $\mathrm{R}$ & & & & & & & & & & & & & & & & & & & & & \\
\hline & 0 & + & $=$ & - & + & - & - & - & - & - & - & + & + & + & + & + & + & + & + & + & + \\
\hline $\mathrm{R}$ & & & & & & & & & & & & & & & & & & & & & \\
\hline & 0 & + & - & - & + & - & - & - & - & - & - & + & + & + & + & + & + & + & + & + & + \\
\hline 0 & 0 & + & - & - & + & - & - & - & - & - & - & + & + & + & + & + & + & + & + & + & + \\
\hline
\end{tabular}




\begin{tabular}{|c|c|c|c|c|c|c|c|c|c|c|c|c|c|c|c|c|c|c|c|c|c|c|c|}
\hline Entity/Concept & Req & Errors & Count & Flag & 1 & 2 & 3 & 4 & 5 & 6 & 7 & 8 & 9 & $\begin{array}{l}1 \\
0\end{array}$ & $\begin{array}{l}1 \\
1\end{array}$ & $\begin{array}{l}1 \\
2\end{array}$ & $\begin{array}{l}1 \\
3\end{array}$ & $\begin{array}{l}1 \\
4\end{array}$ & $\begin{array}{l}1 \\
5\end{array}$ & $\begin{array}{l}1 \\
6\end{array}$ & $\begin{array}{l}1 \\
7\end{array}$ & $\begin{array}{l}1 \\
8\end{array}$ & $\begin{array}{l}1 \\
9\end{array}$ \\
\hline Element Connectivity & $\mathrm{O}$ & & & & & & & & & & & & & & & & & & & & & & \\
\hline Type=IfcSlab; & & & 0 & + & - & - & - & - & - & - & - & - & - & + & + & + & + & + & + & + & + & + & + \\
\hline Type=IfcCovering; & & & 0 & + & - & - & - & - & - & - & - & - & - & + & + & + & + & + & + & + & + & + & + \\
\hline Type=IfcSystemFurnitureElement; & & & 0 & + & - & - & - & - & - & - & - & - & - & + & + & + & + & + & + & + & + & + & + \\
\hline IfcSanitaryTerminalType & & & 9 & & & & & & & & & & & & & & & & & & & & \\
\hline Identity & $\mathrm{R}$ & & 9 & + & - & - & - & - & - & - & - & - & - & - & - & - & - & - & - & - & - & + & + \\
\hline Property Sets for Types & $\mathrm{R}$ & & & & & & & & & & & & & & & & & & & & & & \\
\hline Name= Pset_SanitaryTerminalCommon; & & $\begin{array}{l}\# 1794 \\
8\end{array}$ & 0 & $\mathbf{F}$ & - & - & - & - & - & - & - & - & - & - & - & - & - & - & - & - & - & $\mathbf{F}$ & $\mathbf{F}$ \\
\hline Type-Based Ports [IFC4] & $\mathrm{R}$ & $\begin{array}{l}\# 1794 \\
8 \\
\end{array}$ & 0 & $F$ & - & - & - & - & - & - & - & - & - & - & - & - & - & - & - & - & - & $\mathbf{F}$ & $\mathbf{F}$ \\
\hline Body Geometry & $\mathrm{R}$ & & & & & & & & & & & & & & & & & & & & & & \\
\hline $\begin{array}{l}\text { RepresentationType=Brep; } \\
\text { Geomtry=IfcFacetedBrep; }\end{array}$ & & & 9 & + & - & - & - & - & - & - & - & - & - & - & - & - & - & - & - & - & - & + & + \\
\hline Clearance Geometry & $\mathrm{R}$ & & & & & & & & & & & & & & & & & & & & & & \\
\hline $\begin{array}{l}\text { Type=SurfaceModel; } \\
\text { Geomtry=IfcFaceBasedSurfaceModel; }\end{array}$ & & & 9 & + & - & - & - & - & - & - & - & - & - & - & - & - & - & - & - & - & - & + & + \\
\hline IfcWasteTerminal & & & 0 & & & & & & & & & & & & & & & & & & & & \\
\hline Identity & $\mathrm{R}$ & & 0 & + & - & - & - & - & - & - & - & - & - & + & + & + & + & + & + & + & + & + & + \\
\hline Object Typing & $\mathrm{R}$ & & & & & & & & & & & & & & & & & & & & & & \\
\hline Type=IfcWasteTerminalType; & & & 0 & + & - & - & - & - & - & - & - & - & - & - & - & - & - & - & - & - & - & + & + \\
\hline Property Sets & $\mathrm{R}$ & & & & & & & & & & & & & & & & & & & & & & \\
\hline $\begin{array}{l}\text { PredefinedType }=; \text { Name }= \\
\text { Pset_WasteTerminalTypeCommon; }\end{array}$ & & & 0 & + & - & - & - & - & - & - & - & - & - & + & + & + & + & + & + & + & + & + & + \\
\hline $\begin{array}{l}\text { PredefinedType=FLOORTRAP; } \\
\text { Name=Pset_WasteTerminalTypeFloorTr } \\
\text { ap;TemplateType= } \\
\text { PSET_TYPEDRIVENOVERRIDE; }\end{array}$ & & & 0 & + & - & - & - & - & - & - & - & - & - & + & + & + & + & + & + & + & + & + & + \\
\hline $\begin{array}{l}\text { PredefinedType=FLOORWASTE; } \\
\text { Name= } \\
\text { Pset_WasteTerminalTypeFloorWaste; } \\
\text { TemplateType= } \\
\text { PSET_TYPEDRIVENOVERRIDE; }\end{array}$ & & & 0 & + & - & - & - & - & - & - & - & - & - & + & + & + & + & + & + & + & + & + & + \\
\hline
\end{tabular}




\section{Entity/Concept}

$\begin{array}{lllllllllllll}\text { Req Errors } & \text { Count } & \text { Flag } & 1 & 2 & 3 & 4 & 5 & 6 & 7 & 8 & 9\end{array}$

PredefinedType=GULLYSUMP; Name Pset_WasteTerminalTypeGullySump TemplateType $=$

PSET_TYPEDRIVENOVERRIDE;

PredefinedType=GULLYTRAP; Name= Pset_WasteTerminalTypeGullyTrap;

TemplateType $=$

PSET TYPEDRIVENOVERRIDE:

PredefinedType=ROOFDRAIN; Name= Pset_WasteTerminalTypeRoofDrain;

TemplateType $=$

PSET_TYPEDRIVENOVERRIDE;

\section{PredefinedType $=$}

WASTEDISPOSALUNIT;Name=

Pset_WasteTerminalTypeWaste

DisposalUnit;TemplateType=

PSET_TYPEDRIVENOVERRIDE;

PredefinedType=WASTETRAP; Name

Pset_WasteTerminalTypeWasteTrap;

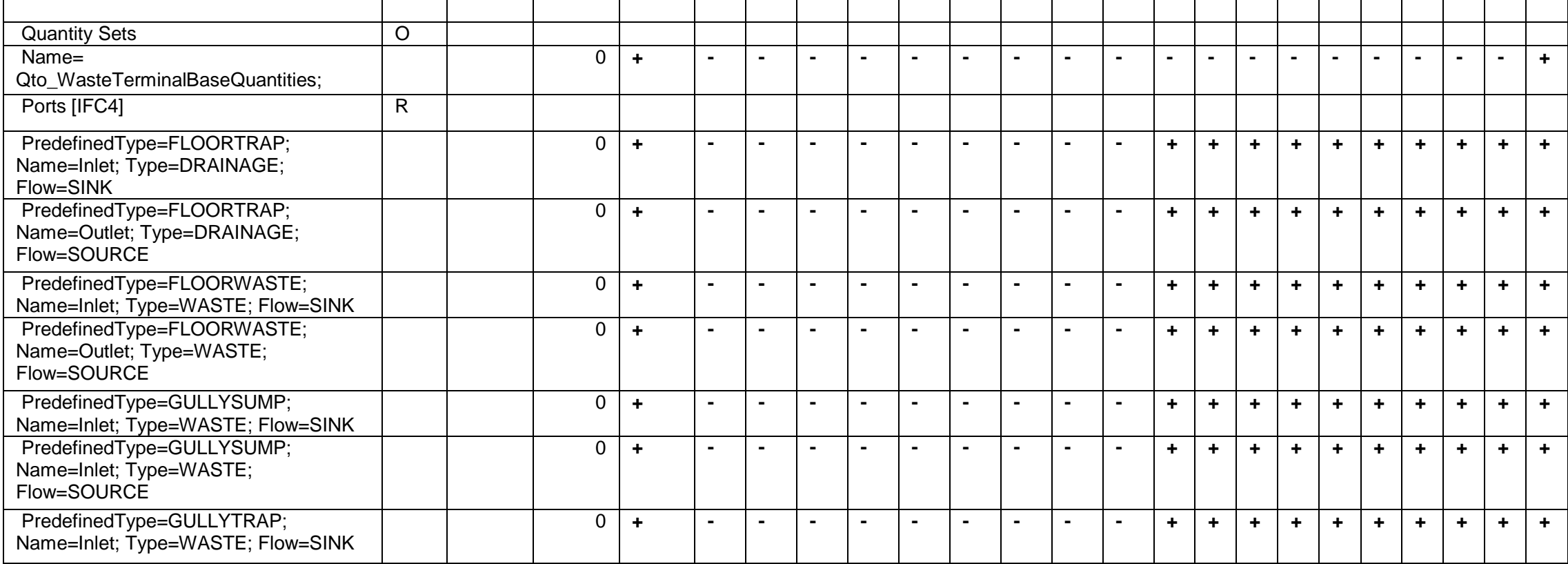

Ports [IFC4]

PredefinedType $=$ FLOO

Flow=SINK

PredefinedType=FLOORTRAP;

Prome=Outlet; Type=DRAINAGE;

Flow=SOURCE

Flow=SOURCE

PredefinedType $=$ GULLYTRAP

Name=Inlet; Type=WASTE; Flow=SINK 


\begin{tabular}{|c|c|c|c|c|c|c|c|c|c|c|c|c|c|c|c|c|c|c|c|c|c|c|c|}
\hline Entity/Concept & Req & Errors & Count & Flag & 1 & 2 & 3 & 4 & 5 & 6 & 7 & 8 & 9 & $\begin{array}{l}1 \\
0\end{array}$ & $\begin{array}{l}1 \\
1\end{array}$ & $\begin{array}{l}1 \\
2\end{array}$ & $\begin{array}{l}1 \\
3\end{array}$ & $\begin{array}{l}1 \\
4\end{array}$ & $\begin{array}{l}1 \\
5\end{array}$ & $\begin{array}{l}1 \\
6\end{array}$ & 7 & & $\begin{array}{l}1 \\
9\end{array}$ \\
\hline $\begin{array}{l}\text { PredefinedType=GULLYTRAP; } \\
\text { Name=Inlet; Type=WASTE; } \\
\text { Flow=SOURCE }\end{array}$ & & & 0 & + & - & - & - & - & - & - & - & - & - & + & + & + & + & + & + & + & + & & + \\
\hline $\begin{array}{l}\text { PredefinedType=ROOFDRAIN; } \\
\text { Name=Outlet; Type=RAINWATER; } \\
\text { Flow=SOURCE }\end{array}$ & & & 0 & + & - & - & - & - & - & - & - & - & - & + & + & + & + & + & + & + & + & & + \\
\hline $\begin{array}{l}\text { PredefinedType=WASTEDISPOSALUNI } \\
\text { T; Name=Inlet; Type=WASTE; } \\
\text { Flow=SINK }\end{array}$ & & & 0 & + & - & - & - & - & - & - & - & - & - & + & + & + & + & + & + & + & + & & + \\
\hline $\begin{array}{l}\text { PredefinedType= } \\
\text { WASTEDISPOSALUNIT; Name=Outlet; } \\
\text { Type=WASTE; Flow=SOURCE }\end{array}$ & & & 0 & + & - & - & - & - & - & - & - & - & - & + & + & + & + & + & + & + & + & & + \\
\hline $\begin{array}{l}\text { PredefinedType=WASTETRAP; } \\
\text { Name=Inlet; Type=WASTE; Flow=SINK }\end{array}$ & & & 0 & + & - & - & - & - & - & - & - & - & - & + & + & + & + & + & + & + & + & & + \\
\hline $\begin{array}{l}\text { PredefinedType=WASTETRAP; } \\
\text { Name=Outlet; Type=WASTE; } \\
\text { Flow=SOURCE }\end{array}$ & & & 0 & + & - & - & - & - & - & - & - & - & - & + & + & + & + & + & + & + & + & & + \\
\hline Body Geometry & $\mathrm{R}$ & & & & & & & & & & & & & & & & & & & & & & \\
\hline $\begin{array}{l}\text { RepresentationType= } \\
\text { MappedRepresentation; } \\
\text { Geomtry=IfcMappedItem; }\end{array}$ & & & 0 & + & - & - & - & - & - & - & - & - & - & + & + & + & + & + & + & + & + & & + \\
\hline Clearance Geometry & $\mathrm{O}$ & & 0 & + & - & - & - & - & - & - & - & - & - & + & + & + & + & + & + & + & + & & + \\
\hline IfcPipeFitting & & & 0 & & & & & & & & & & & & & & & & & & & & \\
\hline Identity & $\mathrm{R}$ & & 0 & + & - & - & - & - & - & - & - & - & + & + & + & + & + & + & + & + & + & & + \\
\hline Object Typing & $\mathrm{R}$ & & & & & & & & & & & & & & & & & & & & & & \\
\hline Type=IfcPipeFittingType; & & & 0 & + & - & - & - & - & - & - & - & - & - & - & - & - & - & - & - & - & - & & + \\
\hline Property Sets & $\mathrm{R}$ & & & & & & & & & & & & & & & & & & & & & & \\
\hline $\begin{array}{l}\text { PredefinedType=; } \\
\text { Name=Pset_PipeFittingOccurrence; }\end{array}$ & & & 0 & + & - & - & - & - & - & - & - & - & - & - & - & - & - & - & + & + & + & & + \\
\hline $\begin{array}{l}\text { Name=Pset_PipeFittingPHistory; } \\
\text { TemplateType= } \\
\text { PSET_PERFORMANCEDRIVEN; }\end{array}$ & & & 0 & + & - & - & - & - & - & - & - & - & - & - & - & - & - & - & + & + & + & & + \\
\hline $\begin{array}{l}\text { PredefinedType=BEND; } \\
\text { Name=Pset_PipeFittingTypeBend; } \\
\text { TemplateType= } \\
\text { PSET_TYPEDRIVENOVERRIDE; }\end{array}$ & & & 0 & + & - & - & - & - & - & - & - & - & - & - & - & - & - & - & + & + & + & & + \\
\hline $\begin{array}{l}\text { Name=Pset_PipeFittingTypeCommon; } \\
\text { TemplateType= } \\
\text { PSET_TYPEDRIVENOVERRIDE; }\end{array}$ & & & 0 & + & - & - & - & - & - & - & - & - & - & - & - & - & - & - & + & + & + & & + \\
\hline
\end{tabular}




\begin{tabular}{|c|c|c|c|c|c|c|c|c|c|c|c|c|c|c|c|c|c|c|c|c|c|c|c|}
\hline Entity/Concept & Req & Errors & Count & Flag & 1 & 2 & 3 & 4 & 5 & 6 & 7 & 8 & 9 & $\begin{array}{l}1 \\
0\end{array}$ & $\begin{array}{l}1 \\
1\end{array}$ & $\begin{array}{l}1 \\
2\end{array}$ & $\begin{array}{l}1 \\
3\end{array}$ & $\begin{array}{l}1 \\
4\end{array}$ & $\begin{array}{l}1 \\
5\end{array}$ & $\begin{array}{l}1 \\
6\end{array}$ & $\begin{array}{l}1 \\
7\end{array}$ & $\begin{array}{l}1 \\
8\end{array}$ & $\begin{array}{l}1 \\
9\end{array}$ \\
\hline $\begin{array}{l}\text { PredefinedType=JUNCTION; } \\
\text { Name=Pset_PipeFittingTypeJunction; }\end{array}$ & & & 0 & + & - & - & - & - & - & - & - & - & - & - & - & - & - & - & + & + & + & + & + \\
\hline Quantity Sets & $\mathrm{R}$ & & & & & & & & & & & & & & & & & & & & & & \\
\hline Name=Qto_PipeFittingBaseQuantities; & & & 0 & + & - & - & - & - & - & - & - & - & - & - & - & - & - & - & - & - & - & - & + \\
\hline Ports [IFC4] & $\mathrm{R}$ & & & & & & & & & & & & & & & & & & & & & & \\
\hline $\begin{array}{l}\text { PredefinedType=BEND; Name=Inlet; } \\
\text { Type=NOTDEFINED; Flow=SINK }\end{array}$ & & & 0 & + & - & - & - & - & - & - & - & - & - & - & - & - & - & - & + & + & + & + & + \\
\hline $\begin{array}{l}\text { PredefinedType=BEND; Name=Outlet; } \\
\text { Type=NOTDEFINED; Flow=SOURCE }\end{array}$ & & & 0 & + & - & - & - & - & - & - & - & - & - & - & - & - & - & - & + & + & + & + & + \\
\hline $\begin{array}{l}\text { PredefinedType=CONNECTOR; } \\
\text { Name=Inlet; Type=NOTDEFINED; } \\
\text { Flow=SINK }\end{array}$ & & & 0 & + & - & - & - & - & - & - & - & - & - & - & - & - & - & - & + & + & + & + & + \\
\hline $\begin{array}{l}\text { PredefinedType=CONNECTOR; } \\
\text { Name=Outlet; Type=NOTDEFINED; } \\
\text { Flow=SOURCE }\end{array}$ & & & 0 & + & - & - & - & - & - & - & - & - & - & - & - & - & - & - & + & + & + & + & + \\
\hline $\begin{array}{l}\text { PredefinedType=ENTRY; Name=Outlet; } \\
\text { Type=NOTDEFINED; Flow=SOURCE }\end{array}$ & & & 0 & + & - & - & - & - & - & - & - & - & - & - & - & - & - & - & + & + & + & + & + \\
\hline $\begin{array}{l}\text { PredefinedType=EXIT; Name=Inlet; } \\
\text { Type=NOTDEFINED; Flow=SINK }\end{array}$ & & & 0 & + & - & - & - & - & - & - & - & - & - & - & - & - & - & - & + & + & + & + & + \\
\hline $\begin{array}{l}\text { PredefinedType=JUNCTION; } \\
\text { Name=Inlet; Type=NOTDEFINED; } \\
\text { Flow=SINK }\end{array}$ & & & 0 & + & - & - & - & - & - & - & - & - & - & - & - & - & - & - & + & + & + & + & + \\
\hline $\begin{array}{l}\text { PredefinedType=JUNCTION; } \\
\text { Name=Outlet\#1; Type=NOTDEFINED; } \\
\text { Flow=SOURCE }\end{array}$ & & & 0 & + & - & - & - & - & - & - & - & - & - & - & - & - & - & - & + & + & + & + & + \\
\hline $\begin{array}{l}\text { PredefinedType=JUNCTION; } \\
\text { Name=Outlet\#2; Type=NOTDEFINED; } \\
\text { Flow=SOURCE }\end{array}$ & & & 0 & + & - & - & - & - & - & - & - & - & - & - & - & - & - & - & + & + & + & + & + \\
\hline $\begin{array}{l}\text { PredefinedType=OBSTRUCTION; } \\
\text { Name=Inlet; Type=NOTDEFINED; } \\
\text { Flow=SINK }\end{array}$ & & & 0 & + & - & - & - & - & - & - & - & - & - & - & - & - & - & - & + & + & + & + & + \\
\hline $\begin{array}{l}\text { PredefinedType=OBSTRUCTION; } \\
\text { Name=Outlet; Type=NOTDEFINED; } \\
\text { Flow=SOURCE }\end{array}$ & & & 0 & + & - & - & - & - & - & - & - & - & - & - & - & - & - & - & + & + & + & + & + \\
\hline IfcPipeFittingType & & & 372 & & & & & & & & & & & & & & & & & & & & \\
\hline Identity & $\mathrm{R}$ & & 372 & + & - & - & - & - & - & - & - & - & - & - & - & - & - & - & - & - & - & + & + \\
\hline Property Sets for Types & $\mathrm{R}$ & \#3783 & 0 & $\mathbf{F}$ & - & - & - & - & - & - & - & - & - & - & - & - & - & - & - & - & - & $F$ & $F$ \\
\hline IfcPipeSegment & & & 0 & & & & & & & & & & & & & & & & & & & & \\
\hline Identity & $\mathrm{R}$ & & 0 & + & - & - & - & - & - & - & - & - & - & + & + & + & + & + & + & + & + & + & + \\
\hline
\end{tabular}




\begin{tabular}{|c|c|c|c|c|c|c|c|c|c|c|c|c|c|c|c|c|c|c|c|c|c|c|c|c|}
\hline Entity/Concept & Req & Errors & Count & Flag & 1 & 2 & 3 & 4 & 5 & 6 & 7 & 8 & 9 & $\begin{array}{l}1 \\
0\end{array}$ & $\begin{array}{l}1 \\
1\end{array}$ & $\begin{array}{l}1 \\
2\end{array}$ & $\begin{array}{l}1 \\
3\end{array}$ & $\begin{array}{l}1 \\
4\end{array}$ & $\begin{array}{l}1 \\
5\end{array}$ & $\begin{array}{l}1 \\
6\end{array}$ & $\begin{array}{l}1 \\
7\end{array}$ & & & $\begin{array}{l}1 \\
9\end{array}$ \\
\hline Object Typing & $\mathrm{R}$ & & & & & & & & & & & & & & & & & & & & & & & \\
\hline Type=IfcPipeSegmentType; & & & 0 & + & - & - & - & - & - & - & - & - & - & - & - & - & - & - & - & - & - & & & + \\
\hline Property Sets & $\mathrm{R}$ & & & & & & & & & & & & & & & & & & & & & & & \\
\hline $\begin{array}{l}\text { PredefinedType=; } \\
\text { Name=Pset_PipeConnectionFlanged; }\end{array}$ & & & 0 & + & - & - & - & - & - & - & - & - & - & - & - & - & - & - & + & + & + & & & + \\
\hline $\begin{array}{l}\text { Name=Pset_PipeSegmentOccurrence; } \\
\text { TemplateType=PSET_OCCURRENCED } \\
\text { RIVEN; }\end{array}$ & & & 0 & + & - & - & - & - & - & - & - & - & - & - & - & - & - & - & + & + & + & & & + \\
\hline $\begin{array}{l}\text { Name=Pset_PipeSegmentPHistory; } \\
\text { TemplateType= } \\
\text { PSET_PERFORMANCEDRIVEN; }\end{array}$ & & & 0 & + & - & - & - & - & - & - & - & - & - & - & - & - & - & - & + & + & + & + & & + \\
\hline $\begin{array}{l}\text { Name= } \\
\text { Pset_PipeSegmentTypeCommon; } \\
\text { TemplateType= } \\
\text { PSET_TYPEDRIVENOVERRIDE; }\end{array}$ & & & 0 & + & - & - & - & - & - & - & - & - & - & - & - & - & - & - & + & + & + & & & + \\
\hline $\begin{array}{l}\text { PredefinedType=CULVERT; } \\
\text { Name=Pset_PipeSegmentTypeCulvert; } \\
\text { TemplateType= } \\
\text { PSET_TYPEDRIVENOVERRIDE; }\end{array}$ & & & 0 & + & - & - & - & - & - & - & - & - & - & - & - & - & - & - & + & + & + & & & + \\
\hline $\begin{array}{l}\text { PredefinedType=GUTTER; } \\
\text { Name=Pset_PipeSegmentTypeGutter; }\end{array}$ & & & 0 & + & - & - & - & - & - & - & - & - & - & - & - & - & - & - & + & + & + & & & + \\
\hline Quantity Sets & $\mathrm{R}$ & & & & & & & & & & & & & & & & & & & & & & & \\
\hline $\begin{array}{l}\text { Name= } \\
\text { Qto_PipeSegmentBaseQuantities; }\end{array}$ & & & 0 & + & - & - & - & - & - & - & - & - & - & - & - & - & - & - & - & - & - & - & & + \\
\hline Material Profile Set Usage [IFC4] & $\mathrm{R}$ & & & & & & & & & & & & & & & & & & & & & & & \\
\hline Name=Casing; & & & 0 & + & - & - & - & - & - & - & - & - & + & + & + & + & + & + & + & + & + & & & + \\
\hline Name=Coating; & & & 0 & + & - & - & - & - & - & - & - & - & + & + & + & + & + & + & + & + & + & & & + \\
\hline Name=Insulation; & & & 0 & + & - & - & - & - & - & - & - & - & + & + & + & + & + & + & + & + & + & & & + \\
\hline Name=Lining; & & & 0 & + & - & - & - & - & - & - & - & - & + & + & + & + & + & + & + & + & + & & & + \\
\hline Ports [IFC4] & $\mathrm{R}$ & & & & & & & & & & & & & & & & & & & & & & & \\
\hline $\begin{array}{l}\text { Name=Inlet; Type=NOTDEFINED; } \\
\text { Flow=SINK }\end{array}$ & & & 0 & + & - & - & - & - & - & - & - & - & + & + & + & + & + & + & + & + & + & & & + \\
\hline $\begin{array}{l}\text { Name=Outlet; Type=NOTDEFINED; } \\
\text { Flow=SOURCE }\end{array}$ & & & 0 & + & - & - & - & - & - & - & - & - & + & + & + & + & + & + & + & + & + & & & + \\
\hline Interference & $\mathrm{R}$ & & 0 & + & - & - & - & - & - & - & - & - & - & + & + & + & + & + & + & + & + & & & + \\
\hline Axis Geometry & $\mathrm{R}$ & & & & & & & & & & & & & & & & & & & & & & & \\
\hline
\end{tabular}




\begin{tabular}{|c|c|c|c|c|c|c|c|c|c|c|c|c|c|c|c|c|c|c|c|c|c|c|c|}
\hline EntitylConcept & Req & Errors & Count & Flag & 1 & 2 & 3 & 4 & 5 & 6 & 7 & 8 & 9 & $\begin{array}{l}1 \\
0\end{array}$ & $\begin{array}{l}1 \\
1\end{array}$ & $\begin{array}{l}1 \\
2\end{array}$ & $\begin{array}{l}1 \\
3\end{array}$ & $\begin{array}{l}1 \\
4\end{array}$ & $\begin{array}{l}1 \\
5\end{array}$ & $\begin{array}{l}1 \\
6\end{array}$ & $\begin{array}{l}1 \\
7\end{array}$ & $\begin{array}{l}1 \\
8\end{array}$ & $\begin{array}{l}1 \\
9\end{array}$ \\
\hline $\begin{array}{l}\text { RepresentationType=Curve3D; Geome- } \\
\text { try=IfcPolyline; }\end{array}$ & & & 0 & + & - & - & - & - & - & - & - & - & - & + & + & + & + & + & + & + & + & + & + \\
\hline Body Geometry & $\mathrm{R}$ & & & & & & & & & & & & & & & & & & & & & & \\
\hline $\begin{array}{l}\text { RepresentationType= } \\
\text { MappedRepresentation; Geometry= } \\
\text { IfcMappedltem; }\end{array}$ & & & 0 & + & - & - & - & - & - & - & - & - & - & - & - & - & - & - & - & + & + & + & + \\
\hline $\begin{array}{l}\text { RepresentationType=SweptSolid; Ge- } \\
\text { ometry=IfcExtrudedAreaSolid; }\end{array}$ & & & 0 & + & - & - & - & - & - & - & - & - & - & - & - & - & - & - & - & + & + & + & + \\
\hline IfcPipeSegmentType & & & 6 & & & & & & & & & & & & & & & & & & & & \\
\hline Identity & $\mathrm{R}$ & & 6 & + & - & - & - & - & - & - & - & - & - & - & - & - & - & - & - & - & - & + & + \\
\hline Property Sets for Types & $\mathrm{R}$ & $\# 825$ & 0 & $F$ & - & - & - & - & - & - & - & - & - & - & - & - & - & - & - & - & - & $\mathrm{F}$ & $\mathbf{F}$ \\
\hline Material Profile Set [IFC4] & $\mathrm{R}$ & $\# 825$ & 0 & $\mathbf{F}$ & - & - & - & - & - & - & - & - & - & - & - & - & - & - & - & - & - & $F$ & $F$ \\
\hline IfcPump & & & 0 & & & & & & & & & & & & & & & & & & & & \\
\hline Identity & $\mathrm{R}$ & & 0 & + & - & - & - & + & + & + & + & + & + & + & + & + & + & + & + & + & + & + & + \\
\hline Object Typing & $\mathrm{R}$ & & & & & & & & & & & & & & & & & & & & & & \\
\hline Type=IfcPumpType; & & & 0 & + & - & - & - & - & - & - & - & - & - & - & - & - & - & - & - & - & - & + & + \\
\hline Property Sets & $\mathrm{R}$ & & 0 & + & - & - & - & + & + & + & + & + & + & + & + & + & + & + & + & + & + & + & + \\
\hline Quantity Sets & $\mathrm{O}$ & & & & & & & & & & & & & & & & & & & & & & \\
\hline Name=Qto_PumpBaseQuantities; & & & 0 & + & - & - & - & - & - & - & - & - & - & - & - & - & - & - & - & - & - & - & + \\
\hline Ports [IFC4] & $\mathrm{R}$ & & & & & & & & & & & & & & & & & & & & & & \\
\hline $\begin{array}{l}\text { Name=Power; Type=ELECTRICAL; } \\
\text { Flow=SINK }\end{array}$ & & & 0 & + & - & - & - & + & + & + & + & + & + & + & + & + & + & + & + & + & + & + & + \\
\hline $\begin{array}{l}\text { Name=Inlet; Type=NOTDEFINED; } \\
\text { Flow=SINK }\end{array}$ & & & 0 & + & - & - & - & + & + & + & + & + & + & + & + & + & + & + & + & + & + & + & + \\
\hline $\begin{array}{l}\text { Name=Outlet; Type=NOTDEFINED; } \\
\text { Flow=SOURCE }\end{array}$ & & & 0 & + & - & - & - & + & + & + & + & + & + & + & + & + & + & + & + & + & + & + & + \\
\hline Spatial Containment & $\mathrm{R}$ & & 0 & + & - & - & - & - & - & + & + & + & + & + & + & + & + & + & + & + & + & + & + \\
\hline Body Geometry & $\mathrm{R}$ & & & & & & & & & & & & & & & & & & & & & & \\
\hline $\begin{array}{l}\text { RepresentationType= } \\
\text { MappedRepresentation; Geometry= } \\
\text { IfcMappedltem; }\end{array}$ & & & 0 & + & - & - & - & - & - & - & - & - & - & + & + & + & + & + & + & + & + & + & + \\
\hline IfcPumpTyре & & & 1 & & & & & & & & & & & & & & & & & & & & \\
\hline Identity & $\mathrm{R}$ & & 1 & + & - & - & - & - & - & - & - & - & - & - & - & - & - & - & - & - & - & + & + \\
\hline Property Sets for Types & $\mathrm{R}$ & $\begin{array}{l}\# 1036 \\
542\end{array}$ & 0 & $\mathbf{F}$ & - & - & - & - & - & - & - & - & - & - & - & - & - & - & - & - & - & $F$ & $\mathbf{F}$ \\
\hline
\end{tabular}




\begin{tabular}{|c|c|c|c|c|c|c|c|c|c|c|c|c|c|c|c|c|c|c|c|c|c|c|c|}
\hline Entity/Concept & Req & Errors & Count & Flag & 1 & 2 & 3 & 4 & 5 & 6 & 7 & 8 & 9 & $\begin{array}{l}1 \\
0\end{array}$ & $\begin{array}{l}1 \\
1\end{array}$ & $\begin{array}{l}1 \\
2\end{array}$ & $\begin{array}{l}1 \\
3\end{array}$ & $\begin{array}{l}1 \\
4\end{array}$ & $\begin{array}{l}1 \\
5\end{array}$ & $\begin{array}{l}1 \\
6\end{array}$ & $\begin{array}{l}1 \\
7\end{array}$ & $\begin{array}{l}1 \\
8\end{array}$ & $\begin{array}{l}1 \\
9\end{array}$ \\
\hline Type-Based Ports [IFC4] & $\mathrm{R}$ & $\begin{array}{l}\# 1036 \\
542\end{array}$ & 0 & $\mathbf{F}$ & - & - & - & - & - & - & - & - & - & - & - & - & - & - & - & - & - & $\mathbf{F}$ & $\mathbf{F}$ \\
\hline IfcDistributionPort & & & 13058 & & & & & & & & & & & & & & & & & & & & \\
\hline Identity & $\mathrm{R}$ & & 13058 & + & - & - & + & + & + & + & + & + & + & + & + & + & + & + & + & + & + & + & + \\
\hline Property Sets & $\mathrm{R}$ & & & & & & & & & & & & & & & & & & & & & & \\
\hline $\begin{array}{l}\text { PredefinedType=PIPE; } \\
\text { Name=Pset_DistributionPortTypePipe; }\end{array}$ & & $\begin{array}{l}\# 2415 \\
657\end{array}$ & 0 & $\mathbf{F}$ & - & - & * & * & * & * & * & $\mathbf{F}$ & * & * & $*$ & * & $\mathbf{F}$ & $\mathbf{F}$ & $\mathbf{F}$ & $\mathbf{F}$ & $\mathbf{F}$ & $\mathbf{F}$ & $\mathbf{F}$ \\
\hline Material Profile Set Usage [IFC4] & $\mathrm{R}$ & & & & & & & & & & & & & & & & & & & & & & \\
\hline $\begin{array}{l}\text { Name=Pipe; Pro- } \\
\text { file=IfcCircleHollowProfileDef; }\end{array}$ & & $\begin{array}{l}\# 2415 \\
657\end{array}$ & 0 & $F$ & - & - & - & - & - & - & - & - & - & $F$ & $F$ & $F$ & $F$ & $F$ & $F$ & $F$ & $F$ & $F$ & $F$ \\
\hline Port Connectivity & $\mathrm{R}$ & $\begin{array}{l}\# 2415 \\
659 \\
\end{array}$ & 6529 & $\mathbf{F}$ & - & - & - & - & - & - & - & - & - & $\mathbf{F}$ & $\mathbf{F}$ & $\mathbf{F}$ & $\mathbf{F}$ & $\mathbf{F}$ & $\mathbf{F}$ & $\mathbf{F}$ & $\mathbf{F}$ & $\mathbf{F}$ & $\mathbf{F}$ \\
\hline Product Placement & $\mathrm{R}$ & & 13058 & + & - & - & + & - & - & - & - & - & - & - & - & - & - & - & + & + & + & + & + \\
\hline IfcDistributionSystem & & & 0 & & & & & & & & & & & & & & & & & & & & \\
\hline Identity & $\mathrm{R}$ & & 0 & + & - & + & - & - & - & - & - & - & - & - & - & - & - & - & - & - & - & - & - \\
\hline Property Sets & $\mathrm{R}$ & & & & & & & & & & & & & & & & & & & & & & \\
\hline $\begin{array}{l}\text { PredefinedType=; Name= } \\
\text { Pset_DistributionSystemCommon; }\end{array}$ & & & 0 & + & - & + & - & - & - & - & - & - & - & - & - & - & - & - & - & - & - & - & - \\
\hline $\begin{array}{l}\text { PredefinedType= } \\
\text { DOMESTICCOLDWATER; Name= } \\
\text { Pset_DistributionSystemTypePlumbing; }\end{array}$ & & & 0 & + & - & + & - & - & - & - & - & - & - & - & - & - & - & - & - & - & - & - & - \\
\hline Object Aggregation & $\mathrm{O}$ & & & & & & & & & & & & & & & & & & & & & & \\
\hline $\begin{array}{l}\text { PredefinedType=; } \\
\text { RelatedObjects=IfcDistributionCircuit; }\end{array}$ & & & 0 & + & - & + & - & - & - & - & - & - & - & - & - & - & - & - & - & - & - & - & - \\
\hline Group Assignment & $\mathrm{O}$ & & & & & & & & & & & & & & & & & & & & & & \\
\hline Type=IfcSanitaryTerminal; & & & 0 & + & - & + & - & - & - & - & - & - & - & - & - & - & - & - & - & - & - & - & - \\
\hline Type=IfcPump; & & & 0 & + & - & + & - & - & - & - & - & - & - & - & - & - & - & - & - & - & - & - & - \\
\hline Type=IfcValve; & & & 0 & + & - & + & - & - & - & - & - & - & - & - & - & - & - & - & - & - & - & - & - \\
\hline Type=IfcBoiler; & & & 0 & + & - & + & - & - & - & - & - & - & - & - & - & - & - & - & - & - & - & - & - \\
\hline Type=IfcWasteTerminal; & & & 0 & + & - & + & - & - & - & - & - & - & - & - & - & - & - & - & - & - & - & - & - \\
\hline Type=IfcFilter; & & & 0 & + & - & + & - & - & - & - & - & - & - & - & - & - & - & - & - & - & - & - & - \\
\hline IfcBoiler & & & 0 & & & & & & & & & & & & & & & & & & & & \\
\hline Identity & $\mathrm{R}$ & & 0 & + & - & - & - & + & + & + & + & + & + & + & + & + & + & + & + & + & + & + & + \\
\hline Object Typing & $\mathrm{R}$ & & & & & & & & & & & & & & & & & & & & & & \\
\hline
\end{tabular}




\begin{tabular}{|c|c|c|c|c|c|c|c|c|c|c|c|c|c|c|c|c|c|c|c|c|c|c|c|c|}
\hline Entity/Concept & Req & Errors & Count & Flag & 1 & 2 & 3 & 4 & 5 & 6 & 7 & 8 & 9 & $\begin{array}{l}1 \\
0\end{array}$ & $\begin{array}{l}1 \\
1\end{array}$ & $\begin{array}{l}1 \\
2\end{array}$ & $\begin{array}{l}1 \\
3\end{array}$ & $\begin{array}{l}1 \\
4\end{array}$ & $\begin{array}{l}1 \\
5\end{array}$ & 1 & & & $\begin{array}{l}1 \\
8\end{array}$ & $\begin{array}{l}1 \\
9\end{array}$ \\
\hline Type=IfcBoilerType; & & & 0 & + & - & - & - & + & + & + & + & + & + & + & + & + & + & + & + & + & & & + & + \\
\hline Property Sets & $\mathrm{R}$ & & & & & & & & & & & & & & & & & & & & & & & \\
\hline $\begin{array}{l}\text { PredefinedType=; } \\
\text { Name=Pset_BoilerPHistory; }\end{array}$ & & & 0 & + & - & - & - & - & - & + & + & + & + & + & + & + & + & + & + & + & & & + & + \\
\hline $\begin{array}{l}\text { Name=Pset_BoilerTypeCommon; } \\
\text { TemplateType= } \\
\text { PSET_TYPEDRIVENOVERRIDE; }\end{array}$ & & & 0 & + & - & - & - & - & - & + & + & + & + & + & + & + & + & + & + & + & & & + & + \\
\hline $\begin{array}{l}\text { PredefinedType=STEAM; } \\
\text { Name=Pset_BoilerTypeSteam; } \\
\text { TemplateType= } \\
\text { PSET_TYPEDRIVENOVERRIDE; }\end{array}$ & & & 0 & + & - & - & - & - & - & + & + & + & + & + & + & + & + & + & + & + & & & + & + \\
\hline $\begin{array}{l}\text { PredefinedType=WATER; } \\
\text { Name=Pset_BoilerTypeWater; }\end{array}$ & & & 0 & + & - & - & - & - & - & + & + & + & + & + & + & + & + & + & + & + & & & + & + \\
\hline Quantity Sets & $\mathrm{O}$ & & & & & & & & & & & & & & & & & & & & & & & \\
\hline Name=Qto_BoilerBaseQuantities; & & & 0 & + & - & - & - & - & - & - & - & - & - & - & - & - & - & - & - & - & & & - & + \\
\hline Ports [IFC4] & $\mathrm{R}$ & & & & & & & & & & & & & & & & & & & & & & & \\
\hline $\begin{array}{l}\text { PredefinedType=WATER; Name=Gas; } \\
\text { Flow=SINK; Type=GAS; }\end{array}$ & & & 0 & + & - & - & - & + & + & + & + & + & + & + & + & + & + & + & + & + & & & + & + \\
\hline $\begin{array}{l}\text { PredefinedType=WATER; Name= Ex- } \\
\text { haust; Type= EXHAUST; Flow=SOURCE }\end{array}$ & & & 0 & + & - & - & - & + & + & + & + & + & + & + & + & + & + & + & + & + & & & + & + \\
\hline $\begin{array}{l}\text { PredefinedType=WATER;Name }= \\
\text { ColdWater;Type= } \\
\text { DOMESTICCOLDWATER; Flow=SINK }\end{array}$ & & & 0 & + & - & - & - & + & + & + & + & + & + & + & + & + & + & + & + & + & & & + & + \\
\hline $\begin{array}{l}\text { PredefinedType=WATER;Name= } \\
\text { HotWater;Type= } \\
\text { DOMESTICHOTWATER;Flow=SOURCE }\end{array}$ & & & 0 & + & - & - & - & + & + & + & + & + & + & + & + & + & + & + & + & + & & & + & + \\
\hline Body Geometry & $\mathrm{R}$ & & & & & & & & & & & & & & & & & & & & & & & \\
\hline $\begin{array}{l}\text { RepresentationType= } \\
\text { MappedRepresentation; Geometry= } \\
\text { IfcMappedItem; }\end{array}$ & & & 0 & + & - & - & - & - & - & - & - & - & - & + & + & + & + & + & + & + & & & + & + \\
\hline Clearance Geometry & $\mathrm{O}$ & & 0 & + & - & - & - & - & - & - & - & - & - & + & + & + & + & + & + & + & & & + & + \\
\hline IfcBoilerType & & & 0 & & & & & & & & & & & & & & & & & & & & & \\
\hline Identity & $\mathrm{R}$ & & 0 & + & - & - & - & - & - & - & - & - & - & - & - & - & - & - & - & - & & & + & + \\
\hline IfcFlowMeter & & & 0 & & & & & & & & & & & & & & & & & & & & & \\
\hline Identity & $\mathrm{R}$ & & 0 & + & - & - & - & - & - & - & - & - & - & + & + & + & + & + & + & + & & & + & + \\
\hline Object Typing & $\mathrm{R}$ & & & & & & & & & & & & & & & & & & & & & & & \\
\hline
\end{tabular}




\begin{tabular}{|c|c|c|c|c|c|c|c|c|c|c|c|c|c|c|c|c|c|c|c|c|c|c|c|c|}
\hline Entity/Concept & Req & Errors & Count & Flag & 1 & 2 & 3 & 4 & 5 & 6 & 7 & 8 & 9 & $\begin{array}{l}1 \\
0\end{array}$ & $\begin{array}{l}1 \\
1\end{array}$ & $\begin{array}{l}1 \\
2\end{array}$ & $\begin{array}{l}1 \\
3\end{array}$ & $\begin{array}{l}1 \\
4\end{array}$ & $\begin{array}{l}1 \\
\mathbf{5}\end{array}$ & $\begin{array}{l}1 \\
6\end{array}$ & $\frac{1}{7}$ & & & $\begin{array}{l}1 \\
9\end{array}$ \\
\hline Type=IfcFlowMeterType; & & & 0 & + & - & - & - & - & - & - & - & - & - & - & - & - & - & - & - & - & - & & & + \\
\hline Property Sets & $\mathrm{O}$ & & & & & & & & & & & & & & & & & & & & & & & \\
\hline $\begin{array}{l}\text { PredefinedType=; } \\
\text { Name=Pset_FlowMeterOccurrence; }\end{array}$ & & & 0 & + & - & - & - & - & - & - & - & - & - & + & + & + & + & + & + & + & + & & & + \\
\hline $\begin{array}{l}\text { Name=Pset_FlowMeterTypeCommon; } \\
\text { TemplateType= } \\
\text { PSET_TYPEDRIVENOVERRIDE; }\end{array}$ & & & 0 & + & - & - & - & - & - & - & - & - & - & + & + & + & + & + & + & + & + & & & + \\
\hline $\begin{array}{l}\text { PredefinedType=ENERGYMETER; } \\
\text { Name= } \\
\text { Pset_FlowMeterTypeEnergyMeter; }\end{array}$ & & & 0 & + & - & - & - & - & - & - & - & - & - & + & + & + & + & + & + & + & + & & & + \\
\hline Ports [IFC4] & $\mathrm{R}$ & & & & & & & & & & & & & & & & & & & & & & & \\
\hline $\begin{array}{l}\text { PredefinedType=GASMETER; } \\
\text { Name=Inlet; Flow=SINK; Type=GAS; }\end{array}$ & & & 0 & + & - & - & - & - & - & - & - & - & - & + & + & + & + & + & + & + & + & & & + \\
\hline $\begin{array}{l}\text { PredefinedType=GASMETER;Name=Out } \\
\text { let; Type=GAS; Flow=SOURCE }\end{array}$ & & & 0 & + & - & - & - & - & - & - & - & - & - & + & + & + & + & + & + & + & + & & & + \\
\hline $\begin{array}{l}\text { PredefinedType=WATERMETER; } \\
\text { Name=Inlet; Flow=SINK; } \\
\text { Type=DOMESTICCOLDWATER; }\end{array}$ & & & 0 & + & - & - & - & - & - & - & - & - & - & + & + & + & + & + & + & + & + & & & + \\
\hline $\begin{array}{l}\text { PredefinedType=WATERMETER; } \\
\text { Name=Outlet;Type= } \\
\text { DOMESTICCOLDWATER; } \\
\text { Flow=SOURCE }\end{array}$ & & & 0 & + & - & - & - & - & - & - & - & - & - & + & + & + & + & + & + & + & + & & & + \\
\hline Product Placement & - & & & & & & & & & & & & & & & & & & & & & & & \\
\hline Type=IfcLocalPlacement; & & & 0 & - & - & - & - & - & - & - & - & - & - & - & - & - & - & - & - & - & - & & & - \\
\hline Body Geometry & $\mathrm{R}$ & & & & & & & & & & & & & & & & & & & & & & & \\
\hline $\begin{array}{l}\text { RepresentationType= } \\
\text { MappedRepresentation; Geometry= } \\
\text { IfcMappedltem; }\end{array}$ & & & 0 & + & - & - & - & - & - & - & - & - & - & + & + & + & + & + & + & + & + & & & + \\
\hline Spatial Containment & $\mathrm{R}$ & & & & & & & & & & & & & & & & & & & & & & & \\
\hline Structure=IfcSite; & & & 0 & + & - & - & - & - & - & - & - & - & - & + & + & + & + & + & + & + & + & & & + \\
\hline IfcFlowMeterType & & & 0 & & & & & & & & & & & & & & & & & & & & & \\
\hline Identity & - & & 0 & - & - & - & - & - & - & - & - & - & - & - & - & - & - & - & - & - & - & & & - \\
\hline Property Sets for Types & $\mathrm{R}$ & & 0 & + & - & - & - & - & - & - & - & - & - & - & - & - & - & - & - & - & - & & & + \\
\hline Type-Based Ports [IFC4] & $\mathrm{R}$ & & 0 & + & - & - & - & - & - & - & - & - & - & - & - & - & - & - & - & - & - & & & + \\
\hline IfcValve & & & 0 & & & & & & & & & & & & & & & & & & & & & \\
\hline Identity & $\mathrm{R}$ & & 0 & + & - & - & - & + & + & + & + & + & + & + & + & + & + & + & + & + & + & & & + \\
\hline
\end{tabular}




\begin{tabular}{|c|c|c|c|c|c|c|c|c|c|c|c|c|c|c|c|c|c|c|c|c|c|c|c|}
\hline Entity/Concept & Req & Errors & Count & Flag & 1 & 2 & 3 & 4 & 5 & 6 & 7 & 8 & 9 & $\begin{array}{l}1 \\
0\end{array}$ & $\begin{array}{l}1 \\
1\end{array}$ & 1 & & $\begin{array}{l}1 \\
4\end{array}$ & $\begin{array}{l}1 \\
5\end{array}$ & $\begin{array}{l}1 \\
6\end{array}$ & $\begin{array}{l}1 \\
7\end{array}$ & 1 & $\begin{array}{l}1 \\
9\end{array}$ \\
\hline Object Typing & $\mathrm{R}$ & & & & & & & & & & & & & & & & & & & & & & \\
\hline Type=IfcValveType; & & & 0 & + & - & - & - & - & - & - & - & - & - & - & - & - & 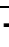 & - & - & - & - & + & + \\
\hline Property Sets & $\mathrm{R}$ & & & & & & & & & & & & & & & & & & & & & & \\
\hline $\begin{array}{l}\text { PredefinedType=; } \\
\text { Name=Pset_ValvePHistory; }\end{array}$ & & & 0 & + & - & - & - & + & + & + & + & + & + & + & + & 7 & & + & + & + & + & + & + \\
\hline $\begin{array}{l}\text { PredefinedType=AIRRELEASE; } \\
\text { Name=Pset_ValveTypeAirRelease; } \\
\text { TemplateType= } \\
\text { PSET_TYPEDRIVENOVERRIDE; }\end{array}$ & & & 0 & + & - & - & - & + & + & + & + & + & + & + & + & 7 & . & + & + & + & + & + & + \\
\hline $\begin{array}{l}\text { Name=Pset_ValveTypeCommon; } \\
\text { TemplateType= } \\
\text { PSET_TYPEDRIVENOVERRIDE; }\end{array}$ & & & 0 & + & - & - & - & + & + & + & + & + & + & + & + & 7 & . & + & + & + & + & + & + \\
\hline $\begin{array}{l}\text { PredefinedType=DRAWOFFCOCK; } \\
\text { Name=Pset_ValveTypeDrawOffCock; } \\
\text { TemplateType= } \\
\text { PSET_TYPEDRIVENOVERRIDE; }\end{array}$ & & & 0 & + & - & - & - & + & + & + & + & + & + & + & + & + & & + & + & + & + & + & + \\
\hline $\begin{array}{l}\text { PredefinedType=FAUCET; } \\
\text { Name=Pset_ValveTypeFaucet; } \\
\text { TemplateType= } \\
\text { PSET_TYPEDRIVENOVERRIDE; }\end{array}$ & & & 0 & + & - & - & - & + & + & + & + & + & + & + & + & + & & + & + & + & + & + & + \\
\hline $\begin{array}{l}\text { PredefinedType=FLUSHING; } \\
\text { Name=Pset_ValveTypeFlushing; } \\
\text { TemplateType= } \\
\text { PSET_TYPEDRIVENOVERRIDE; }\end{array}$ & & & 0 & + & - & - & - & + & + & + & + & + & + & + & + & + & & + & + & + & + & + & + \\
\hline $\begin{array}{l}\text { PredefinedType=GASTAP; } \\
\text { Name=Pset_ValveTypeGasTap; } \\
\text { TemplateType= } \\
\text { PSET_TYPEDRIVENOVERRIDE; }\end{array}$ & & & 0 & + & - & - & - & + & + & + & + & + & + & + & + & + & & + & + & + & + & + & + \\
\hline $\begin{array}{l}\text { PredefinedType=ISOLATING; } \\
\text { Name=Pset_ValveTypelsolating; } \\
\text { TemplateType=PSET_TYPEDRIVENOV } \\
\text { ERRIDE; }\end{array}$ & & & 0 & + & - & - & - & + & + & + & + & + & + & + & + & $t$ & & + & + & + & + & + & + \\
\hline $\begin{array}{l}\text { PredefinedType=MIXING; } \\
\text { Name=Pset_ValveTypeMixing; } \\
\text { TemplateType= } \\
\text { PSET_TYPEDRIVENOVERRIDE; }\end{array}$ & & & 0 & + & - & - & - & + & + & + & + & + & + & + & + & + & & + & + & + & + & + & + \\
\hline $\begin{array}{l}\text { PredefinedType= } \\
\text { PRESSUREREDUCING; Name= } \\
\text { Pset_ValveTypePressureReducing; } \\
\text { TemplateType= } \\
\text { PSET_TYPEDRIVENOVERRIDE; }\end{array}$ & & & 0 & + & - & - & - & + & + & + & + & + & + & + & + & 7 & & + & + & + & + & + & + \\
\hline $\begin{array}{l}\text { PredefinedType=PRESSURERELIEF; } \\
\text { Name=Pset_ValveTypePressureRelief; }\end{array}$ & & & 0 & + & - & - & - & + & + & + & + & + & + & + & + & +1 & & + & + & + & + & + & + \\
\hline
\end{tabular}




\begin{tabular}{|c|c|c|c|c|c|c|c|c|c|c|c|c|c|c|c|c|c|c|c|c|c|c|c|c|}
\hline Entity/Concept & Req & Errors & Count & Flag & 1 & 2 & 3 & 4 & 5 & 6 & 7 & 8 & 9 & $\begin{array}{l}1 \\
0\end{array}$ & $\begin{array}{l}1 \\
1\end{array}$ & $\begin{array}{l}1 \\
2\end{array}$ & $\begin{array}{l}1 \\
3\end{array}$ & 1 & & & $\begin{array}{l}1 \\
6\end{array}$ & $\begin{array}{l}1 \\
7\end{array}$ & $\begin{array}{l}1 \\
8\end{array}$ & $\begin{array}{l}1 \\
9\end{array}$ \\
\hline Quantity Sets & $\mathrm{O}$ & & & & & & & & & & & & & & & & & & & & & & & \\
\hline Name=Qto_ValveBaseQuantities; & & & 0 & + & - & - & - & - & - & - & - & - & - & - & - & - & - & - & & & 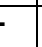 & - & - & + \\
\hline Material Constituents & - & & & & & & & & & & & & & & & & & & & & & & & \\
\hline Name=Casing; & & & 0 & - & - & - & - & - & - & - & - & - & - & - & - & - & - & - & & & 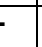 & - & - & - \\
\hline Name=Operation; & & & 0 & - & - & - & - & - & - & - & - & - & - & - & - & - & - & - & & & - & - & - & - \\
\hline Connectivity & $\mathrm{R}$ & & & & & & & & & & & & & & & & & & & & & & & \\
\hline $\begin{array}{l}\text { Type=IfcActuator; Relation= } \\
\text { IfcRelFlowControlElements; }\end{array}$ & & & 0 & + & - & - & - & - & - & - & - & - & - & - & - & + & + & + & & & + & + & + & + \\
\hline Ports [IFC4] & $\mathrm{R}$ & & & & & & & & & & & & & & & & & & & & & & & \\
\hline $\begin{array}{l}\text { PredefinedType=AIRHANDLER; } \\
\text { Name=Inlet; Type=NOTDEFINED; } \\
\text { Flow=SINK }\end{array}$ & & & 0 & + & - & - & - & + & + & + & + & + & + & + & + & + & + & + & & & + & + & + & + \\
\hline $\begin{array}{l}\text { PredefinedType=ANTIVACUUM; } \\
\text { Name==Outlet; Type=NOTDEFINED; } \\
\text { Flow=SOURCE }\end{array}$ & & & 0 & + & - & - & - & + & + & + & + & + & + & + & + & + & + & + & & & + & + & + & + \\
\hline $\begin{array}{l}\text { PredefinedType=CHANGEOVER; } \\
\text { Name=Inlet; Type=NOTDEFINED; } \\
\text { Flow=SINK }\end{array}$ & & & 0 & + & - & - & - & + & + & + & + & + & + & + & + & + & + & + & & & + & + & + & + \\
\hline $\begin{array}{l}\text { PredefinedType=CHANGEOVER; } \\
\text { Name=Outlet\#1; Type=NOTDEFINED; } \\
\text { Flow=SINK }\end{array}$ & & & 0 & + & - & - & - & + & + & + & + & + & + & + & + & + & + & + & & & + & + & + & + \\
\hline $\begin{array}{l}\text { PredefinedType=CHANGEOVER; } \\
\text { Name=Outlet\#2; Type=NOTDEFINED; } \\
\text { Flow=SINK }\end{array}$ & & & 0 & + & - & - & - & + & + & + & + & + & + & + & + & + & + & + & & & + & + & + & + \\
\hline $\begin{array}{l}\text { PredefinedType=CHECK; Name=Inlet; } \\
\text { Type=NOTDEFINED; Flow=SINK }\end{array}$ & & & 0 & + & - & - & - & + & + & + & + & + & + & + & + & + & + & + & & & + & + & + & + \\
\hline $\begin{array}{l}\text { PredefinedType=CHECK; Name=Outlet; } \\
\text { Type=NOTDEFINED; Flow=SINK }\end{array}$ & & & 0 & + & - & - & - & + & + & + & + & + & + & + & + & + & + & + & & & + & + & + & + \\
\hline $\begin{array}{l}\text { PredefinedType=COMMISSIONING; } \\
\text { Name=Inlet; Type=NOTDEFINED; } \\
\text { Flow=SINK }\end{array}$ & & & 0 & + & - & - & - & + & + & + & + & + & + & + & + & + & + & + & & & + & + & + & + \\
\hline $\begin{array}{l}\text { PredefinedType=COMMISSIONING; } \\
\text { Name=Outlet; Type=NOTDEFINED; } \\
\text { Flow=SOURCE }\end{array}$ & & & 0 & + & - & - & - & + & + & + & + & + & + & + & + & + & + & + & & & + & + & + & + \\
\hline $\begin{array}{l}\text { PredefinedType=DIVERTING; } \\
\text { Name=Inlet; Type=NOTDEFINED; } \\
\text { Flow=SINK }\end{array}$ & & & 0 & + & - & - & - & + & + & + & + & + & + & + & + & + & + & + & & & + & + & + & + \\
\hline $\begin{array}{l}\text { PredefinedType=DIVERTING; } \\
\text { Name=Outlet\#1; Type=NOTDEFINED; } \\
\text { Flow=SINK }\end{array}$ & & & 0 & + & - & - & - & + & + & + & + & + & + & + & + & + & + & + & & & + & + & + & + \\
\hline
\end{tabular}




\begin{tabular}{|c|c|c|c|c|c|c|c|c|c|c|c|c|c|c|c|c|c|c|c|c|c|c|c|c|}
\hline Entity/Concept & Req & Errors & Count & Flag & 1 & 2 & 3 & 4 & 5 & 6 & 7 & 8 & 9 & $\begin{array}{l}1 \\
0\end{array}$ & $\begin{array}{l}1 \\
1\end{array}$ & $\begin{array}{l}1 \\
2\end{array}$ & $\begin{array}{l}1 \\
3\end{array}$ & $\begin{array}{l}1 \\
4\end{array}$ & $\begin{array}{l}1 \\
5\end{array}$ & $\begin{array}{l}1 \\
6\end{array}$ & & & $\begin{array}{l}1 \\
8\end{array}$ & $\begin{array}{l}1 \\
9\end{array}$ \\
\hline $\begin{array}{l}\text { PredefinedType=DIVERTING; } \\
\text { Name=Outlet\#2; Type=NOTDEFINED; } \\
\text { Flow=SINK }\end{array}$ & & & 0 & + & - & - & - & + & + & + & + & + & + & + & + & + & + & + & + & + & & & + & + \\
\hline $\begin{array}{l}\text { PredefinedType=DOUBLECHECK; } \\
\text { Name=Inlet; Type=NOTDEFINED; } \\
\text { Flow=SINK }\end{array}$ & & & 0 & + & - & - & - & + & + & + & + & + & + & + & + & + & + & + & + & + & & & + & + \\
\hline $\begin{array}{l}\text { PredefinedType=DOUBLECHECK; } \\
\text { Name=Outlet; Type=NOTDEFINED; } \\
\text { Flow=SINK }\end{array}$ & & & 0 & + & - & - & - & + & + & + & + & + & + & + & + & + & + & + & + & + & & & + & + \\
\hline $\begin{array}{l}\text { PredefinedType= } \\
\text { DOUBLEREGULATING; Name=Inlet; } \\
\text { Type=NOTDEFINED; Flow=SINK }\end{array}$ & & & 0 & + & - & - & - & + & + & + & + & + & + & + & + & + & + & + & + & + & & & + & + \\
\hline $\begin{array}{l}\text { PredefinedType= } \\
\text { DOUBLEREGULATING; Name==Outlet; } \\
\text { Type=NOTDEFINED; Flow=SINK }\end{array}$ & & & 0 & + & - & - & - & + & + & + & + & + & + & + & + & + & + & + & + & + & & & + & + \\
\hline $\begin{array}{l}\text { PredefinedType=DRAWOFFCOCK; } \\
\text { Name=Inlet; Type=NOTDEFINED; } \\
\text { Flow=SINK }\end{array}$ & & & 0 & + & - & - & - & + & + & + & + & + & + & + & + & + & + & + & + & + & & & + & + \\
\hline $\begin{array}{l}\text { PredefinedType=FAUCET; Name=Inlet; } \\
\text { Type=NOTDEFINED; Flow=SINK }\end{array}$ & & & 0 & + & - & - & - & + & + & + & + & + & + & + & + & + & + & + & + & + & & & + & + \\
\hline $\begin{array}{l}\text { PredefinedType=FLUSHING; } \\
\text { Name=Inlet; Type=NOTDEFINED; } \\
\text { Flow=SINK }\end{array}$ & & & 0 & + & - & - & - & + & + & + & + & + & + & + & + & + & + & + & + & + & & & + & + \\
\hline $\begin{array}{l}\text { PredefinedType=FLUSHING; } \\
\text { Name=Outlet; Type=NOTDEFINED; } \\
\text { Flow=SINK }\end{array}$ & & & 0 & + & - & - & - & + & + & + & + & + & + & + & + & + & + & + & + & + & & & + & + \\
\hline $\begin{array}{l}\text { PredefinedType=GASCOCK; } \\
\text { Name=Inlet; Type=GAS; Flow=SINK }\end{array}$ & & & 0 & + & - & - & - & + & + & + & + & + & + & + & + & + & + & + & + & + & & & + & + \\
\hline $\begin{array}{l}\text { PredefinedType=GASTAP; Name=Inlet; } \\
\text { Type=GAS; Flow=SINK }\end{array}$ & & & 0 & + & - & - & - & + & + & + & + & + & + & + & + & + & + & + & + & + & & & + & + \\
\hline $\begin{array}{l}\text { PredefinedType=ISOLATING; } \\
\text { Name=Inlet; Type=GAS; Flow=SINK }\end{array}$ & & & 0 & + & - & - & - & + & + & + & + & + & + & + & + & + & + & + & + & + & & & + & + \\
\hline $\begin{array}{l}\text { PredefinedType=ISOLATING; } \\
\text { Name=Outlet; Type=GAS; } \\
\text { Flow=SOURCE }\end{array}$ & & & 0 & + & - & - & - & + & + & + & + & + & + & + & + & + & + & + & + & + & & & + & + \\
\hline $\begin{array}{l}\text { PredefinedType=MIXING; } \\
\text { Name=Inlet\#1; Type=NOTDEFINED; } \\
\text { Flow=SINK }\end{array}$ & & & 0 & + & - & - & - & + & + & + & + & + & + & + & + & + & + & + & + & + & & & + & + \\
\hline $\begin{array}{l}\text { PredefinedType=MIXING; } \\
\text { Name=Inlet\#2; Type=NOTDEFINED; } \\
\text { Flow=SINK }\end{array}$ & & & 0 & + & - & - & - & + & + & + & + & + & + & + & + & + & + & + & + & + & & & + & + \\
\hline $\begin{array}{l}\text { PredefinedType=MIXING; Name=Outlet; } \\
\text { Type=NOTDEFINED; Flow=SOURCE }\end{array}$ & & & 0 & + & - & - & - & + & + & + & + & + & + & + & + & + & + & + & + & + & & & + & + \\
\hline
\end{tabular}




\begin{tabular}{|c|c|c|c|c|c|c|c|c|c|c|c|c|c|c|c|c|c|c|c|c|c|c|c|}
\hline Entity/Concept & Req & Errors & Count & Flag & 1 & 2 & 3 & 4 & 5 & 6 & 7 & 8 & 9 & $\begin{array}{l}1 \\
0\end{array}$ & $\begin{array}{l}1 \\
1\end{array}$ & $\begin{array}{l}1 \\
2\end{array}$ & $\begin{array}{l}1 \\
3\end{array}$ & $\begin{array}{l}1 \\
4\end{array}$ & $\begin{array}{l}1 \\
5\end{array}$ & $\begin{array}{l}1 \\
6\end{array}$ & $\begin{array}{l}1 \\
7\end{array}$ & $\begin{array}{l}1 \\
8\end{array}$ & $\begin{array}{l}1 \\
9\end{array}$ \\
\hline $\begin{array}{l}\text { PredefinedType= } \\
\text { PRESSUREREDUCING; Name=Inlet; } \\
\text { Type=NOTDEFINED; Flow=SINK }\end{array}$ & & & 0 & + & - & - & - & + & + & + & + & + & + & + & + & + & + & + & + & + & + & + & + \\
\hline $\begin{array}{l}\text { PredefinedType= } \\
\text { PRESSUREREDUCING; Name=Outlet; } \\
\text { Type=NOTDEFINED; Flow=SOURCE }\end{array}$ & & & 0 & + & - & - & - & + & + & + & + & + & + & + & + & + & + & + & + & + & + & + & + \\
\hline $\begin{array}{l}\text { PredefinedType=PRESSURERELIEF; } \\
\text { Name=Inlet; Type=NOTDEFINED; } \\
\text { Flow=SINK }\end{array}$ & & & 0 & + & - & - & - & + & + & + & + & + & + & + & + & + & + & + & + & + & + & + & + \\
\hline $\begin{array}{l}\text { PredefinedType=REGULATING; } \\
\text { Name=Inlet; Type=NOTDEFINED; } \\
\text { Flow=SINK }\end{array}$ & & & 0 & + & - & - & - & + & + & + & + & + & + & + & + & + & + & + & + & + & + & + & + \\
\hline $\begin{array}{l}\text { PredefinedType=REGULATING; } \\
\text { Name=Inlet; Type=NOTDEFINED; } \\
\text { Flow=SOURCE }\end{array}$ & & & 0 & + & - & - & - & + & + & + & + & + & + & + & + & + & + & + & + & + & + & + & + \\
\hline $\begin{array}{l}\text { PredefinedType=SAFETYCUTOFF; } \\
\text { Name=Inlet; Type=NOTDEFINED; } \\
\text { Flow=SINK }\end{array}$ & & & 0 & + & - & - & - & + & + & + & + & + & + & + & + & + & + & + & + & + & + & + & + \\
\hline $\begin{array}{l}\text { PredefinedType=SAFETYCUTOFF; } \\
\text { Name=Outlet; Type=NOTDEFINED; } \\
\text { Flow=SOURCE }\end{array}$ & & & 0 & + & - & - & - & + & + & + & + & + & + & + & + & + & + & + & + & + & + & + & + \\
\hline $\begin{array}{l}\text { PredefinedType=STEAMTRAP; } \\
\text { Name=Inlet; Type=NOTDEFINED; } \\
\text { Flow=SINK }\end{array}$ & & & 0 & + & - & - & - & + & + & + & + & + & + & + & + & + & + & + & + & + & + & + & + \\
\hline $\begin{array}{l}\text { PredefinedType=STEAMTRAP; } \\
\text { Name=Outlet; Type=NOTDEFINED; } \\
\text { Flow=SOURCE }\end{array}$ & & & 0 & + & - & - & - & + & + & + & + & + & + & + & + & + & + & + & + & + & + & + & + \\
\hline $\begin{array}{l}\text { PredefinedType=STOPCOCK; } \\
\text { Name=Inlet; Type=NOTDEFINED; } \\
\text { Flow=SINK }\end{array}$ & & & 0 & + & - & - & - & + & + & + & + & + & + & + & + & + & + & + & + & + & + & + & + \\
\hline Body Geometry & $\mathrm{R}$ & & & & & & & & & & & & & & & & & & & & & & \\
\hline $\begin{array}{l}\text { RepresentationType= } \\
\text { MappedRepresentation; Geometry= } \\
\text { IfcMappedItem; }\end{array}$ & & & 0 & + & - & - & - & - & - & - & - & - & - & + & + & + & + & + & + & + & + & + & + \\
\hline IfcValveType & & & 10 & & & & & & & & & & & & & & & & & & & & \\
\hline Identity & $\mathrm{R}$ & & 10 & + & - & - & - & - & - & - & - & - & - & - & - & - & - & - & - & - & - & + & + \\
\hline Property Sets for Types & $\mathrm{R}$ & $\begin{array}{l}\# 6540 \\
54\end{array}$ & 0 & $\mathbf{F}$ & - & - & - & - & - & - & - & - & - & - & - & - & - & - & - & - & - & $\mathbf{F}$ & $\mathbf{F}$ \\
\hline Type-Based Ports [IFC4] & $\mathrm{R}$ & $\begin{array}{l}\# 6540 \\
54 \\
\end{array}$ & 0 & $F$ & - & - & - & - & - & - & - & - & - & - & - & - & - & - & - & - & - & $\mathbf{F}$ & $\mathbf{F}$ \\
\hline IfcWorkCalendar & & & 0 & & & & & & & & & & & & & & & & & & & & \\
\hline Identity & $\mathrm{R}$ & & 0 & + & + & - & - & - & - & - & - & - & - & - & - & - & - & - & - & - & - & - & - \\
\hline
\end{tabular}




\begin{tabular}{|c|c|c|c|c|c|c|c|c|c|c|c|c|c|c|c|c|c|c|c|c|c|c|c|}
\hline Entity/Concept & Req & Errors & Count & Flag & 1 & 2 & 3 & 4 & 5 & 6 & 7 & 8 & 9 & $\begin{array}{l}1 \\
0\end{array}$ & $\begin{array}{l}1 \\
1\end{array}$ & $\begin{array}{l}1 \\
2\end{array}$ & $\begin{array}{l}1 \\
3\end{array}$ & $\begin{array}{l}1 \\
4\end{array}$ & $\begin{array}{l}1 \\
5\end{array}$ & $\begin{array}{l}1 \\
6\end{array}$ & $\begin{array}{l}1 \\
7\end{array}$ & $\begin{array}{l}1 \\
8\end{array}$ & $\begin{array}{l}1 \\
9\end{array}$ \\
\hline Object Nesting & $\mathrm{O}$ & & & & & & & & & & & & & & & & & & & & & & \\
\hline Type=lfcCostSchedule; & & & 0 & + & + & - & - & - & - & - & - & - & - & - & - & - & - & - & - & - & - & - & - \\
\hline Calendar & $\mathrm{R}$ & & 0 & + & + & - & - & - & - & - & - & - & - & - & - & - & - & - & - & - & - & - & - \\
\hline Control Assignment & $\mathrm{R}$ & & & & & & & & & & & & & & & & & & & & & & \\
\hline Type=IfcSpatialStructureElement; & & & 0 & + & + & - & - & - & - & - & - & - & - & - & - & - & - & - & - & - & - & - & - \\
\hline IfcSubContractResourceType & & & 0 & & & & & & & & & & & & & & & & & & & & \\
\hline Identity & $\mathrm{R}$ & & 0 & + & - & + & - & - & - & - & - & - & - & - & - & - & - & - & - & - & - & - & - \\
\hline Resource Cost Rate & $\mathrm{R}$ & & 0 & + & - & + & - & - & - & - & - & - & - & - & - & - & - & - & - & - & - & - & - \\
\hline IfcSubContractResource & & & 0 & & & & & & & & & & & & & & & & & & & & \\
\hline Identity & $\mathrm{R}$ & & 0 & + & - & + & - & - & - & - & - & - & - & - & - & - & - & - & - & - & - & - & - \\
\hline Object Typing & - & & 0 & - & - & - & - & - & - & - & - & - & - & - & - & - & - & - & - & - & - & - & - \\
\hline Resource Cost & - & & 0 & - & - & - & - & - & - & - & - & - & - & - & - & - & - & - & - & - & - & - & - \\
\hline Resource Quantity & - & & 0 & - & - & - & - & - & - & - & - & - & - & - & - & - & - & - & - & - & - & - & - \\
\hline Resource Assignment & - & & & & & & & & & & & & & & & & & & & & & & \\
\hline Type=IfcActor; & & & 0 & - & - & - & - & - & - & - & - & - & - & - & - & - & - & - & - & - & - & - & - \\
\hline IfcBuildingStorey & & & 3 & & & & & & & & & & & & & & & & & & & & \\
\hline Identity & $\mathrm{R}$ & & 3 & + & + & + & + & + & + & + & + & + & + & + & + & + & + & + & + & + & + & + & + \\
\hline Property Sets & $\mathrm{O}$ & & & & & & & & & & & & & & & & & & & & & & \\
\hline $\begin{array}{l}\text { Name=Pset_BuildingStoreyCommon; } \\
\text { TemplateType= } \\
\text { PSET_OCCURRENCEDRIVEN; }\end{array}$ & & \#62 & 0 & * & - & - & * & - & - & - & - & - & - & - & - & - & - & - & - & - & - & - & - \\
\hline Building Storey Elevation & $\mathrm{R}$ & & 3 & + & + & + & + & + & + & + & + & + & + & + & + & + & + & + & + & + & + & + & + \\
\hline Spatial Decomposition & $\mathrm{R}$ & & & & & & & & & & & & & & & & & & & & & & \\
\hline Spatial Parts=IfcSpace; & & $\# 62$ & 0 & $\mathbf{F}$ & - & - & $\mathbf{F}$ & * & $\mathbf{F}$ & $\mathbf{F}$ & * & * & * & * & * & $\mathbf{F}$ & * & * & * & * & $*$ & * & $\mathbf{F}$ \\
\hline IfcSpace & & & 0 & & & & & & & & & & & & & & & & & & & & \\
\hline Identity & $\mathrm{R}$ & & 0 & + & - & - & + & + & + & + & + & + & + & + & + & + & + & + & + & + & + & + & + \\
\hline Object Typing & $\mathrm{O}$ & & & & & & & & & & & & & & & & & & & & & & \\
\hline Type=IfcSpace Type; & & & 0 & + & - & - & + & + & + & + & + & + & + & + & + & + & + & + & + & + & + & + & + \\
\hline Classification [IFC4] & $\mathrm{R}$ & & & & & & & & & & & & & & & & & & & & & & \\
\hline
\end{tabular}




\begin{tabular}{|c|c|c|c|c|c|c|c|c|c|c|c|c|c|c|c|c|c|c|c|c|c|c|c|}
\hline Entity/Concept & Req & Errors & Count & Flag & 1 & 2 & 3 & 4 & 5 & 6 & 7 & 8 & 9 & $\begin{array}{l}1 \\
0\end{array}$ & $\begin{array}{l}1 \\
1\end{array}$ & $\begin{array}{l}1 \\
2\end{array}$ & $\begin{array}{l}1 \\
3\end{array}$ & $\begin{array}{l}1 \\
4\end{array}$ & $\begin{array}{l}1 \\
5\end{array}$ & $\begin{array}{l}1 \\
6\end{array}$ & $\begin{array}{l}1 \\
7\end{array}$ & $\begin{array}{l}1 \\
8\end{array}$ & $\begin{array}{l}1 \\
9\end{array}$ \\
\hline $\begin{array}{l}\text { Source=CSI; Name=OmniClass; To- } \\
\text { kens= 13-00 } 00 \text { 00; }\end{array}$ & & & 0 & + & + & + & + & + & + & + & + & + & + & + & + & + & + & + & + & + & + & + & + \\
\hline Property Sets & $\mathrm{O}$ & & & & & & & & & & & & & & & & & & & & & & \\
\hline $\begin{array}{l}\text { PredefinedType=;Name= } \\
\text { Pset_SpaceOccupancyRequirements; }\end{array}$ & & & 0 & + & - & - & + & + & + & + & + & + & + & + & + & + & + & + & + & + & + & + & + \\
\hline $\begin{array}{l}\text { PredefinedType=; } \\
\text { Name=Pset_SpaceCommon; }\end{array}$ & & & 0 & + & - & - & + & + & + & + & + & + & + & + & + & + & + & + & + & + & + & + & + \\
\hline Quantity Sets & $\mathrm{O}$ & & & & & & & & & & & & & & & & & & & & & & \\
\hline $\begin{array}{l}\text { Name=Qto_SpaceBaseQuantities; } \\
\text { TemplateType= } \\
\text { QTO_OCCURRENCEDRIVEN; }\end{array}$ & & & 0 & + & - & - & - & - & - & - & - & - & - & - & - & + & + & + & + & + & + & + & + \\
\hline Space Boundaries & - & & 0 & - & - & - & - & - & - & - & - & - & - & - & - & - & - & - & - & - & - & - & - \\
\hline Product Placement & $\mathrm{R}$ & & & & & & & & & & & & & & & & & & & & & & \\
\hline Type=IfcLocalPlacement; & & & 0 & + & - & - & - & - & - & - & - & - & - & - & - & + & + & + & + & + & + & + & + \\
\hline Footprint Geometry & $\mathrm{R}$ & & & & & & & & & & & & & & & & & & & & & & \\
\hline $\begin{array}{l}\text { RepresentationType=GeometricCurveSet } \\
\text {; Geometry=IfcGeometricCurveSet; }\end{array}$ & & & 0 & + & - & - & - & - & - & - & - & - & - & - & - & + & + & + & + & + & + & + & + \\
\hline Body Geometry & $\mathrm{R}$ & & & & & & & & & & & & & & & & & & & & & & \\
\hline $\begin{array}{l}\text { RepresentationType=SweptSolid; Ge- } \\
\text { ometry=IfcExtrudedAreaSolid; }\end{array}$ & & & 0 & + & - & - & - & - & - & - & - & - & - & - & - & + & + & + & + & + & + & + & + \\
\hline $\begin{array}{l}\text { RepresentationType=Clipping; Geome- } \\
\text { try= IfcBooleanClippingResult; }\end{array}$ & & & 0 & + & - & - & - & - & - & - & - & - & - & - & - & + & + & + & + & + & + & + & + \\
\hline $\begin{array}{l}\text { RepresentationType=Brep; Geometry= } \\
\text { IfcFacetedBrep; }\end{array}$ & & & 0 & + & - & - & - & - & - & - & - & - & - & - & - & + & + & + & + & + & + & + & + \\
\hline IfcElectricAppliance & & & 0 & & & & & & & & & & & & & & & & & & & & \\
\hline Identity & $\mathrm{R}$ & & 0 & + & - & - & + & + & + & + & + & + & + & + & + & + & + & + & + & + & + & + & + \\
\hline Object Typing & $\mathrm{R}$ & & & & & & & & & & & & & & & & & & & & & & \\
\hline Type=IfcElectricApplianceType; & & & 0 & + & - & - & - & - & - & - & - & - & - & - & - & - & - & - & - & - & - & + & + \\
\hline Property Sets & $\mathrm{R}$ & & & & & & & & & & & & & & & & & & & & & & \\
\hline $\begin{array}{l}\text { PredefinedType=; } \\
\text { Name=Pset_ElectricAppliancePHistory; }\end{array}$ & & & 0 & + & - & - & - & + & + & + & + & + & + & + & + & + & + & + & + & + & + & + & + \\
\hline $\begin{array}{l}\text { Name= } \\
\text { Pset_ElectricApplianceTypeCommon; } \\
\text { TemplateType= } \\
\text { PSET_TYPEDRIVENOVERRIDE; }\end{array}$ & & & 0 & + & - & - & - & + & + & + & + & + & + & + & + & + & + & + & + & + & + & + & + \\
\hline
\end{tabular}




\begin{tabular}{|c|c|c|c|c|c|c|c|c|c|c|c|c|c|c|c|c|c|c|c|c|c|c|c|c|}
\hline Entity/Concept & Req & Errors & Count & Flag & 1 & 2 & 3 & 4 & 5 & 6 & 7 & 8 & 9 & $\begin{array}{l}1 \\
0\end{array}$ & $\begin{array}{l}1 \\
1\end{array}$ & $\begin{array}{l}1 \\
2\end{array}$ & $\begin{array}{l}1 \\
3\end{array}$ & $\begin{array}{l}1 \\
4\end{array}$ & $\begin{array}{l}1 \\
5\end{array}$ & & & 7 & $\begin{array}{l}1 \\
8\end{array}$ & $\begin{array}{l}1 \\
9\end{array}$ \\
\hline $\begin{array}{l}\text { PredefinedType=DISHWASHER; } \\
\text { Name= } \\
\text { Pset_ElectricApplianceTypeDishwasher; }\end{array}$ & & & 0 & + & - & - & - & + & + & + & + & + & + & + & + & + & + & + & + & & & + & + & + \\
\hline Ports [IFC4] & $\mathrm{R}$ & & & & & & & & & & & & & & & & & & & & & & & \\
\hline $\begin{array}{l}\text { PredefinedType=DISHWASHER; } \\
\text { Name=HotWater; Flow=SINK; } \\
\text { Type=DOMESTICHOTWATER; }\end{array}$ & & & 0 & + & - & - & + & + & + & + & + & + & + & + & + & + & + & + & + & & & + & + & + \\
\hline $\begin{array}{l}\text { PredefinedType=DISHWASHER; } \\
\text { Name=Drainage; Type=DRAINAGE; } \\
\text { Flow=SOURCE }\end{array}$ & & & 0 & + & - & - & + & + & + & + & + & + & + & + & + & + & + & + & + & & & + & + & + \\
\hline $\begin{array}{l}\text { PredefinedType=FRIDGE_FREEZER; } \\
\text { Name=ColdWater; Flow=SINK; } \\
\text { Type=DOMESTICCOLDWATER; }\end{array}$ & & & 0 & + & - & - & + & + & + & + & + & + & + & + & + & + & + & + & + & & & + & + & + \\
\hline $\begin{array}{l}\text { PredefinedType=WASHINGMACHINE; } \\
\text { Name=ColdWater; Flow=SINK; } \\
\text { Type=DOMESTICCOLDWATER; }\end{array}$ & & & 0 & + & - & - & + & + & + & + & + & + & + & + & + & + & + & + & + & & & + & + & + \\
\hline $\begin{array}{l}\text { PredefinedType=WASHINGMACHINE; } \\
\text { Name=HotWater;Type= } \\
\text { DOMESTICHOTWATER; Flow=SINK }\end{array}$ & & & 0 & + & - & - & + & + & + & + & + & + & + & + & + & + & + & + & + & & & + & + & + \\
\hline $\begin{array}{l}\text { PredefinedType=WASHINGMACHINE; } \\
\text { Name=Drainage; Type=DRAINAGE; } \\
\text { Flow=SOURCE }\end{array}$ & & & 0 & + & - & - & + & + & + & + & + & + & + & + & + & + & + & + & + & & & + & + & + \\
\hline Body Geometry & $\mathrm{R}$ & & & & & & & & & & & & & & & & & & & & & & & \\
\hline $\begin{array}{l}\text { RepresentationType= } \\
\text { MappedRepresentation; Geometry= } \\
\text { IfcMappedItem; }\end{array}$ & & & 0 & + & - & - & - & - & - & - & - & - & - & + & + & + & + & + & + & & & + & + & + \\
\hline IfcElectricApplianceType & & & 0 & & & & & & & & & & & & & & & & & & & & & \\
\hline Identity & $\mathrm{R}$ & & 0 & + & - & - & - & - & - & - & - & - & - & - & - & - & - & - & - & & & - & + & + \\
\hline Property Sets for Types & $\mathrm{R}$ & & 0 & + & - & - & - & - & - & - & - & - & - & - & - & - & - & - & - & & & - & + & + \\
\hline Type-Based Ports [IFC4] & $\mathrm{R}$ & & 0 & + & - & - & - & - & - & - & - & - & - & - & - & - & - & - & - & & & 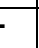 & + & + \\
\hline IfcSolarDevice & & & 0 & & & & & & & & & & & & & & & & & & & & & \\
\hline Identity & $\mathrm{R}$ & & 0 & + & - & - & - & - & - & - & - & - & - & + & + & + & + & + & + & & & + & + & + \\
\hline Object Typing & $\mathrm{R}$ & & & & & & & & & & & & & & & & & & & & & & & \\
\hline Type=IfcSolarDeviceType; & & & 0 & + & - & - & - & - & - & - & - & - & - & - & - & - & - & - & - & & & . & + & + \\
\hline Property Sets & $\mathrm{O}$ & & & & & & & & & & & & & & & & & & & & & & & \\
\hline $\begin{array}{l}\text { PredefinedType=; } \\
\text { Name=Pset_SolarDeviceTypeCommon; }\end{array}$ & & & 0 & + & - & - & - & - & - & - & - & - & - & + & + & + & + & + & + & & & + & + & + \\
\hline Ports [IFC4] & $\mathrm{R}$ & & & & & & & & & & & & & & & & & & & & & & & \\
\hline
\end{tabular}




\begin{tabular}{|c|c|c|c|c|c|c|c|c|c|c|c|c|c|c|c|c|c|c|c|c|c|c|c|}
\hline Entity/Concept & Req & Errors & Count & Flag & 1 & 2 & 3 & 4 & 5 & 6 & 7 & 8 & 9 & $\begin{array}{l}1 \\
0\end{array}$ & $\begin{array}{l}1 \\
1\end{array}$ & $\begin{array}{l}1 \\
2\end{array}$ & $\begin{array}{l}1 \\
3\end{array}$ & $\begin{array}{l}1 \\
4\end{array}$ & $\begin{array}{l}1 \\
5\end{array}$ & $\begin{array}{l}1 \\
6\end{array}$ & $\begin{array}{l}1 \\
7\end{array}$ & $\begin{array}{l}1 \\
8\end{array}$ & $\begin{array}{l}1 \\
9\end{array}$ \\
\hline $\begin{array}{l}\text { PredefinedType= SOLARCOLLECTOR; } \\
\text { Name=Inlet; Flow=SOURCE; } \\
\text { Type=HEATING; }\end{array}$ & & & 0 & + & - & - & - & - & - & - & - & - & - & + & + & + & + & + & + & + & + & + & + \\
\hline $\begin{array}{l}\text { PredefinedType= SOLARCOLLECTOR; } \\
\text { Name=Outlet; Flow=SINK; } \\
\text { Type=HEATING; }\end{array}$ & & & 0 & + & - & - & - & - & - & - & - & - & - & + & + & + & + & + & + & + & + & + & + \\
\hline Body Geometry & $\mathrm{R}$ & & & & & & & & & & & & & & & & & & & & & & \\
\hline $\begin{array}{l}\text { RepresentationType= } \\
\text { MappedRepresentation; Geometry= } \\
\text { IfcMappedltem; }\end{array}$ & & & 0 & + & - & - & - & - & - & - & - & - & - & + & + & + & + & + & + & + & + & + & + \\
\hline IfcFilterType & & & 0 & & & & & & & & & & & & & & & & & & & & \\
\hline Identity & $\mathrm{R}$ & & 0 & + & - & - & - & - & - & - & - & - & - & - & - & - & - & - & - & - & - & + & + \\
\hline Property Sets for Types & $\mathrm{R}$ & & 0 & + & - & - & - & - & - & - & - & - & - & - & - & - & - & - & - & - & - & + & + \\
\hline Type-Based Ports [IFC4] & $\mathrm{R}$ & & 0 & + & - & - & - & - & - & - & - & - & - & - & - & - & - & - & - & - & - & + & + \\
\hline IfcFilter & & & 0 & & & & & & & & & & & & & & & & & & & & \\
\hline Identity & $\mathrm{R}$ & & 0 & + & - & - & - & + & + & + & + & + & + & + & + & + & + & + & + & + & + & + & + \\
\hline Object Typing & $\mathrm{R}$ & & & & & & & & & & & & & & & & & & & & & & \\
\hline Type=IfcFilterType; & & & 0 & + & - & - & - & - & - & - & - & - & - & - & - & - & - & - & - & - & - & + & + \\
\hline Property Sets & $\mathrm{R}$ & & & & & & & & & & & & & & & & & & & & & & \\
\hline $\begin{array}{l}\text { PredefinedType=; } \\
\text { Name=Pset_FilterPHistory; }\end{array}$ & & & 0 & + & - & - & + & + & + & + & + & + & + & + & + & + & + & + & + & + & + & + & + \\
\hline $\begin{array}{l}\text { PredefinedType=; } \\
\text { Name=Pset_FilterTypeCommon; }\end{array}$ & & & 0 & + & - & - & + & + & + & + & + & + & + & + & + & + & + & + & + & + & + & + & + \\
\hline $\begin{array}{l}\text { PredefinedType=WATERFILTER; } \\
\text { Name=Pset_FilterTypeWaterFilter; }\end{array}$ & & & 0 & + & - & - & + & + & + & + & + & + & + & + & + & + & + & + & + & + & + & + & + \\
\hline Ports [IFC4] & $\mathrm{R}$ & & & & & & & & & & & & & & & & & & & & & & \\
\hline $\begin{array}{l}\text { PredefinedType=ODORFILTER;Name= } \\
\text { Inlet; Flow=SINK; Type=EXHAUST; }\end{array}$ & & & 0 & + & - & - & + & + & + & + & + & + & + & + & + & + & + & + & + & + & + & + & + \\
\hline $\begin{array}{l}\text { PredefinedType=ODORFILTER; } \\
\text { Name==Outlet; Type=EXHAUST; } \\
\text { Flow=SOURCE }\end{array}$ & & & 0 & + & - & - & + & + & + & + & + & + & + & + & + & + & + & + & + & + & + & + & + \\
\hline $\begin{array}{l}\text { PredefinedType=STRAINER; } \\
\text { Name=Inlet; Flow=SINK; } \\
\text { Type=DRAINAGE; }\end{array}$ & & & 0 & + & - & - & + & + & + & + & + & + & + & + & + & + & + & + & + & + & + & + & + \\
\hline $\begin{array}{l}\text { PredefinedType=STRAINER; } \\
\text { Name=Outlet; Type=DRAINAGE; } \\
\text { Flow=SOURCE }\end{array}$ & & & 0 & + & - & - & + & + & + & + & + & + & + & + & + & + & + & + & + & + & + & + & + \\
\hline
\end{tabular}




\begin{tabular}{|c|c|c|c|c|c|c|c|c|c|c|c|c|c|c|c|c|c|c|c|c|c|c|c|c|}
\hline Entity/Concept & Req & Errors & Count & Flag & 1 & 2 & 3 & 4 & 5 & 6 & 7 & 8 & 9 & $\begin{array}{l}1 \\
0\end{array}$ & $\begin{array}{l}1 \\
1\end{array}$ & $\begin{array}{l}1 \\
2\end{array}$ & $\begin{array}{l}1 \\
3\end{array}$ & $\begin{array}{l}1 \\
4\end{array}$ & $\begin{array}{l}1 \\
5\end{array}$ & $\begin{array}{l}1 \\
6\end{array}$ & & & $\begin{array}{l}1 \\
8\end{array}$ & $\begin{array}{l}1 \\
9\end{array}$ \\
\hline $\begin{array}{l}\text { PredefinedType=WATERFILTER; } \\
\text { Name=Inlet;Type= } \\
\text { DOMESTICCOLDWATER; Flow=SINK }\end{array}$ & & & 0 & + & - & - & + & + & + & + & + & + & + & + & + & + & + & + & + & + & & & + & + \\
\hline $\begin{array}{l}\text { PredefinedType=WATERFILTER; } \\
\text { Name=Outlet;Type= } \\
\text { DOMESTICCOLDWATER; } \\
\text { Flow=SOURCE }\end{array}$ & & & 0 & + & - & - & + & + & + & + & + & + & + & + & + & + & + & + & + & + & & & + & + \\
\hline Spatial Containment & $\mathrm{R}$ & & 0 & + & - & - & - & - & - & + & + & + & + & + & + & + & + & + & + & + & & & + & + \\
\hline Body Geometry & $\mathrm{R}$ & & & & & & & & & & & & & & & & & & & & & & & \\
\hline $\begin{array}{l}\text { RepresentationType= } \\
\text { MappedRepresentation; Geometry= } \\
\text { IfcMappedltem; }\end{array}$ & & & 0 & + & - & - & - & - & - & - & - & - & - & + & + & + & + & + & + & + & & & + & + \\
\hline IfcWasteTerminalType & & & 0 & & & & & & & & & & & & & & & & & & & & & \\
\hline Identity & $\mathrm{R}$ & & 0 & + & - & - & - & - & - & - & - & - & - & - & - & - & - & - & - & - & & & + & + \\
\hline Type-Based Ports [IFC4] & $\mathrm{R}$ & & 0 & + & - & - & - & - & - & - & - & - & - & - & - & - & - & - & - & - & & & + & + \\
\hline Body Geometry & $\mathrm{R}$ & & 0 & + & - & - & - & - & - & - & - & - & - & - & - & - & - & - & - & - & & & + & + \\
\hline Clearance Geometry & $\mathrm{R}$ & & & & & & & & & & & & & & & & & & & & & & & \\
\hline $\begin{array}{l}\text { Type=SurfaceModel; Geometry= } \\
\text { IfcFaceBasedSurfaceModel; }\end{array}$ & & & 0 & + & - & - & - & - & - & - & - & - & - & - & - & - & - & - & - & - & & & + & + \\
\hline IfcPerformanceHistory & & & 0 & & & & & & & & & & & & & & & & & & & & & \\
\hline Identity & $\mathrm{R}$ & & 0 & + & - & - & - & - & - & - & + & - & - & - & - & - & - & - & - & - & & & - & - \\
\hline Property Sets for Performance & $\mathrm{R}$ & & & & & & & & & & & & & & & & & & & & & & & \\
\hline $\begin{array}{l}\text { Name= } \\
\text { Pset_DistributionPortPHistoryPipe; }\end{array}$ & & & 0 & + & - & - & - & - & - & - & + & - & - & - & - & - & - & - & - & - & & & - & - \\
\hline $\begin{array}{l}\text { Name }= \\
\text { Pset_UtilityConsumptionPHistory; }\end{array}$ & & & 0 & + & - & - & - & - & - & - & + & - & - & - & - & - & - & - & - & - & & & - & - \\
\hline Control Assignment & $\mathrm{R}$ & & & & & & & & & & & & & & & & & & & & & & & \\
\hline Type=IfcGroup; & & & 0 & + & - & - & - & - & - & - & + & - & - & - & - & - & - & - & - & - & & & - & - \\
\hline Type=IfcProduct; & & & 0 & + & - & - & - & - & - & - & + & - & - & - & - & - & - & - & - & - & & & - & - \\
\hline IfcSystemFurnitureElement & & & 0 & & & & & & & & & & & & & & & & & & & & & \\
\hline Identity & $\mathrm{R}$ & & 0 & + & - & - & - & - & - & - & - & - & - & - & - & - & - & - & - & - & & & + & + \\
\hline Object Typing & $\mathrm{R}$ & & & & & & & & & & & & & & & & & & & & & & & \\
\hline Type= IfcSystemFurnitureElementType; & & & 0 & + & - & - & - & - & - & - & - & - & - & - & - & - & - & - & - & - & & & + & + \\
\hline Property Sets & $\mathrm{O}$ & & & & & & & & & & & & & & & & & & & & & & & \\
\hline
\end{tabular}




\begin{tabular}{|c|c|c|c|c|c|c|c|c|c|c|c|c|c|c|c|c|c|c|c|c|c|c|c|}
\hline Entity/Concept & Req & Errors & Count & Flag & 1 & 2 & 3 & 4 & 5 & 6 & 7 & 8 & 9 & $\begin{array}{l}1 \\
0\end{array}$ & $\begin{array}{l}1 \\
1\end{array}$ & $\begin{array}{l}1 \\
2\end{array}$ & $\begin{array}{l}1 \\
3\end{array}$ & $\begin{array}{l}1 \\
4\end{array}$ & $\begin{array}{l}1 \\
5\end{array}$ & $\begin{array}{l}1 \\
6\end{array}$ & $\begin{array}{l}1 \\
7\end{array}$ & $\begin{array}{l}\mathbf{1} \\
\mathbf{8}\end{array}$ & $\begin{array}{l}1 \\
9\end{array}$ \\
\hline $\begin{array}{l}\text { PredefinedType=; } \\
\text { Name=Pset_SystemFurniture } \\
\text { ElementTypeCommon; }\end{array}$ & & & 0 & + & - & - & - & - & - & - & - & - & - & - & - & - & - & - & - & - & - & + & + \\
\hline $\begin{array}{l}\text { PredefinedType=PANEL; } \\
\text { Name=Pset_SystemFurniture } \\
\text { ElementTypePanel; TemplateType= } \\
\text { PSET_TYPEDRIVENOVERRIDE; }\end{array}$ & & & 0 & + & - & - & - & - & - & - & - & - & - & - & - & - & - & - & - & - & - & + & + \\
\hline $\begin{array}{l}\text { PredefinedType=WORKSURFACE; } \\
\text { Name=Pset_SystemFurniture } \\
\text { ElementTypeWorkSurface; }\end{array}$ & & & 0 & + & - & - & - & - & - & - & - & - & - & - & - & - & - & - & - & - & - & + & + \\
\hline Material Constituents & $\mathrm{O}$ & & & & & & & & & & & & & & & & & & & & & & \\
\hline Name=Finish; & & & 0 & + & - & - & - & - & - & - & - & - & - & - & - & - & - & - & - & - & - & + & + \\
\hline Name=Frame; & & & 0 & + & - & - & - & - & - & - & - & - & - & - & - & - & - & - & - & - & - & + & + \\
\hline Name=Hardware; & & & 0 & + & - & - & - & - & - & - & - & - & - & - & - & - & - & - & - & - & - & + & + \\
\hline Name=Padding; & & & 0 & + & - & - & - & - & - & - & - & - & - & - & - & - & - & - & - & - & - & + & + \\
\hline Name=Panel; & & & 0 & + & - & - & - & - & - & - & - & - & - & - & - & - & - & - & - & - & - & + & + \\
\hline Product Placement & $\mathrm{R}$ & & 0 & + & - & - & - & - & - & - & - & - & - & - & - & - & - & - & - & - & - & + & + \\
\hline Body Geometry & $\mathrm{R}$ & & 0 & + & - & - & - & - & - & - & - & - & - & - & - & - & - & - & - & - & - & + & + \\
\hline IfcSystemFurnitureElementType & & & 0 & & & & & & & & & & & & & & & & & & & & \\
\hline Identity & $\mathrm{R}$ & & 0 & + & - & - & - & - & - & - & - & - & - & - & - & - & - & - & - & - & - & + & + \\
\hline IfcCostSchedule & & & 0 & & & & & & & & & & & & & & & & & & & & \\
\hline Identity & $\mathrm{R}$ & & 0 & + & - & + & - & - & - & - & - & - & - & - & - & - & - & - & - & - & - & - & - \\
\hline Object Nesting & $\mathrm{O}$ & & & & & & & & & & & & & & & & & & & & & & \\
\hline Type=IfcCostltem; & & & 0 & + & - & + & - & - & - & - & - & - & - & - & - & - & - & - & - & - & - & - & - \\
\hline IfcSolarDeviceType & & & 0 & & & & & & & & & & & & & & & & & & & & \\
\hline Identity & $\mathrm{R}$ & & 0 & + & - & - & - & - & - & - & - & - & - & - & - & - & - & - & - & - & - & + & + \\
\hline Property Sets for Types & $\mathrm{R}$ & & 0 & + & - & - & - & - & - & - & - & - & - & - & - & - & - & - & - & - & - & + & + \\
\hline Type-Based Ports [IFC4] & $\mathrm{R}$ & & 0 & + & - & - & - & - & - & - & - & - & - & - & - & - & - & - & - & - & - & + & + \\
\hline Body Geometry & $\mathrm{R}$ & & & & & & & & & & & & & & & & & & & & & & \\
\hline $\begin{array}{l}\text { RepresentationType=Brep; Geometry= } \\
\text { IfcFacetedBrep; }\end{array}$ & & & 0 & + & - & - & - & - & - & - & - & - & - & - & - & - & - & - & - & - & - & + & + \\
\hline Clearance Geometry & $\mathrm{O}$ & & & & & & & & & & & & & & & & & & & & & & \\
\hline
\end{tabular}




\begin{tabular}{|c|c|c|c|c|c|c|c|c|c|c|c|c|c|c|c|c|c|c|c|c|c|c|c|}
\hline Entity/Concept & Req & Errors & Count & Flag & 1 & 2 & 3 & 4 & 5 & 6 & 7 & 8 & 9 & $\begin{array}{l}1 \\
0\end{array}$ & $\begin{array}{l}1 \\
1\end{array}$ & $\begin{array}{l}1 \\
2\end{array}$ & $\begin{array}{l}1 \\
3\end{array}$ & $\begin{array}{l}1 \\
4\end{array}$ & $\begin{array}{l}1 \\
5\end{array}$ & $\begin{array}{l}1 \\
6\end{array}$ & $\begin{array}{l}1 \\
7\end{array}$ & $\begin{array}{l}1 \\
8\end{array}$ & $\begin{array}{l}1 \\
9\end{array}$ \\
\hline $\begin{array}{l}\text { Type=SurfaceModel; Geometry= } \\
\text { IfcFaceBasedSurfaceModel; }\end{array}$ & & & 0 & + & - & - & - & - & - & - & - & - & - & - & - & - & - & - & - & - & - & + & + \\
\hline IfcWallElementedCase & & & 0 & & & & & & & & & & & & & & & & & & & & \\
\hline Identity & $\mathrm{R}$ & & 0 & + & - & - & - & - & - & - & - & - & - & + & + & + & + & + & + & + & + & + & + \\
\hline Object Aggregation & $\mathrm{O}$ & & & & & & & & & & & & & & & & & & & & & & \\
\hline $\begin{array}{l}\text { PredefinedType=; } \\
\text { RelatedObjects=IfcMember; }\end{array}$ & & & 0 & + & - & - & - & - & - & - & - & - & - & + & + & + & + & + & + & + & + & + & + \\
\hline $\begin{array}{l}\text { PredefinedType=; } \\
\text { RelatedObjects=IfcPlate; }\end{array}$ & & & 0 & + & - & - & - & - & - & - & - & - & - & + & + & + & + & + & + & + & + & + & 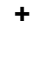 \\
\hline $\begin{array}{l}\text { PredefinedType=; } \\
\text { RelatedObjects=IfcBuildingElementPart; }\end{array}$ & & & 0 & + & - & - & - & - & - & - & - & - & - & + & + & + & + & + & + & + & + & + & + \\
\hline $\begin{array}{l}\text { PredefinedType=; RelatedObjects= } \\
\text { IfcBeam; }\end{array}$ & & & 0 & + & - & - & - & - & - & - & - & - & - & + & + & + & + & + & + & + & + & + & + \\
\hline Product Placement & $\mathrm{R}$ & & 0 & + & - & - & - & - & - & - & - & - & - & + & + & + & + & + & + & + & + & + & + \\
\hline Axis Geometry & $\mathrm{R}$ & & & & & & & & & & & & & & & & & & & & & & \\
\hline $\begin{array}{l}\text { RepresentationType=Curve2D; Geome- } \\
\text { try=IfcBoundedCurve; }\end{array}$ & & & 0 & + & - & - & - & - & - & - & - & - & - & + & + & + & + & + & + & + & + & + & + \\
\hline Surface Geometry & $\mathrm{O}$ & & & & & & & & & & & & & & & & & & & & & & \\
\hline $\begin{array}{l}\text { RepresentationType=Surface3D; Geom- } \\
\text { etry=IfcBoundedSurface; }\end{array}$ & & & 0 & + & - & - & - & - & - & - & - & - & - & + & + & + & + & + & + & + & + & 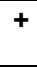 & + \\
\hline Spatial Containment & $\mathrm{R}$ & & & & & & & & & & & & & & & & & & & & & & \\
\hline Structure=IfcSite; & & & 0 & + & - & - & - & - & - & - & - & - & - & + & + & + & + & + & + & + & + & + & + \\
\hline Structure=IfcBuildingStorey; & & & 0 & + & - & - & - & - & - & - & - & - & - & + & + & + & + & + & + & + & + & + & + \\
\hline IfcSlabElementedCase & & & 0 & & & & & & & & & & & & & & & & & & & & \\
\hline Identity & $\mathrm{R}$ & & 0 & + & - & - & - & - & - & - & - & - & - & + & + & + & + & + & + & + & + & + & + \\
\hline Object Aggregation & $\mathrm{O}$ & & & & & & & & & & & & & & & & & & & & & & \\
\hline $\begin{array}{l}\text { PredefinedType=; } \\
\text { RelatedObjects=IfcBeam; }\end{array}$ & & & 0 & + & - & - & - & - & - & - & - & - & - & + & + & + & + & + & + & + & + & + & + \\
\hline $\begin{array}{l}\text { PredefinedType=; } \\
\text { RelatedObjects=IfcPlate; }\end{array}$ & & & 0 & + & - & - & - & - & - & - & - & - & - & + & + & + & + & + & + & + & + & + & + \\
\hline $\begin{array}{l}\text { PredefinedType=; } \\
\text { RelatedObjects=IfcBuildingElementPart; }\end{array}$ & & & 0 & + & - & - & - & - & - & - & - & - & - & + & + & + & + & + & + & + & + & + & + \\
\hline Product Placement & $\mathrm{R}$ & & 0 & + & - & - & - & - & - & - & - & - & - & + & + & + & + & + & + & + & + & + & + \\
\hline Surface Geometry & $\mathrm{O}$ & & & & & & & & & & & & & & & & & & & & & & \\
\hline
\end{tabular}




\begin{tabular}{|c|c|c|c|c|c|c|c|c|c|c|c|c|c|c|c|c|c|c|c|c|c|c|c|c|}
\hline Entity/Concept & Req & Errors & Count & Flag & 1 & 2 & 3 & 4 & 5 & 6 & 7 & 8 & 9 & $\begin{array}{l}1 \\
0\end{array}$ & $\begin{array}{l}1 \\
1\end{array}$ & $\begin{array}{l}1 \\
2\end{array}$ & $\begin{array}{l}1 \\
3\end{array}$ & $\begin{array}{l}1 \\
4\end{array}$ & $\begin{array}{l}1 \\
5\end{array}$ & $\begin{array}{l}1 \\
6\end{array}$ & $\frac{1}{7}$ & & & $\begin{array}{l}1 \\
9\end{array}$ \\
\hline $\begin{array}{l}\text { RepresentationType=Surface3D; Geom- } \\
\text { etry=IfcBoundedSurface; }\end{array}$ & & & 0 & + & - & - & - & - & - & - & - & - & - & + & + & + & + & + & + & + & + & & & + \\
\hline Spatial Containment & $\mathrm{R}$ & & & & & & & & & & & & & & & & & & & & & & & \\
\hline Structure=IfcBuildingStorey; & & & 0 & + & - & - & - & - & - & - & - & - & - & + & + & + & + & + & + & + & + & & & + \\
\hline IfcMember & & & 0 & & & & & & & & & & & & & & & & & & & & & \\
\hline Identity & $\mathrm{R}$ & & 0 & + & - & - & - & - & - & - & - & - & - & + & + & + & + & + & + & + & + & & & + \\
\hline Object Typing & $\mathrm{R}$ & & & & & & & & & & & & & & & & & & & & & & & \\
\hline Type=IfcMemberType; & & & 0 & + & - & - & - & - & - & - & - & - & - & - & - & - & - & - & - & - & - & & & + \\
\hline Property Sets & $\mathrm{O}$ & & & & & & & & & & & & & & & & & & & & & & & \\
\hline $\begin{array}{l}\text { Name=Pset_MemberCommon; } \\
\text { TemplateType= } \\
\text { PSET_TYPEDRIVENOVERRIDE; }\end{array}$ & & & 0 & + & - & - & - & - & - & - & - & - & - & - & - & - & - & - & - & - & - & & & + \\
\hline Material Profile Set [IFC4] & $\mathrm{R}$ & & 0 & + & - & - & - & - & - & - & - & - & - & - & - & - & - & - & - & + & + & & & + \\
\hline Product Placement & $\mathrm{R}$ & & 0 & + & - & - & - & - & - & - & - & - & - & - & - & - & - & - & - & + & + & & & + \\
\hline Axis Geometry & $\mathrm{R}$ & & & & & & & & & & & & & & & & & & & & & & & \\
\hline $\begin{array}{l}\text { Rep Identifier=Axis; Identifier=; } \\
\text { RepresentationType=Curve3D; Type=; } \\
\text { Geometry=IfcBoundedCurve; }\end{array}$ & & & 0 & + & - & - & - & - & - & - & - & - & - & - & - & - & - & - & - & + & + & & & + \\
\hline Body Geometry & $\mathrm{R}$ & & & & & & & & & & & & & & & & & & & & & & & \\
\hline $\begin{array}{l}\text { RepresentationType=SweptSolid; Ge- } \\
\text { ometry=IfcExtrudedAreaSolid; }\end{array}$ & & & 0 & + & - & - & - & - & - & - & - & - & - & - & - & - & - & - & - & + & + & & & + \\
\hline $\begin{array}{l}\text { RepresentationType=Clipping; Geome- } \\
\text { try=IfcBooleanClippingResult; }\end{array}$ & & & 0 & + & - & - & - & - & - & - & - & - & - & - & - & - & - & - & - & + & + & & & + \\
\hline $\begin{array}{l}\text { RepresentationType=AdvancedSweptSol } \\
\text { id; Geometry=IfcSweptAreaSolid; }\end{array}$ & & & 0 & + & - & - & - & - & - & - & - & - & - & - & - & - & - & - & - & + & + & & & + \\
\hline $\begin{array}{l}\text { RepresentationType=SurfaceModel; } \\
\text { Geometry=IfcFaceBasedSurfaceModel; }\end{array}$ & & & 0 & + & - & - & - & - & - & - & - & - & - & - & - & - & - & - & - & + & + & & & + \\
\hline $\begin{array}{l}\text { RepresentationType=Brep; Geome- } \\
\text { try=IfcFacetedBrep; }\end{array}$ & & & 0 & + & - & - & - & - & - & - & - & - & - & - & - & - & - & - & - & + & + & & & + \\
\hline $\begin{array}{l}\text { RepresentationType= } \\
\text { MappedRepresentation; Geometry= } \\
\text { IfcMappedItem; }\end{array}$ & & & 0 & + & - & - & - & - & - & - & - & - & - & - & - & - & - & - & - & + & + & & & + \\
\hline IfcSlabStandardCase & & & 0 & & & & & & & & & & & & & & & & & & & & & \\
\hline Identity & $\mathrm{R}$ & & 0 & + & - & - & - & - & - & - & - & - & - & + & + & + & + & + & + & + & + & & & + \\
\hline Object Typing & $\mathrm{O}$ & & & & & & & & & & & & & & & & & & & & & & & \\
\hline
\end{tabular}




\begin{tabular}{|c|c|c|c|c|c|c|c|c|c|c|c|c|c|c|c|c|c|c|c|c|c|c|c|c|}
\hline Entity/Concept & Req & Errors & Count & Flag & 1 & 2 & 3 & 4 & 5 & 6 & 7 & 8 & 9 & $\begin{array}{l}1 \\
0\end{array}$ & $\begin{array}{l}1 \\
1\end{array}$ & $\begin{array}{l}1 \\
2\end{array}$ & $\begin{array}{l}1 \\
3\end{array}$ & $\begin{array}{l}1 \\
4\end{array}$ & $\begin{array}{l}1 \\
5\end{array}$ & & & $\frac{1}{7}$ & $\begin{array}{l}1 \\
8\end{array}$ & $\begin{array}{l}1 \\
9\end{array}$ \\
\hline Type=IfcSlabType; & & & 0 & + & - & - & - & - & - & - & - & - & - & - & - & - & - & - & - & & & - & + & + \\
\hline Material Layer Set Usage & $\mathrm{R}$ & & 0 & + & - & - & - & - & - & - & - & - & - & + & + & + & + & + & + & & & + & + & + \\
\hline Body Geometry & $\mathrm{R}$ & & & & & & & & & & & & & & & & & & & & & & & \\
\hline $\begin{array}{l}\text { RepresentationType=SweptSolid; Ge- } \\
\text { ometry=IfcExtrudedAreaSolid; }\end{array}$ & & & 0 & + & - & - & - & - & - & - & - & - & - & + & + & + & + & + & + & & & + & + & + \\
\hline $\begin{array}{l}\text { RepresentationType=Clipping; Geome- } \\
\text { try=IfcBooleanClippingResult; }\end{array}$ & & & 0 & + & - & - & - & - & - & - & - & - & - & + & + & + & + & + & + & & & + & + & + \\
\hline IfcWall & & & 0 & & & & & & & & & & & & & & & & & & & & & \\
\hline Path Connectivity & $\mathrm{R}$ & & & & & & & & & & & & & & & & & & & & & & & \\
\hline RelatedElement=IfcWall; & & & 0 & + & - & - & - & - & - & - & - & - & - & + & + & + & + & + & + & & & + & + & + \\
\hline Voiding & $\mathrm{R}$ & & & & & & & & & & & & & & & & & & & & & & & \\
\hline RelatedOpening=IfcOpeningElement; & & & 0 & + & - & - & - & - & - & - & - & - & - & + & + & + & + & + & + & & & + & + & + \\
\hline IfcOpeningElement & & & 0 & & & & & & & & & & & & & & & & & & & & & \\
\hline Identity & $\mathrm{R}$ & & 0 & + & - & - & - & - & - & - & - & - & - & + & + & + & + & + & + & & & + & + & + \\
\hline Product Placement & $\mathrm{R}$ & & 0 & + & - & - & - & - & - & - & - & - & - & + & + & + & + & + & + & & & + & + & + \\
\hline Body Geometry & $\mathrm{R}$ & & & & & & & & & & & & & & & & & & & & & & & \\
\hline $\begin{array}{l}\text { RepresentationType=SweptSolid; Ge- } \\
\text { ometry=IfcExtrudedAreaSolid; }\end{array}$ & & & 0 & + & - & - & - & - & - & - & - & - & - & + & + & + & + & + & + & & & + & + & + \\
\hline $\begin{array}{l}\text { RepresentationType=Brep; Geometry= } \\
\text { IfcFacetedBrep; }\end{array}$ & & & 0 & + & - & - & - & - & - & - & - & - & - & + & + & + & + & + & + & & & + & + & + \\
\hline IfcOpeningStandardCase & & & 0 & & & & & & & & & & & & & & & & & & & & & \\
\hline IfcWallStandardCase & & & 0 & & & & & & & & & & & & & & & & & & & & & \\
\hline Identity & $\mathrm{R}$ & & 0 & + & - & - & - & - & - & - & - & - & - & + & + & + & + & + & + & & & + & + & + \\
\hline Object Typing & $\mathrm{O}$ & & & & & & & & & & & & & & & & & & & & & & & \\
\hline Type=lfcWallType; & & & 0 & + & - & - & - & - & - & - & - & - & - & - & - & - & - & - & - & 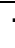 & & - & + & + \\
\hline Material Layer Set Usage & $\mathrm{R}$ & & 0 & + & - & - & - & - & - & - & - & - & - & + & + & + & + & + & + & & & + & + & + \\
\hline Axis Geometry & $\mathrm{R}$ & & & & & & & & & & & & & & & & & & & & & & & \\
\hline $\begin{array}{l}\text { RepresentationType=Curve2D; Geome- } \\
\text { try=IfcBoundedCurve; }\end{array}$ & & & 0 & + & - & - & - & - & - & - & - & - & - & + & + & + & + & + & + & & & + & + & + \\
\hline Body Geometry & $\mathrm{R}$ & & & & & & & & & & & & & & & & & & & & & & & \\
\hline $\begin{array}{l}\text { RepresentationType=SweptSolid; Ge- } \\
\text { ometry=IfcExtrudedAreaSolid; }\end{array}$ & & & 0 & + & - & - & - & - & - & - & - & - & - & + & + & + & + & + & + & & & + & + & + \\
\hline
\end{tabular}




\begin{tabular}{|c|c|c|c|c|c|c|c|c|c|c|c|c|c|c|c|c|c|c|c|c|c|c|c|}
\hline Entity/Concept & Req & Errors & Count & Flag & 1 & 2 & 3 & 4 & 5 & 6 & 7 & 8 & 9 & $\begin{array}{l}1 \\
0\end{array}$ & $\begin{array}{l}1 \\
1\end{array}$ & $\begin{array}{l}1 \\
2\end{array}$ & $\begin{array}{l}1 \\
3\end{array}$ & $\begin{array}{l}1 \\
4\end{array}$ & $\begin{array}{l}1 \\
5\end{array}$ & $\begin{array}{l}1 \\
6\end{array}$ & $\begin{array}{l}1 \\
7\end{array}$ & $\begin{array}{l}\mathbf{1} \\
\mathbf{8}\end{array}$ & $\begin{array}{l}1 \\
9\end{array}$ \\
\hline $\begin{array}{l}\text { RepresentationType=Clipping; Geome- } \\
\text { try=IfcBooleanClippingResult; }\end{array}$ & & & 0 & + & - & - & - & - & - & - & - & - & - & + & + & + & + & + & + & + & + & + & + \\
\hline IfcSlab & & & 0 & & & & & & & & & & & & & & & & & & & & \\
\hline Voiding & $\mathrm{R}$ & & 0 & + & - & - & - & - & - & - & - & - & - & + & + & + & + & + & + & + & + & + & + \\
\hline SUMMARY FOR IFC4 & & & & & $\begin{array}{r}99 \\
\% \\
\end{array}$ & $\begin{array}{r}99 \\
\% \\
\end{array}$ & $\begin{array}{r}99 \\
\%\end{array}$ & $\begin{array}{r}99 \\
\%\end{array}$ & $\begin{array}{r}99 \\
\%\end{array}$ & $\begin{array}{r}99 \\
\%\end{array}$ & $\begin{array}{r}99 \\
\%\end{array}$ & $\begin{array}{r}99 \\
\%\end{array}$ & $\begin{array}{r}99 \\
\%\end{array}$ & $\begin{array}{r}9 \\
9 \\
\%\end{array}$ & $\begin{array}{r}9 \\
9 \\
\%\end{array}$ & $\begin{array}{r}9 \\
9 \\
\%\end{array}$ & $\begin{array}{c}9 \\
8 \\
\%\end{array}$ & $\begin{array}{r}9 \\
9 \\
\%\end{array}$ & $\begin{array}{r}9 \\
9 \\
\%\end{array}$ & $\begin{array}{r}9 \\
9 \\
\%\end{array}$ & $\begin{array}{r}9 \\
9 \\
\%\end{array}$ & $\begin{array}{r}9 \\
6 \\
\%\end{array}$ & $\begin{array}{r}9 \\
6 \\
\%\end{array}$ \\
\hline SUMMARY FOR IFC2X3 & & & & & $\begin{array}{r}99 \\
\%\end{array}$ & $\begin{array}{r}99 \\
\%\end{array}$ & $\begin{array}{r}99 \\
\%\end{array}$ & $\begin{array}{r}99 \\
\%\end{array}$ & $\begin{array}{r}99 \\
\%\end{array}$ & $\begin{array}{r}99 \\
\%\end{array}$ & $\begin{array}{r}10 \\
0 \\
\%\end{array}$ & $\begin{array}{r}99 \\
\%\end{array}$ & $\begin{array}{r}10 \\
0 \\
\%\end{array}$ & $\begin{array}{r}9 \\
9 \\
\%\end{array}$ & $\begin{array}{r}9 \\
9 \\
\%\end{array}$ & $\begin{array}{r}9 \\
9 \\
\%\end{array}$ & $\begin{array}{r}9 \\
9 \\
\% \\
\end{array}$ & $\begin{array}{r}9 \\
9 \\
\%\end{array}$ & $\begin{array}{r}9 \\
9 \\
\%\end{array}$ & $\begin{array}{r}9 \\
9 \\
\%\end{array}$ & $\begin{array}{r}9 \\
9 \\
\%\end{array}$ & $\begin{array}{r}9 \\
8 \\
\%\end{array}$ & $\begin{array}{r}9 \\
7 \\
\%\end{array}$ \\
\hline
\end{tabular}


Table C4. Summary of water system MVD IFC validation.

\begin{tabular}{|c|c|c|c|c|c|c|c|c|c|c|c|c|c|c|c|c|c|c|c|c|}
\hline & & \multicolumn{19}{|c|}{ Exchange } \\
\hline & Flag & 1 & 2 & 3 & 4 & 5 & 6 & 7 & 8 & 9 & 10 & 11 & 12 & 13 & 14 & 15 & 16 & 17 & 18 & 19 \\
\hline $\begin{array}{l}\text { Test was inappli- } \\
\text { cable for the par- } \\
\text { ticular exchange (-) }\end{array}$ & 11 & 372 & 368 & 323 & 264 & 264 & 258 & 253 & 258 & 251 & 161 & 161 & 154 & 154 & 154 & 131 & 120 & 120 & 59 & 52 \\
\hline $\begin{array}{l}\text { Entity or concept } \\
\text { passed the test for } \\
\text { the given ex- } \\
\text { change }(+)\end{array}$ & 368 & 25 & 30 & 69 & 129 & 129 & 135 & 140 & 135 & 142 & 230 & 230 & 236 & 236 & 236 & 259 & 270 & 270 & 322 & 329 \\
\hline $\begin{array}{l}\text { Optional rule for } \\
\text { the particular ex- } \\
\text { change failed the } \\
\text { test }\left(^{*}\right)\end{array}$ & 22 & 19 & 18 & 24 & 23 & 23 & 23 & 24 & 23 & 24 & 24 & 24 & 24 & 22 & 24 & 24 & 24 & 24 & 24 & 21 \\
\hline $\begin{array}{l}\text { Mandatory rule for } \\
\text { the particular ex- } \\
\text { change failed the } \\
\text { test }(F)\end{array}$ & 18 & 3 & 3 & 3 & 3 & 3 & 3 & 2 & 3 & 2 & 4 & 4 & 5 & 7 & 5 & 5 & 5 & 5 & 14 & 17 \\
\hline Total Tests & 419 & 419 & 419 & 419 & 419 & 419 & 419 & 419 & 419 & 419 & 419 & 419 & 419 & 419 & 419 & 419 & 419 & 419 & 419 & 419 \\
\hline \multicolumn{21}{|l|}{ Office Building } \\
\hline & & \multicolumn{19}{|c|}{ Exchange } \\
\hline & Flag & 1 & 2 & 3 & 4 & 5 & 6 & 7 & 8 & 9 & 10 & 11 & 12 & 13 & 14 & 15 & 16 & 17 & 18 & 19 \\
\hline $\begin{array}{l}\text { Test was inappli- } \\
\text { cable for the par- } \\
\text { ticular exchange (-) }\end{array}$ & 11 & 372 & 368 & 323 & 264 & 264 & 258 & 253 & 258 & 251 & 161 & 161 & 154 & 154 & 154 & 131 & 120 & 120 & 59 & 52 \\
\hline $\begin{array}{l}\text { Entity or concept } \\
\text { passed the test for } \\
\text { the given ex- } \\
\text { change }(+)\end{array}$ & 368 & 25 & 30 & 69 & 129 & 129 & 135 & 140 & 135 & 142 & 230 & 230 & 236 & 236 & 236 & 259 & 270 & 270 & 322 & 329 \\
\hline $\begin{array}{l}\text { Optional rule for } \\
\text { the particular ex- } \\
\text { change failed the } \\
\text { test }\left(^{\star}\right)\end{array}$ & 22 & 19 & 18 & 24 & 23 & 23 & 23 & 24 & 23 & 24 & 24 & 24 & 24 & 22 & 24 & 24 & 24 & 24 & 24 & 21 \\
\hline $\begin{array}{l}\text { Mandatory rule for } \\
\text { the particular ex- } \\
\text { change failed the } \\
\text { test }(F)\end{array}$ & 18 & 3 & 3 & 3 & 3 & 3 & 3 & 2 & 3 & 2 & 4 & 4 & 5 & 7 & 5 & 5 & 5 & 5 & 14 & 17 \\
\hline Total Tests & 419 & 419 & 419 & 419 & 419 & 419 & 419 & 419 & 419 & 419 & 419 & 419 & 419 & 419 & 419 & 419 & 419 & 419 & 419 & 419 \\
\hline \multicolumn{21}{|l|}{ Medical Clinic } \\
\hline & & \multicolumn{19}{|c|}{ Exchange } \\
\hline & Flag & 1 & 2 & 3 & 4 & 5 & 6 & 7 & 8 & 9 & 10 & 11 & 12 & 13 & 14 & 15 & 16 & 17 & 18 & 19 \\
\hline $\begin{array}{l}\text { Test was inappli- } \\
\text { cable for the par- } \\
\text { ticular exchange (-) }\end{array}$ & 11 & 372 & 368 & 323 & 264 & 264 & 258 & 253 & 258 & 251 & 161 & 161 & 154 & 154 & 154 & 131 & 120 & 120 & 59 & 52 \\
\hline $\begin{array}{l}\text { Entity or concept } \\
\text { passed the test for } \\
\text { the given ex- } \\
\text { change }(+)\end{array}$ & 373 & 26 & 31 & 73 & 133 & 133 & 139 & 144 & 139 & 146 & 234 & 234 & 241 & 241 & 241 & 264 & 275 & 275 & 327 & 334 \\
\hline $\begin{array}{l}\text { Optional rule for } \\
\text { the particular ex- } \\
\text { change failed the } \\
\text { test }\left(^{*}\right)\end{array}$ & 18 & 19 & 18 & 21 & 20 & 20 & 20 & 21 & 20 & 21 & 21 & 21 & 20 & 18 & 20 & 20 & 20 & 20 & 20 & 17 \\
\hline $\begin{array}{l}\text { Mandatory rule for } \\
\text { the particular ex- } \\
\text { change failed the } \\
\text { test }(F)\end{array}$ & 17 & 2 & 2 & 2 & 2 & 2 & 2 & 1 & 2 & 1 & 3 & 3 & 4 & 6 & 4 & 4 & 4 & 4 & 13 & 16 \\
\hline Total Tests & 419 & 419 & 419 & 419 & 419 & 419 & 419 & 419 & 419 & 419 & 419 & 419 & 419 & 419 & 419 & 419 & 419 & 419 & 419 & 419 \\
\hline
\end{tabular}




\section{REPORT DOCUMENTATION PAGE}

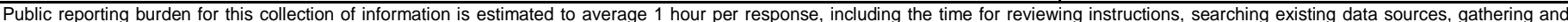

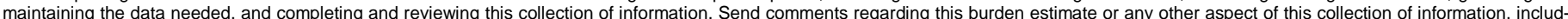

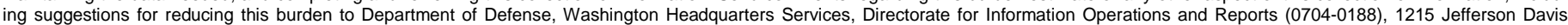

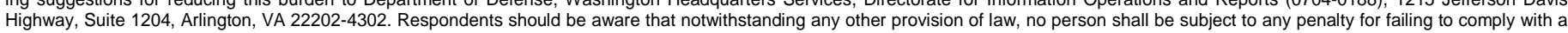
collection of information if it does not display a currently valid OMB control number. PLEASE DO NOT RETURN YOUR FORM TO THE ABOVE ADDRESS.

\begin{tabular}{l|l|l|} 
1. REPORT DATE (DD-MM-YYYY) & 2. REPORT TYPE & 3. DATES COVERED (FrOm - To)
\end{tabular}

June 2013 Final

4. TITLE AND SUBTITLE

Ontology for Life-Cycle Modeling of Water Distribution Systems: Application of

Model View Definition Attributes

5a. CONTRACT NUMBER

CRADA-07-CERL-02

5b. GRANT NUMBER

5c. PROGRAM ELEMENT NUMBER

622784 T41

6. AUTHOR(S)

Kristine K. Fallon, Robert A. Feldman, Gregory Williams, and Omobolawa

Fadojutimi, and Tim Chipman

5d. PROJECT NUMBER

5e. TASK NUMBER

5f. WORK UNIT NUMBER

7. PERFORMING ORGANIZATION NAME(S) AND ADDRESS(ES)

Kristine Fallon Associates, Inc.

8. PERFORMING ORGANIZATION REPORT NUMBER

Chicago, IL 60603

9. SPONSORING I MONITORING AGENCY NAME(S) AND ADDRESS(ES)

US Army Engineer Research and Development Center

Construction Engineering Research Laboratory

P.O. Box 9005

Champaign, IL 61826-9005

10. SPONSOR/MONITOR'S ACRONYM(S)

ERDC-CERL

11. SPONSOR/MONITOR'S REPORT NUMBER(S)

ERDC/CERL CR-13-5

\section{DISTRIBUTION / AVAILABILITY STATEMENT}

Approved for public release; distribution is unlimited.

\section{SUPPLEMENTARY NOTES}

\section{ABSTRACT}

Previous efforts by the US Army Engineer Research and Development Center, Construction Engineering Research Laboratory (ERDC-CERL) to develop a life-cycle building model have resulted in the definition of a "core” building information model that contains general information describing facility assets such as spaces and equipment. To describe how facility assets (i.e., components) function together, information about assemblies of assets and their connections must also be defined. The definitions of assets, assemblies, and connections for the various building-information domains are discipline-specific. Work documented in ERDC/CERL CR-13-4 identified the processes and tasks specifically associated with the design of interior plumbing systems and the information exchange requirements for every participant in the design. The findings were used to develop an information-exchange Model View Definition (MVD) for building water systems.

The objective of the current work was to document the steps needed to identify the plumbing MVD attributes in three experimental building information models representing typical low-rise Army facilities, and to update those models. This work also validated the International Foundation Class (IFC) export function from the experimental models against the water system MVD, and studied the requirements for creating computable open building models that can be utilized for the automated information exchanges.

\section{SUBJECT TERMS}

information exchange, interior plumbing systems, product data templates, guide specifications, Construction Operations Building information exchange (COBie), Building Information Modeling (BIM)

\begin{tabular}{l|l} 
16. SECURITY CLASSIFICATION OF: & 17. LIMITATION
\end{tabular}

\begin{tabular}{l|l} 
17. SECURITY CLASSIFICATION OF: & OF ABSTRACT
\end{tabular}

\begin{tabular}{|l|l|r|}
\hline $\begin{array}{c}\text { a. REPORT } \\
\text { Unclassified }\end{array}$ & b. ABSTRACT & c. THIS PAGE \\
Unclassified & Unclassified
\end{tabular}

\begin{tabular}{l|} 
18. NUMBER \\
OF PAGES \\
\cline { 2 - 2 }
\end{tabular}

\section{9a. NAME OF RESPONSIBLE} PERSON

19b. TELEPHONE NUMBER (include area code) 